Supporting Information for

\title{
Nature of Oxygen Adsorption on Defective Carbonaceous Materials
}

\author{
Zongtang Fang, ${ }^{a^{*}}$ Lan Li, ${ }^{b, c}$ David A. Dixon, ${ }^{\mathrm{d}}$ Rebecca R. Fushimi, ${ }^{\text {a }}$ Eric J. Dufek, ${ }^{\text {e* }}$ \\ ${ }^{a}$ Biological and Chemical Science and Engineering Department, Idaho National Laboratory, \\ Idaho Falls, ID 83415 \\ ${ }^{\mathrm{b}}$ Micron School of Materials Science and Engineering, Boise State University, Boise, ID 83725 \\ ${ }^{\mathrm{c}}$ Center for Advanced Energy Studies, Idaho Falls, ID 83401 \\ d Department of Chemistry and Biochemistry, The University of Alabama, Shelby Hall, \\ Tuscaloosa AL 35487 \\ ${ }^{\mathrm{e}}$ Energy Storage and Advanced Transportation Department, Idaho National Laboratory, Idaho \\ Falls, ID 83415
}

Complete Ref. 58

Frisch, M. J.; Trucks, G. W.; Schlegel, H. B.; Scuseria, G. E.; Robb, M. A.; Cheeseman, J. R.; Scalmani, G.; Barone, V.; Petersson, G. A.; Nakatsuji, H.; Li, X.; Caricato, M.; Marenich, A. V.; Bloino, J.; Janesko, B. G.; Gomperts, R.; Mennucci, B.; Hratchian, H. P.; Ortiz, J. V.; Izmaylov, A. F.; Sonnenberg, J. L.; Williams-Young, D.; Ding, F.; Lipparini, F.; Egidi, F.; Goings, J.; Peng, B.; Petrone, A.; Henderson, T.; Ranasinghe, D.; Zakrzewski, V. G.; Gao, J.; Rega, N.; Zheng, G.; Liang, W.; Hada, M.; Ehara, M.; Toyota, K.; Fukuda, R.; Hasegawa, J.; Ishida, M.; Nakajima, T.; Honda, Y.; Kitao, O.; Nakai, H.; Vreven, T.; Throssell, K.; Montgomery, J. A., Jr.; Peralta, J. E.; Ogliaro, F.; Bearpark, M. J.; Heyd, J. J.; Brothers, E. N.; Kudin, K. N.; Staroverov, V. N.; Keith, T. A.; Kobayashi, R.; Normand, J.; Raghavachari, K.; Rendell, A. P.; Burant, J. C.; Iyengar, S. S.; Tomasi, J.; Cossi, M.; Millam, J. M.; Klene, M.; Adamo, C.; Cammi, R.; Ochterski, J. W.; Martin, R. L.; Morokuma, K.; Farkas, O.; Foresman,J. B.; Fox, D. J. Gaussian 16, Revision A.03, Gaussian, Inc., Wallingford CT, 2016.

*Correspondence authors: zongtang.fang@inl.gov; eric.dufek@inl.gov 


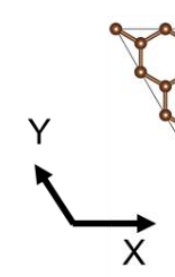

Graphene

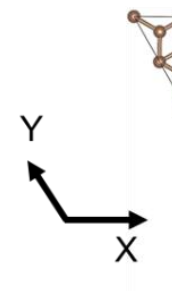

Graphite

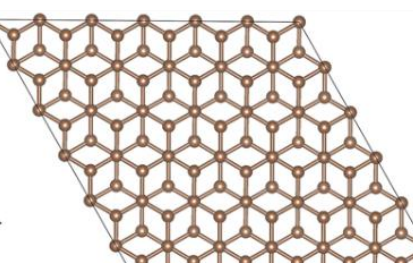
ialaiaia.

Defect free

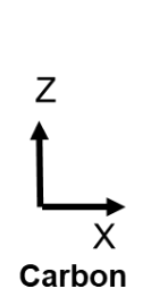

Carbon

\section{Defect Free}

Defect free

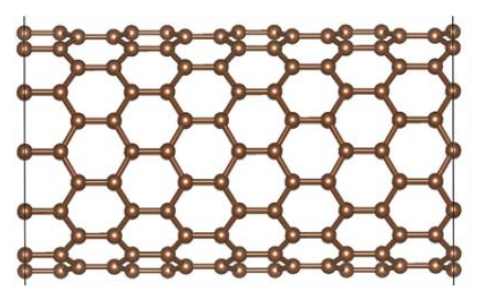

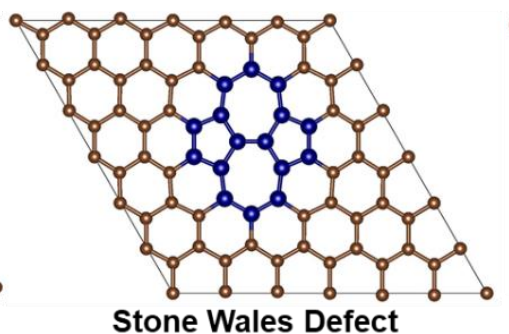

Stone Wales Defect

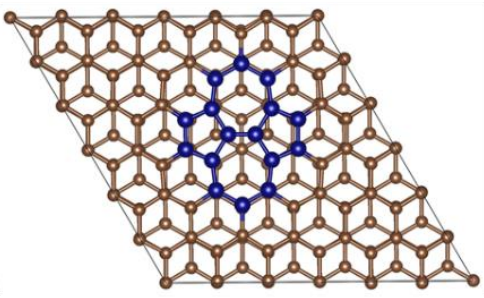

Stone Wales Defect

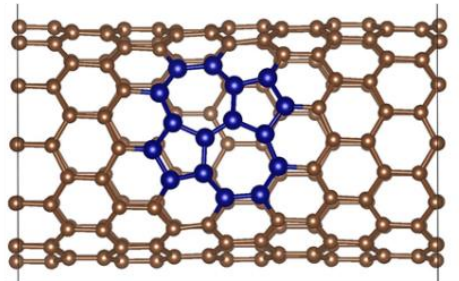

Stone Wales Defect
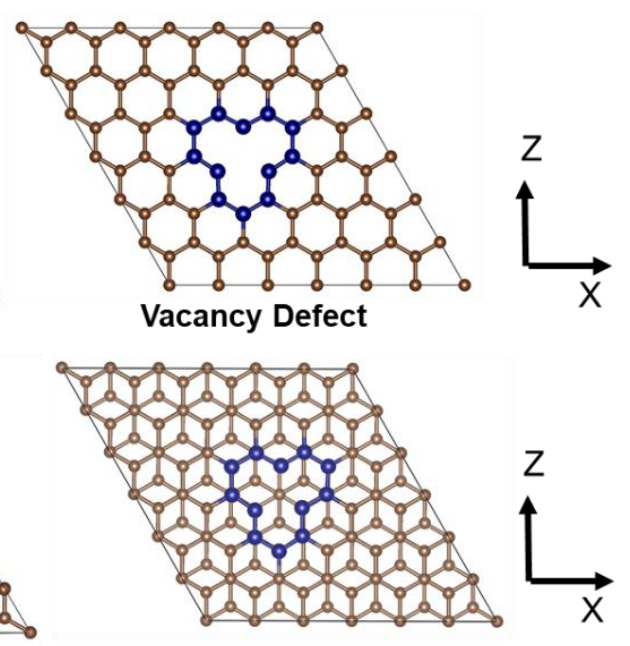

Vacancy Defect

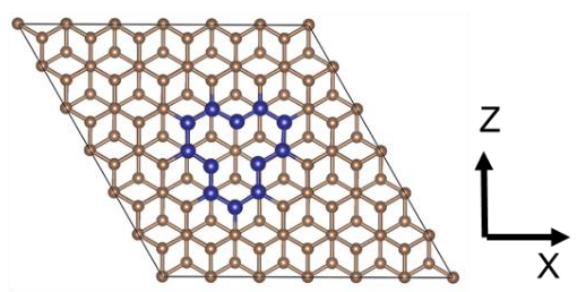

Vacancy Defect

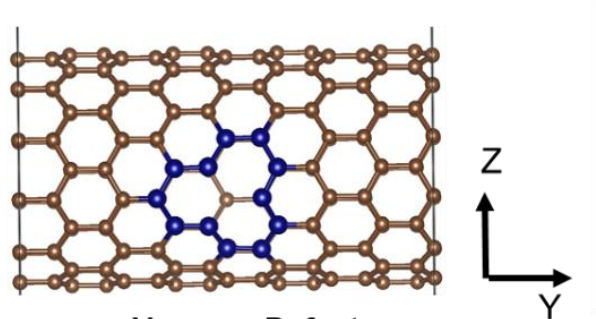

Vacancy Defect
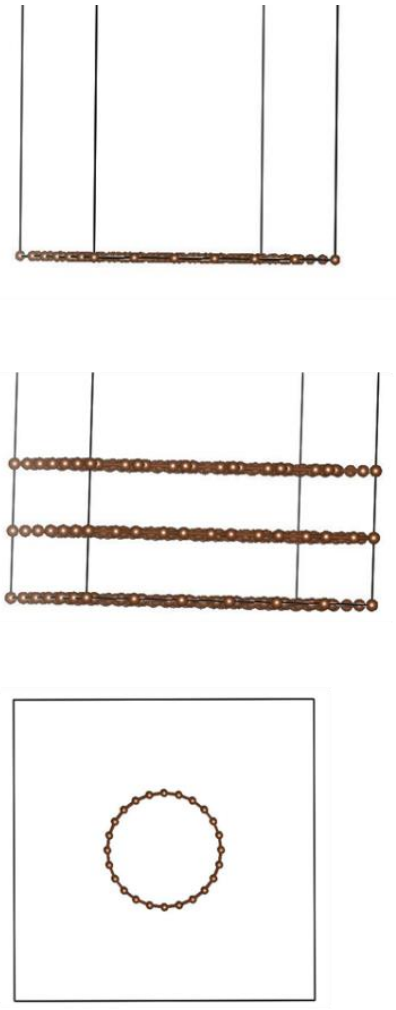

Figure S1 Top views of the basal planes of defect free, Stone-Wales (SW) defected, single vacancy defected graphene and graphite; Side views of defect free, SW defected, single vacancy defected $(12,0)$ zigzag single-walled carbon nanotubes. 


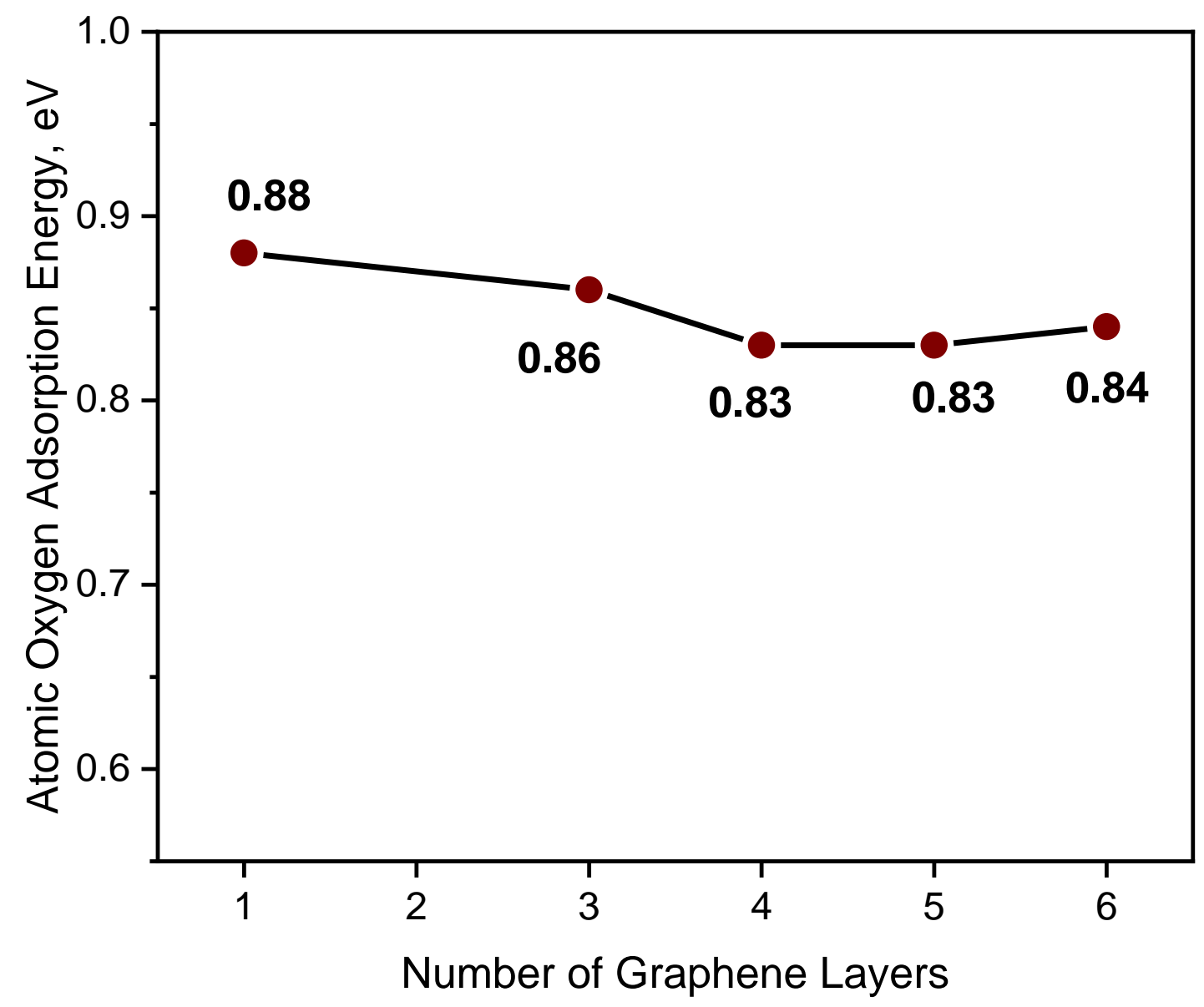

Figure S2. Adsorption energies in $\mathrm{eV}$ for atomic oxygen adsorption on different graphene layers with optPBE-vdw. The adsorption energy is predicted to be $0.94 \mathrm{eV}$ with $\mathrm{PBE}$ on one-layer graphene. 


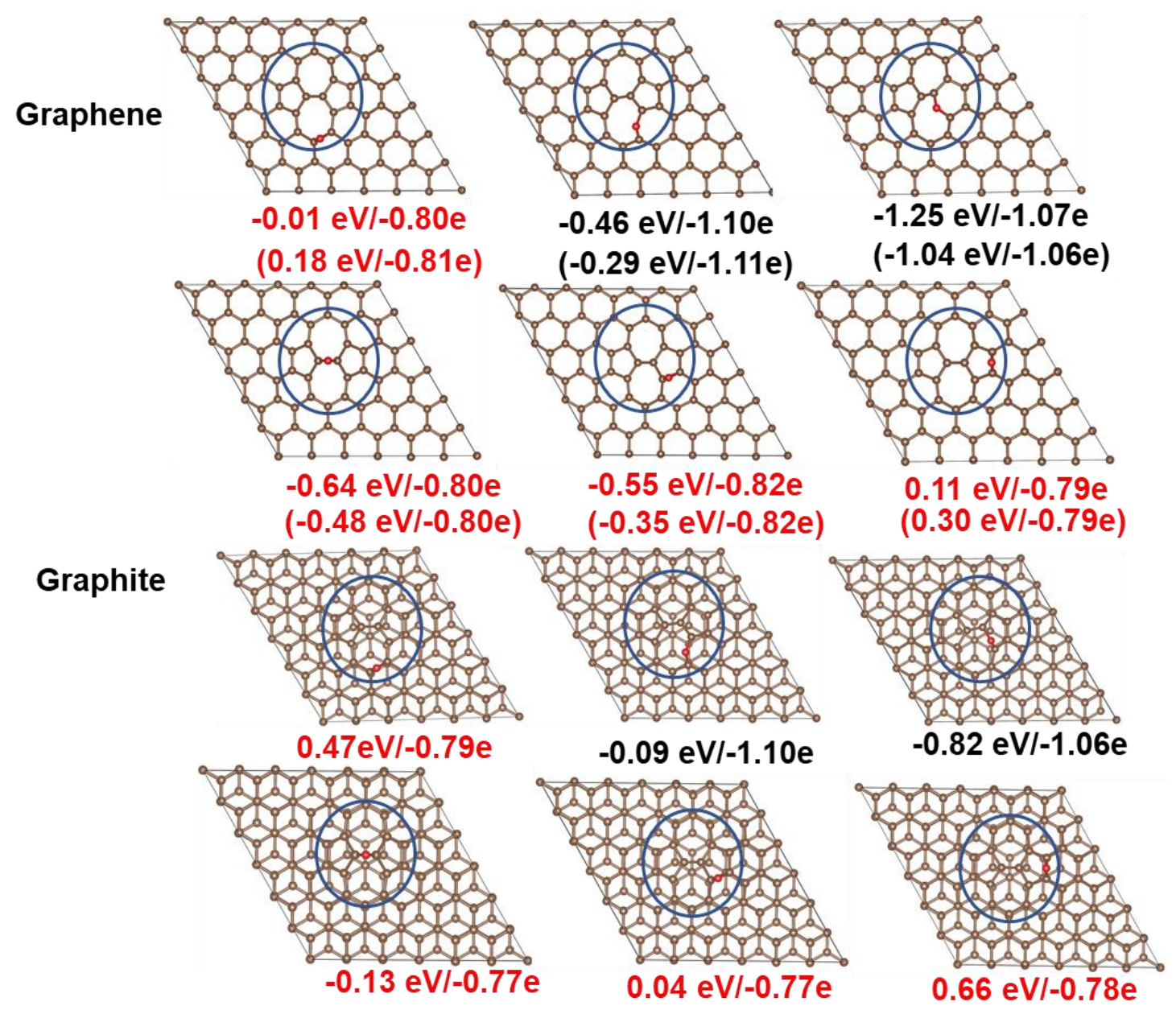

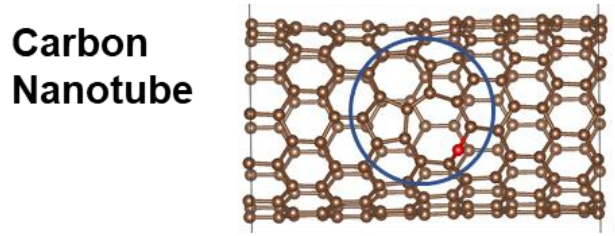

$0.12 \mathrm{eV} /-1.09 \mathrm{e}$

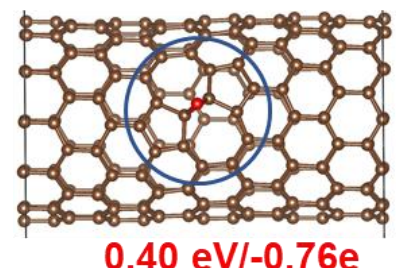

$0.40 \mathrm{eV} /-0.76 \mathrm{e}$

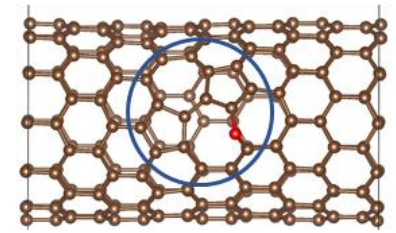

$-0.44 \mathrm{eV} / \mathrm{-1.09e}$

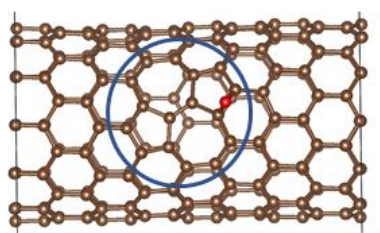

$0.02 \mathrm{eV} /-0.81 \mathrm{e}$
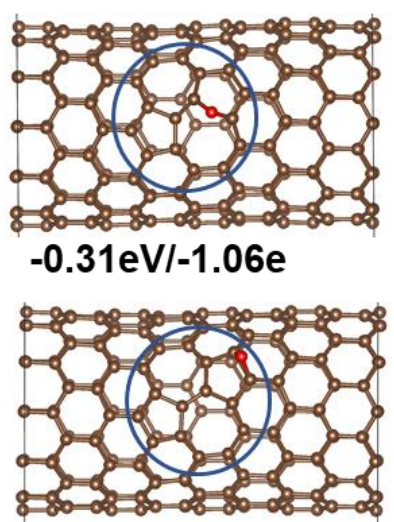

$0.45 \mathrm{eV} /-0.88 \mathrm{e}$

Figure S3. The adsorption energies in $\mathrm{eV}$ as well as the net Bader charge of $\mathrm{O}$ on various adsorption sites around a SW defect on studied graphene, graphite, and carbon nanotube. The energies are predicted at the optPBE-vdw level for graphene and graphite and with PBE for nanotube; The PBE values for graphene are shown in parenthesis. The energies and charges in black and in red are for the adsorption leading to the formation of an ether and an epoxide respectively. 


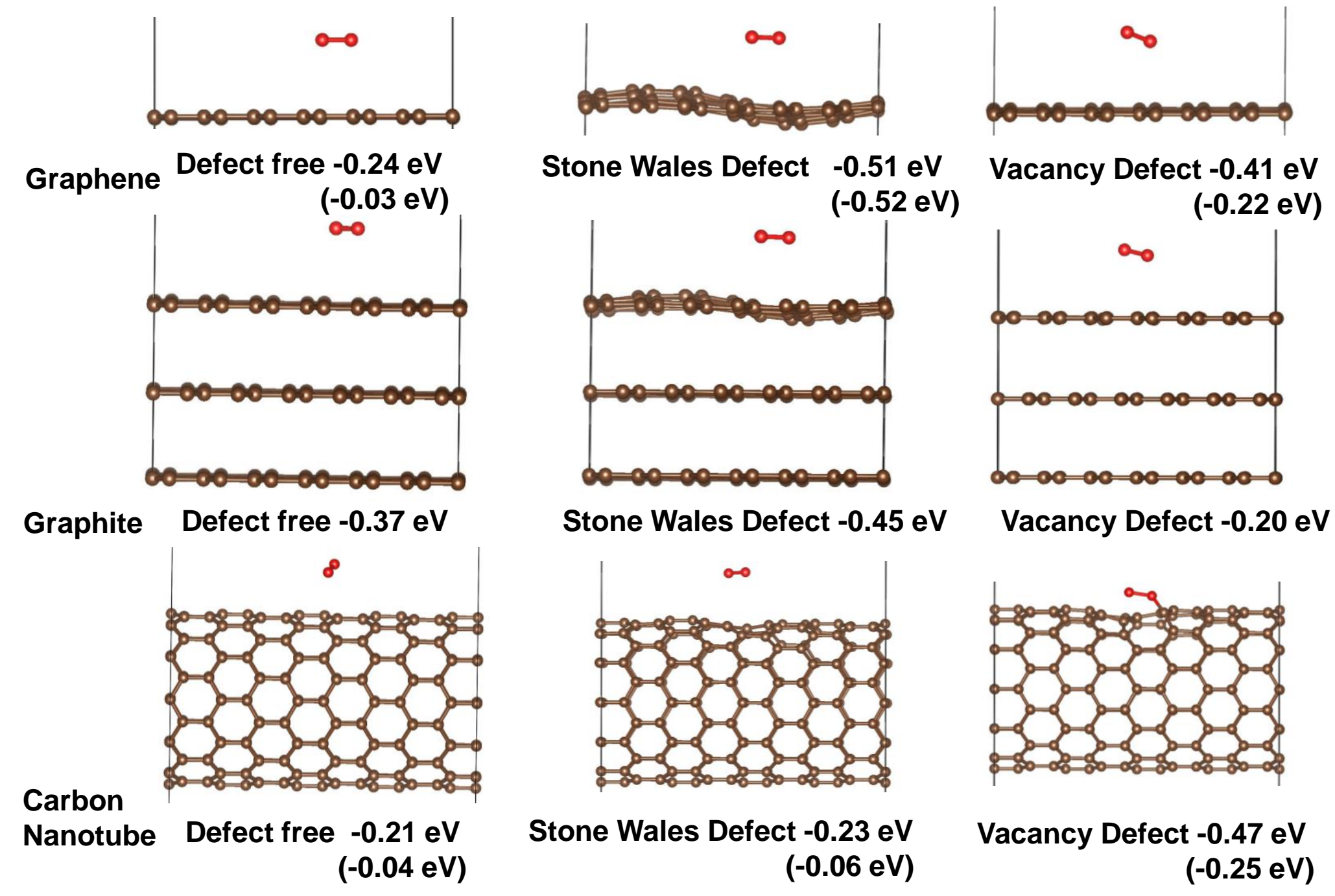

Figure S4. Side views of molecular oxygen adsorption on defect free, SW defected, and SV defected graphene, graphite, and carbon nanotubes and the adsorption energies in $\mathrm{eV}$. The energies are predicted at the optPBE-vdw level; The PBE values for graphene and nanotubes are shown in parenthesis. 


\section{Graphene}

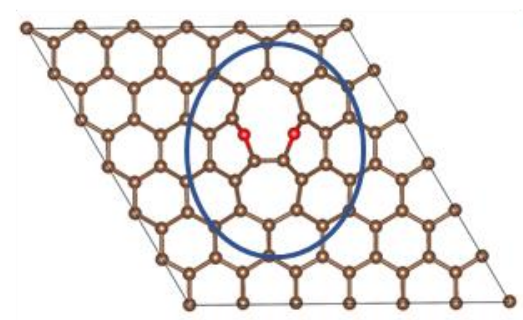

$-1.38 \mathrm{eV}$

$(-1.08 \mathrm{eV})$

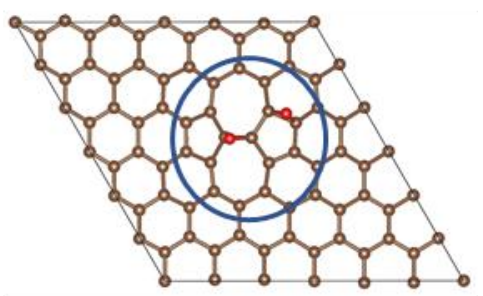

$-0.61 \mathrm{eV}$

$(-0.36 \mathrm{eV})$

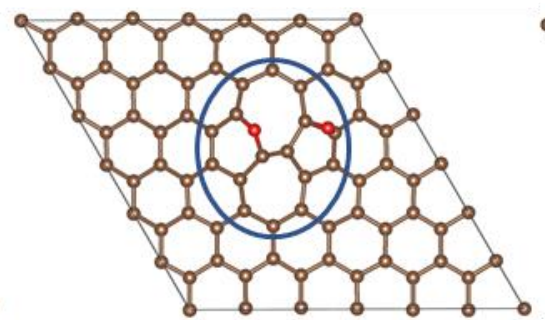

$-0.57 \mathrm{eV}$

$(-0.26 \mathrm{eV})$

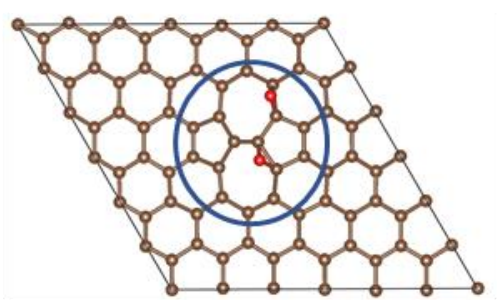

$-0.50 \mathrm{eV}$

$(-0.27 \mathrm{eV})$

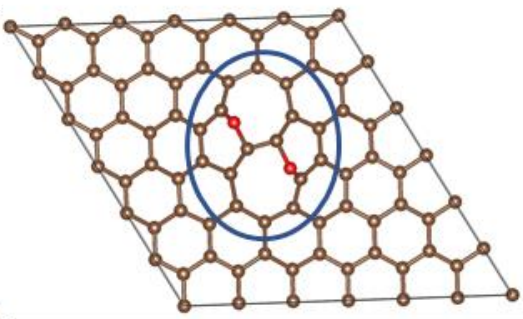

$0.44 \mathrm{eV}$

(0.76 eV)

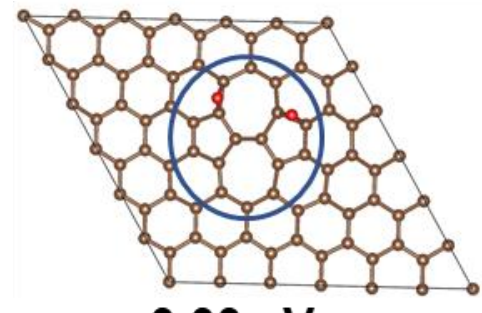

$-0.03 \mathrm{eV}$

(0.20 eV)

\section{Graphite}

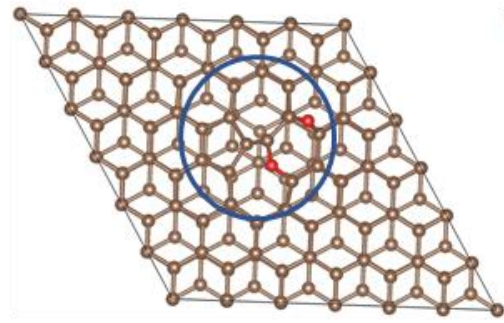

$-0.71 \mathrm{eV}$

Carbon Nanotube

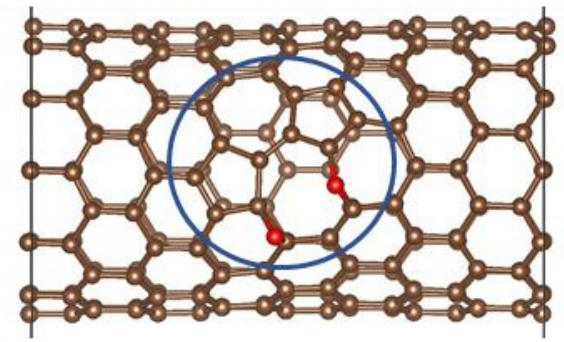

$-0.25 \mathrm{eV}$

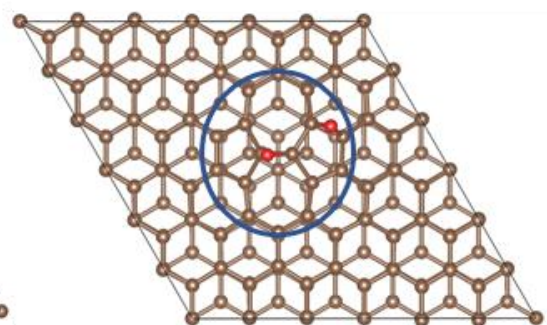

$-0.01 \mathrm{eV}$

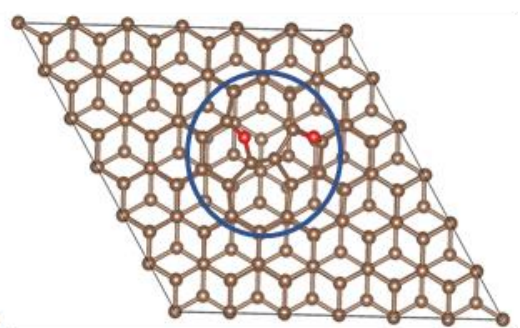

$0.00 \mathrm{eV}$

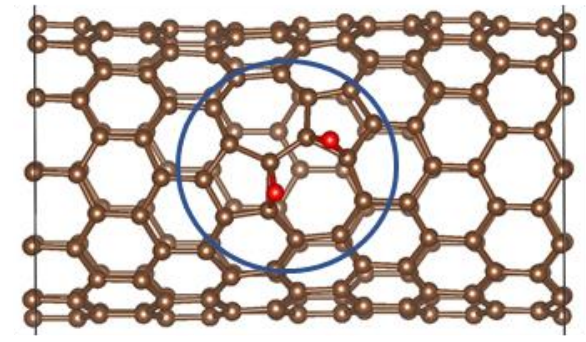

$1.24 \mathrm{eV}$

Figure S5. Additional structures and energetics for the chemisorption of $\mathrm{O}_{2}$ on different binding site for graphene, graphite and carbon nanotube. The energies are predicted at the optPBE-vdw level for graphene and graphite and with PBE for nanotube; The PBE values for graphene are shown in parenthesis. 


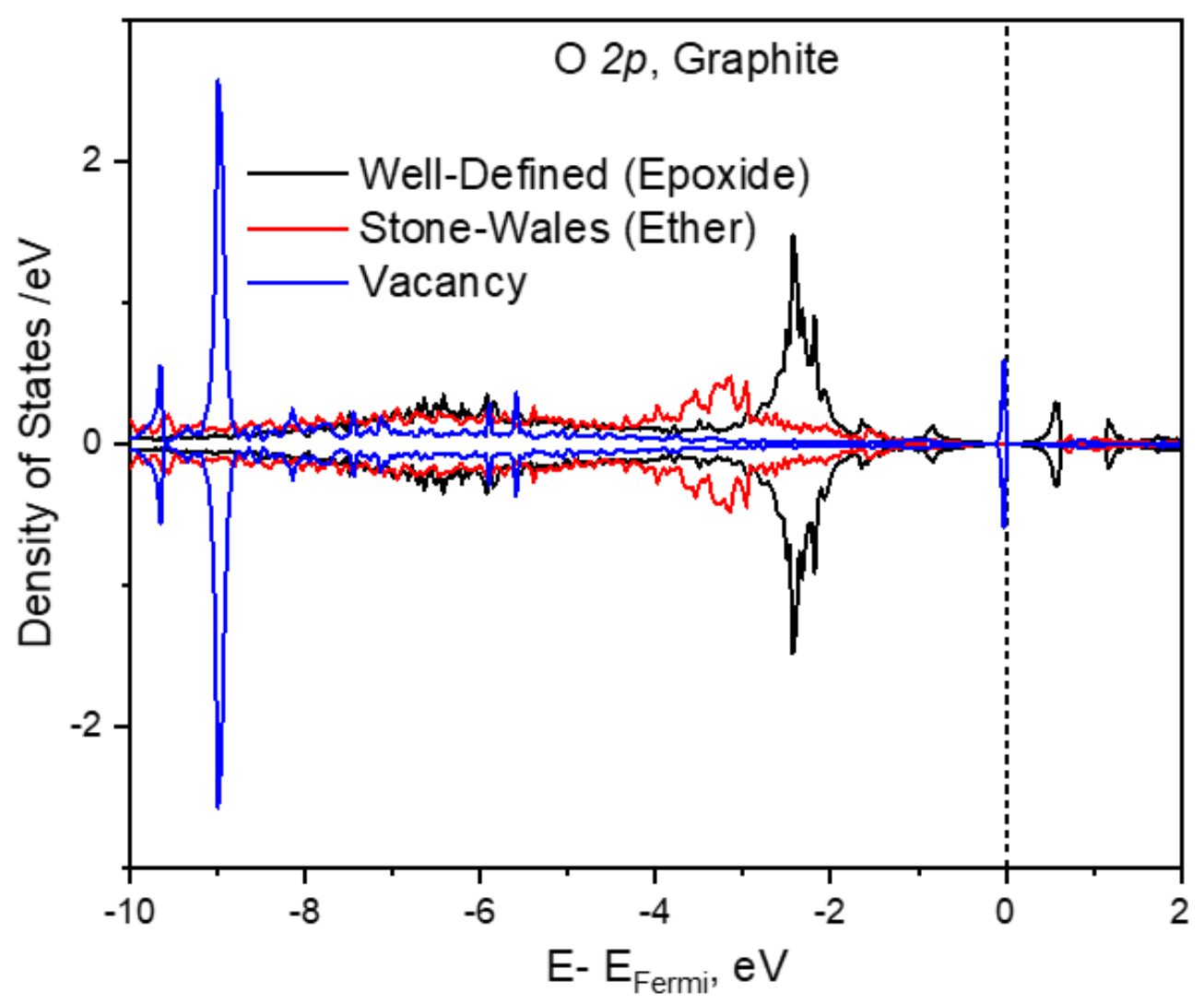

Figure S6. Projected density of states of $\mathrm{O} 2 p$ orbitals in the products of atomic adsorption on defect free, SW defected, and single vacancy defected graphite at the optPBE-vdw level. The structures correspond to Figures $1 \mathrm{~d}$ and $1 \mathrm{f}$ in the manuscript. 


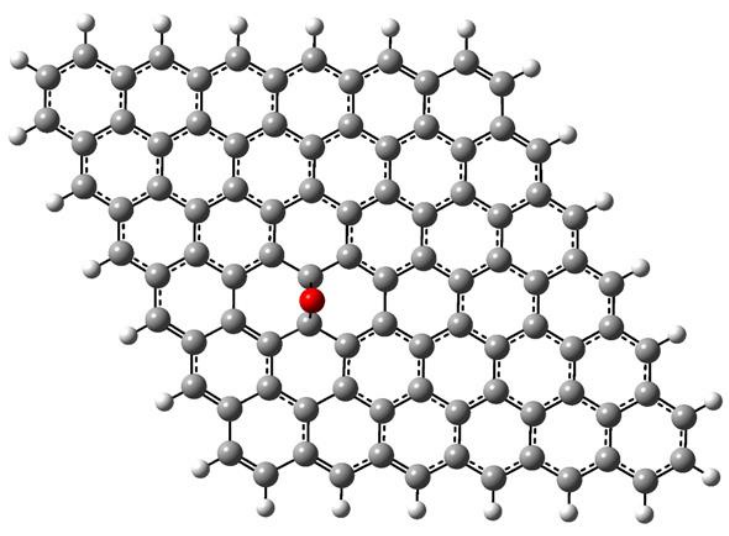

(a) Epoxide

Triplet 0.28

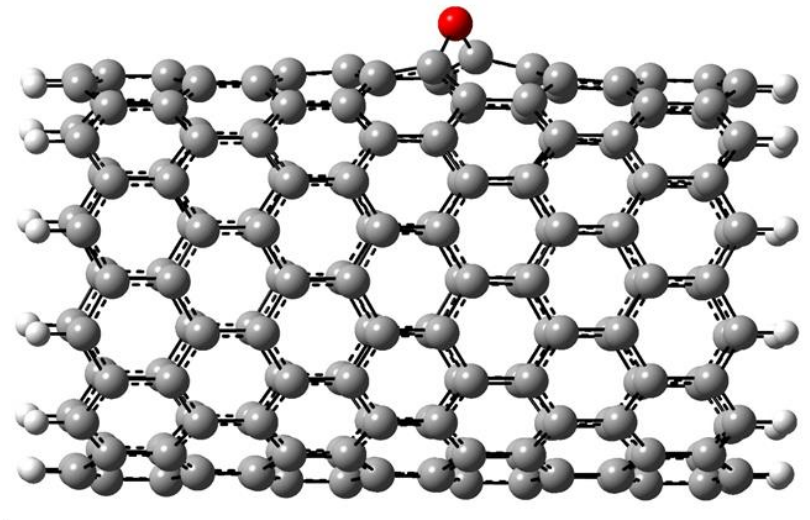

(b) Ether

\section{Triplet -2.60}

Figure 7. Optimized molecular structures and atomic oxygen adsorption energies $\left(\Delta \mathrm{H}_{0 \mathrm{~K}}\right)$ in $\mathrm{eV}$ for graphene (a) and nanotube (b) with cluster models at the M06-2X/6-311G(d,p) level using a larger model. For the graphene, there are 96 carbon atoms; For the nanotube, there are 192 carbon atoms. One half of the electronic energy of $\mathrm{O}_{2}$ was selected for the adsorbate. $\mathrm{T}=$ Triplet in black. 
Table S1. Calculated bond distances in $\AA$ and bond angles in degree for atomic oxygen adsorption with optPBE-vdw for graphene and graphite and with PBE for nanotubes.

\begin{tabular}{|c|c|c|c|c|c|}
\hline \multicolumn{2}{|r|}{ Material } & $\mathrm{C} 1-\mathrm{O}$ & $\mathrm{C} 2-\mathrm{O}$ & $\mathrm{C} 3-\mathrm{O}$ & $\angle \mathrm{C}-\mathrm{O}-\mathrm{C}$ \\
\hline \multirow{3}{*}{ Graphene } & Defect free & 1.464 & 1.465 & & 62.0 \\
\hline & Stone-Wales Defect & 1.382 & 1.395 & & 102.9 \\
\hline & Single vacancy ${ }^{\mathrm{a}}$ & 1.478 & 1.478 & 1.478 & 120.0 \\
\hline \multirow{3}{*}{ Graphite } & Defect free & 1.485 & 1.486 & & 60.7 \\
\hline & Stone-Wales Defect & 1.389 & 1.403 & & 102.5 \\
\hline & Single vacancy ${ }^{\mathrm{a}}$ & 1.488 & 1.488 & 1.488 & 120.0 \\
\hline \multirow{3}{*}{ Nanotube } & Defect free & 1.390 & 1.390 & & 95.3 \\
\hline & Stone-Wales Defect & 1.384 & 1.403 & & 102.3 \\
\hline & Single vacancy & 1.492 & 1.518 & 1.518 & $112.7,116.6,116.6$ \\
\hline
\end{tabular}

${ }^{a}$ The three $\angle \mathrm{C}-\mathrm{O}-\mathrm{C}$ are identical with $120^{\circ}$. 
Table S2. Detailed hybridization properties on the alpha and beta spin orbitals to make the C-O bonds in the epoxide and the ether on graphene and nanotube from NBO analysis calculated at the M06-2X/6-311G(d,p) level.

\begin{tabular}{|c|c|c|c|c|c|c|c|}
\hline $\mathrm{S}$ & ond & $\begin{array}{l}\text { Hybrid }^{\mathrm{a}} \\
h_{\mathrm{C}}(\alpha, \beta)\end{array}$ & $\begin{array}{l}\% p^{b} \\
(\alpha, \beta)\end{array}$ & $\begin{array}{l}\text { Hybrid }^{\mathrm{a}} \\
h_{\mathrm{O}}(\alpha, \beta)\end{array}$ & $\begin{array}{l}\% p^{b} \\
(\alpha, \beta)\end{array}$ & & \\
\hline \multirow{2}{*}{$\begin{array}{l}\text { Graphene- } \\
\text { Epoxide }\end{array}$} & & & $6.0 \%, 86$ & 607867 & $.4 \%, 87$ & $5.6 \%, 35$. & $.4 \%, 65.0 \%)$ \\
\hline & & & $360 \%$ & & ) & 35. & \%) \\
\hline \multirow{2}{*}{$\begin{array}{l}\text { Graphene } \\
\text { SW- Ether }\end{array}$} & & & $(77.1 \%, 77$ & $\left.\mathrm{sp}^{2.85}, \mathrm{sp}^{2.86}\right)$ & 40 & 32.8 & $2 \%)$ \\
\hline & $-\mathrm{O}$ & $\left.7, \mathrm{sp}^{3.48}\right)$ & $(77.6 \%, 77$ & $\left(\mathrm{sp}^{2.97}, \mathrm{sp}^{3.00}\right)$ & $(74.8 \%, 7$ & 6) & $7.0 \%)$ \\
\hline \multirow{2}{*}{$\begin{array}{l}\text { Nanotube } \\
\text { Ether }\end{array}$} & & & $(76.6 \%, 76.6$ & $\left.\mathrm{sp}^{3.06}, \mathrm{sp}^{3.10}\right)$ & $754 \% 7$ & $33.5 \%$ & $\left(66.5^{\circ}\right.$ \\
\hline & & $\left.\mathrm{sp}^{3.28}\right)$ & $(76.7 \%, 7$ & $\left(\mathrm{sp}^{3.11}, \mathrm{sp}\right.$ & $(76.7 \%, 75.4 \%)$ & $33.5 \%)$ & $(66.6 \%, 66.5 \%)$ \\
\hline \multirow{2}{*}{$\begin{array}{l}\text { Nanotube } \\
\text { SW-Ether }\end{array}$} & & & $(77.9 \%, 77.8 \%)$ & & & 12207 & $(66.8 \%, 66.9 \%)$ \\
\hline & $\mathrm{C} 2-\mathrm{O}$ & $\left.\mathrm{sp}^{3.37}, \mathrm{sp}^{3.36}\right)$ & $(77.1 \%, 77.1 \%)$ & $\left(\mathrm{sp}^{2.79}, \mathrm{sp}^{2.77}\right)$ & $(73.6 \%, 73.4 \%)$ & $(33.0 \%, 33.0 \%)$ & $(67.0 \%, 67.0 \%$ \\
\hline
\end{tabular}

${ }^{\mathrm{a}}$ Composition of the natural hybrids of $\mathrm{C}$ and $\mathrm{O} ;{ }^{\mathrm{b}}$ Percentage of $p$ character of the natural hybrids of $\mathrm{C}$ and $\mathrm{O} ;{ }^{\mathrm{c}} \mathrm{Weights}$ of the natural hybrid orbitals to make a C-O bond. 
Table S3. Cartesian coordinates for the optimized geometries in $\AA$, the ZPEs (a.u.) at the M062X/6-31G level and the total electronic energies at $0 \mathrm{~K}(\mathrm{E} 0 \mathrm{~K}$, a.u.) at the M06-2X/6-311G(d,p) level.

Figure 7a

Graphene

$$
\mathrm{ZPE}=0.690728 \quad \mathrm{Ee}=-2680.622128
$$

\begin{tabular}{|c|c|c|c|}
\hline $\mathrm{C}$ & 9.897123 & 0.000000 & -0.00004 \\
\hline $\mathrm{C}$ & 3.557224 & 3.717486 & 0.00000 \\
\hline $\mathrm{C}$ & 3.557226 & -3.717486 & -0.00001 \\
\hline C & -2.829626 & 0.000000 & -0.0001 \\
\hline C & 7.798609 & 1.240689 & -0.0000 \\
\hline $\mathrm{C}$ & 1.436693 & 4.957995 & 0.0001 \\
\hline $\mathrm{C}$ & 1.422638 & -2.471219 & -0.00004 \\
\hline $\mathrm{C}$ & -4.953918 & 1.233227 & -0.0000 \\
\hline C & 5.679254 & 2.479394 & -0.0000 \\
\hline $\mathrm{C}$ & -0.674997 & 6.195813 & 0.0002 \\
\hline $\mathrm{C}$ & -0.704804 & -1.234847 & -0.0001 \\
\hline C & -7.069567 & 2.456396 & -0.000 \\
\hline $\mathrm{C}$ & -0.674994 & -6.195812 & 0.00 \\
\hline C & -7.069565 & -2.456398 & -0.00 \\
\hline $\mathrm{C}$ & 5.679254 & -2.479393 & -0.00 \\
\hline $\mathrm{C}$ & -0.704803 & 1.234845 & -0.00 \\
\hline $\mathrm{C}$ & -2.804657 & -4.943421 & 0.000 \\
\hline C & -9.209990 & -1.211016 & -0.00 \\
\hline C & 3.543791 & -1.236006 & -0.00 \\
\hline C & -2.825787 & 2.469306 & -0.0 \\
\hline C & -4.936675 & -3.699865 & 0.00 \\
\hline $\mathrm{C}$ & 1.418424 & -0.000002 & -0.00 \\
\hline C & -4.936678 & 3.699864 & -0.00 \\
\hline $\mathrm{C}$ & 1.436695 & -4.957994 & 0.00 \\
\hline $\mathrm{C}$ & -4.953916 & -1.233228 & -0.0000 \\
\hline $\mathrm{C}$ & 7.798609 & -1.240688 & -0.000 \\
\hline $\mathrm{C}$ & 1.422636 & 2.471219 & -0.00 \\
\hline C & -0.696013 & -3.708346 & 0.00 \\
\hline $\mathrm{C}$ & -7.083448 & -0.000001 & -0.0000 \\
\hline $\mathrm{C}$ & 5.666274 & 0.000001 & -0.0000 \\
\hline $\mathrm{C}$ & -0.696015 & 3.708347 & 0.0000 \\
\hline $\mathrm{C}$ & -2.825785 & -2.469306 & -0.0000 \\
\hline C & -9.209991 & 1.211013 & -0.0000 \\
\hline $\mathrm{C}$ & 3.543791 & 1.236008 & -0.0000 \\
\hline $\mathrm{C}$ & -2.804660 & 4.943422 & 0.0001 \\
\hline $\mathrm{C}$ & 2.825790 & -2.469313 & -0.0000 \\
\hline C & -3.543792 & 1.236002 & 0.0000 \\
\hline C & 0.704807 & -1.234841 & -0.0000 \\
\hline $\mathrm{C}$ & -5.679255 & 2.479380 & -0.0000 \\
\hline $\mathrm{C}$ & -1 & 0 & 0.0 \\
\hline
\end{tabular}




\begin{tabular}{|c|c|c|c|}
\hline $\mathrm{C}$ & -1.436671 & -4.957990 & 0.00013 \\
\hline C & -7.798628 & -1.240679 & -0.00007 \\
\hline $\mathrm{C}$ & 4.953913 & -1.233234 & -0.00005 \\
\hline $\mathrm{C}$ & -1.422634 & 2.471218 & 0.00006 \\
\hline C & -3.557211 & -3.717475 & 0.0000 \\
\hline C & -9.897159 & -0.000002 & -0.0000 \\
\hline $\mathrm{C}$ & 2.829625 & 0.000000 & -0.00002 \\
\hline $\mathrm{C}$ & -3.557213 & 3.717475 & 0.0000 \\
\hline $\mathrm{C}$ & -5.679253 & -2.479381 & -0.00003 \\
\hline C & 0.704807 & 1.234846 & 0.0000 \\
\hline C & 0.696023 & -3.708348 & 0.000 \\
\hline C & -5.666284 & -0.000001 & -0.000 \\
\hline $\mathrm{C}$ & 7.083434 & 0.000000 & -0.0000 \\
\hline $\mathrm{C}$ & 0.696020 & 3.708348 & 0.000 \\
\hline $\mathrm{C}$ & -1.422633 & -2.471219 & 0.00 \\
\hline C & -7.798629 & 1.240676 & -0.000 \\
\hline $\mathrm{C}$ & 4.953913 & 1.233235 & -0.000 \\
\hline $\mathrm{C}$ & -1.436675 & 4.957991 & 0.00 \\
\hline $\mathrm{C}$ & -3.543792 & -1.236002 & 0.000 \\
\hline C & 2.825789 & 2.469313 & -0.00 \\
\hline C & 9.209962 & -1.211022 & -0.00 \\
\hline $\mathrm{C}$ & 9.209961 & 1.211023 & -0.00 \\
\hline C & 4.936680 & 3.699876 & -0.00 \\
\hline $\mathrm{C}$ & 2.804670 & 4.943433 & 0.00 \\
\hline C & 4.936681 & -3.699876 & -0.00 \\
\hline C & 2.804673 & -4.943432 & 0.0 \\
\hline $\mathrm{C}$ & 7.069555 & 2.456405 & -0.000 \\
\hline C & 0.675017 & 6.195802 & 0.000 \\
\hline C & 0.675020 & -6.195801 & 0.00 \\
\hline C & 7.069555 & -2.456405 & -0.0 \\
\hline $\mathrm{H}$ & 9.754519 & -2.149253 & -0.000 \\
\hline $\mathrm{H}$ & 9.754519 & 2.149253 & -0.000 \\
\hline $\mathrm{H}$ & 5.484221 & 4.638062 & -0.000 \\
\hline $\mathrm{H}$ & 3.353380 & 5.881095 & 0.0001 \\
\hline $\mathrm{H}$ & 5.484225 & -4.638061 & -0.000 \\
\hline $\mathrm{H}$ & 3.353383 & -5.881094 & 0.0001 \\
\hline $\mathrm{H}$ & 7.617545 & 3.394277 & -0.0000 \\
\hline $\mathrm{H}$ & 1.229552 & 7.128561 & 0.0003 \\
\hline $\mathrm{H}$ & 7.617546 & -3.394276 & -0.00007 \\
\hline $\mathrm{H}$ & 1.229554 & -7.128561 & 0.0003 \\
\hline $\mathrm{H}$ & 10.980963 & 0.000001 & -0.0000 \\
\hline $\mathrm{H}$ & -1.229534 & 7.128563 & 0.0003 \\
\hline $\mathrm{H}$ & -3.353373 & 5.881080 & 0.0001 \\
\hline $\mathrm{H}$ & -5.484210 & 4.638050 & -0.0000 \\
\hline $\mathrm{H}$ & -7.6175 & 3.394268 & -0.0000 \\
\hline $\mathrm{H}$ & -9.754547 & 2.149241 & -0.0000 \\
\hline $\mathrm{H}$ & -10. & -0.000002 & -0 \\
\hline
\end{tabular}




$\begin{array}{lllr}\mathrm{H} & -9.754545 & -2.149245 & -0.000071 \\ \mathrm{H} & -7.617545 & -3.394270 & -0.000045 \\ \mathrm{H} & -5.484207 & -4.638051 & 0.000019 \\ \mathrm{H} & -3.353369 & -5.881079 & 0.000165 \\ \mathrm{H} & -1.229532 & -7.128561 & 0.000341\end{array}$

SW Defected Graphene ZPE=0.690337 Ee=-2680.475359

$\begin{array}{lrrr}\text { C } & 5.242307 & 3.644823 & 0.004262 \\ \text { C } & -5.361363 & -3.727153 & 0.006685 \\ \text { C } & -7.433013 & -2.277077 & 0.015117 \\ \text { C } & -0.928137 & -6.031944 & -0.002434 \\ \text { C } & -3.090753 & -4.938130 & -0.000003 \\ \text { C } & -9.418674 & -0.794379 & 0.024674 \\ \text { C } & -3.972901 & -3.802624 & -0.000037 \\ \text { C } & -9.991472 & 0.483829 & 0.027511 \\ \text { C } & -6.057756 & -2.449584 & 0.007743 \\ \text { C } & 0.415400 & -6.009830 & -0.003039 \\ \text { C } & -8.023935 & -0.969601 & 0.017266 \\ \text { C } & -1.710968 & -4.811447 & -0.004350 \\ \text { C } & -3.246074 & -2.583014 & -0.006987 \\ \text { C } & -9.200639 & 1.622931 & 0.023315 \\ \text { C } & -5.270961 & -1.235672 & 0.001806 \\ \text { C } & 1.160884 & -4.766514 & -0.006336 \\ \text { C } & -0.982301 & -3.514761 & -0.010155 \\ \text { C } & -7.202158 & 0.210421 & 0.012472 \\ \text { C } & -1.882042 & -2.393755 & -0.011658 \\ \text { C } & -7.788334 & 1.510934 & 0.015854 \\ \text { C } & -3.909754 & -1.332987 & -0.005849 \\ \text { C } & 2.531322 & -4.835150 & -0.003269 \\ \text { C } & 0.439299 & -3.492729 & -0.011809 \\ \text { C } & -5.795239 & 0.079293 & 0.004638 \\ \text { C } & -1.713534 & -0.925236 & -0.015046 \\ \text { C } & -6.927026 & 2.628968 & 0.012210 \\ \text { C } & -2.993568 & -0.266103 & -0.010060 \\ \text { C } & 3.370221 & -3.677338 & -0.004980 \\ \text { C } & 1.271092 & -2.287563 & -0.018336 \\ \text { C } & -4.909262 & 1.209474 & 0.000627 \\ \text { C } & 0.763762 & -0.952809 & -0.026920 \\ \text { C } & -5.533095 & 2.505488 & 0.005348 \\ \text { C } & -0.523563 & -0.303059 & -0.023381 \\ \text { C } & 2.710087 & -2.395066 & -0.014307 \\ \text { C } & -3.462955 & 1.085019 & -0.006788 \\ \text { C } & 1.669344 & 0.110502 & -0.032634 \\ \text { C } & -4.703474 & 3.652599 & 0.004366 \\ \text { C } & -0.356560 & 1.170485 & -0.026105\end{array}$




\begin{tabular}{|c|c|c|c|}
\hline $\mathrm{C}$ & 5.620261 & -2.625628 & 0.00462 \\
\hline $\mathrm{C}$ & 3.587995 & -1.255580 & -0.01593 \\
\hline $\mathrm{C}$ & -2.639247 & 2.290744 & -0.00844 \\
\hline $\mathrm{C}$ & 3.032015 & 0.036276 & -0.02550 \\
\hline C & -3.323848 & 3.573467 & -0.00122 \\
\hline C & 1.007552 & 1.346482 & -0.0324 \\
\hline $\mathrm{C}$ & 4.992837 & -1.335605 & -0.0046 \\
\hline $\mathrm{C}$ & -1.202820 & 2.316907 & -0.0162 \\
\hline $\mathrm{C}$ & 3.779645 & 1.244154 & $-0.0191 \varepsilon$ \\
\hline C & -2.579653 & 4.804265 & 0.0012 \\
\hline C & 1.714292 & 2.541429 & -0.0246 \\
\hline C & 7.816535 & -1.516633 & 0.02031 \\
\hline $\mathrm{C}$ & 5.778828 & -0.136154 & -0.0001 \\
\hline $\mathrm{C}$ & -0.475729 & 3.603579 & -0.012 \\
\hline $\mathrm{C}$ & 5.168776 & 1.159805 & -0.00 \\
\hline C & -1.217289 & 4.844831 & -0.002 \\
\hline $\mathrm{C}$ & 3.109010 & 2.503771 & -0.01 \\
\hline C & 7.186834 & -0.225064 & 0.0 \\
\hline $\mathrm{C}$ & 0.931664 & 3.710968 & -0.0147 \\
\hline C & 5.942405 & 2.378614 & 0.00 \\
\hline C & -0.480364 & 6.104645 & 0.003 \\
\hline $\mathrm{C}$ & 9.984050 & -0.401743 & 0.03 \\
\hline C & 3.854275 & 3.729961 & -0.0 \\
\hline $\mathrm{C}$ & 7.971924 & 0.979971 & 0.02 \\
\hline C & 1.676023 & 4.962007 & -0.0 \\
\hline $\mathrm{C}$ & 7.329398 & 2.253013 & 0.0 \\
\hline $\mathrm{C}$ & 9.377020 & 0.853574 & 0.0 \\
\hline C & 4.757123 & -3.769982 & 0.0 \\
\hline C & 7.013139 & -2.689859 & 0.0 \\
\hline C & 9.228121 & -1.570451 & 0.0 \\
\hline $\mathrm{C}$ & 0.871007 & 6.171807 & 0.0 \\
\hline C & 3.059308 & 4.950010 & -0.00 \\
\hline $\mathrm{H}$ & 0.986496 & -6.932264 & -0.0 \\
\hline $\mathrm{H}$ & 3.005220 & -5.812838 & 0.00 \\
\hline $\mathrm{H}$ & 9.98 & 1.751799 & 0.0 \\
\hline $\mathrm{H}$ & 5.212662 & -4.756593 & 0.005 \\
\hline $\mathrm{H}$ & 7.507689 & -3.656970 & 0.02 \\
\hline $\mathrm{H}$ & 9.717912 & -2.538344 & 0.03 \\
\hline $\mathrm{H}$ & 1.375768 & 7.132320 & 0.0092 \\
\hline $\mathrm{H}$ & 3.586819 & 5.900258 & 0.0 \\
\hline $\mathrm{H}$ & 7.952597 & 3.142665 & 0.0244 \\
\hline $\mathrm{H}$ & 5.839872 & 4.552229 & 0.01 \\
\hline $\mathrm{H}$ & 11.066008 & -0.467952 & 0.0481 \\
\hline $\mathrm{H}$ & -3.147879 & 5.730031 & 0.0077 \\
\hline $\mathrm{H}$ & -5.173440 & 4.631627 & 0.008 \\
\hline $\mathrm{H}$ & -7.360857 & 3.624974 & 0.015 \\
\hline 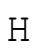 & -9.653 & 2.608736 & 0 \\
\hline
\end{tabular}




$\begin{array}{rrrr}\mathrm{H} & -11.070863 & 0.580427 & 0.033202 \\ \mathrm{H} & -10.057890 & -1.671040 & 0.028336 \\ \mathrm{H} & -8.095520 & -3.137647 & 0.019793 \\ \mathrm{H} & -5.960640 & -4.632239 & 0.012138 \\ \mathrm{H} & -3.509342 & -5.940407 & 0.004194 \\ \mathrm{H} & -1.467611 & -6.973135 & 0.000250 \\ \mathrm{H} & -1.070928 & 7.015406 & 0.009556\end{array}$

\section{Graphene-O (Singlet) $\mathrm{ZPE}=0.696920 \quad \mathrm{Ee}=\quad-2755.747453$}

$\begin{array}{lrrr}\text { C } & -7.061894 & 2.484174 & -0.233158 \\ \text { C } & 5.674957 & 2.506405 & -0.121434 \\ \text { C } & -0.753706 & -1.222900 & 0.564271 \\ \text { C } & -2.801597 & 4.964238 & 0.042360 \\ \text { C } & -9.193999 & 1.239092 & -0.417460 \\ \text { C } & 3.545912 & 1.265498 & 0.059938 \\ \text { C } & -2.828493 & -2.461326 & 0.110926 \\ \text { C } & -4.931384 & 3.725703 & -0.074649 \\ \text { C } & 1.447034 & 0.044483 & 0.308318 \\ \text { C } & -4.932219 & -3.681355 & -0.155575 \\ \text { C } & 1.435223 & 4.975481 & 0.095016 \\ \text { C } & -4.956629 & 1.260745 & -0.056170 \\ \text { C } & 7.783296 & 1.269974 & -0.296701 \\ \text { C } & 1.451893 & -2.480146 & 0.266164 \\ \text { C } & -0.695155 & 3.725703 & 0.152734 \\ \text { C } & -7.069062 & 0.030181 & -0.243272 \\ \text { C } & 5.659821 & 0.030911 & -0.116492 \\ \text { C } & -0.705364 & -3.690767 & 0.205559 \\ \text { C } & -2.829759 & 2.489584 & 0.098113 \\ \text { C } & -9.181031 & -1.182581 & -0.442961 \\ \text { C } & 3.552862 & -1.214240 & 0.083111 \\ \text { C } & -2.802172 & -4.923825 & -0.040647 \\ \text { C } & 3.556647 & 3.740490 & 0.022853 \\ \text { C } & -2.836336 & 0.030879 & 0.156228 \\ \text { C } & 9.873076 & 0.032003 & -0.487436 \\ \text { C } & 3.557631 & -3.705482 & -0.038319 \\ \text { C } & 1.422939 & 2.486818 & 0.182480 \\ \text { C } & -4.940228 & -1.209400 & -0.060792 \\ \text { C } & 7.782547 & -1.211165 & -0.322284 \\ \text { C } & 1.433889 & -4.935676 & 0.057394 \\ \text { C } & -0.720422 & 1.250925 & 0.290814 \\ \text { C } & -7.050071 & -2.430780 & -0.286984 \\ \text { C } & 5.670563 & -2.458656 & -0.166204 \\ \text { C } & -0.674645 & -6.173129 & -0.002771 \\ \text { C } & -1.435223 & 4.975481 & 0.095015 \\ \text { C } & -7.783296 & 1.269974 & -0.296705 \\ \text { C } & 4.956629 & 1.260745 & -0.056173\end{array}$




\begin{tabular}{|c|c|c|c|}
\hline $\mathrm{C}$ & -1.451894 & -2.480145 & 0.26618 \\
\hline $\mathrm{C}$ & -3.556647 & 3.740491 & 0.02285 \\
\hline $\mathrm{C}$ & -9.873075 & 0.032003 & -0.48744 \\
\hline $\mathrm{C}$ & 2.836336 & 0.030879 & 0.15621 \\
\hline C & -3.557631 & -3.705481 & -0.03831 \\
\hline $\mathrm{C}$ & -5.674957 & 2.506405 & -0.1214 \\
\hline $\mathrm{C}$ & 0.753711 & -1.222899 & 0.5 \\
\hline $\mathrm{C}$ & 0.695155 & 3.725703 & 0.1527 \\
\hline $\mathrm{C}$ & -5.659822 & 0.030911 & -0.116 \\
\hline $\mathrm{C}$ & 7.069062 & 0.030180 & -0.2 \\
\hline $\mathrm{C}$ & 0.705360 & -3.690766 & 0.2055 \\
\hline $\mathrm{C}$ & -1.422939 & 2.486818 & 0.1 \\
\hline $\mathrm{C}$ & -7.782547 & -1.211165 & -0.322 \\
\hline $\mathrm{C}$ & 4.940227 & -1.209400 & -0.06 \\
\hline $\mathrm{C}$ & -1.433891 & -4.935676 & 0.05 \\
\hline $\mathrm{C}$ & -3.545911 & 1.265499 & 0.05 \\
\hline $\mathrm{C}$ & 2.828493 & -2.461327 & 0.1 \\
\hline C & 2.829758 & 2.489584 & 0.0 \\
\hline C & -3.552864 & -1.214240 & 0.08 \\
\hline C & 9.181032 & -1.182581 & -0.44 \\
\hline $\mathrm{C}$ & 2.802170 & -4.923825 & -0.04 \\
\hline $\mathrm{C}$ & 0.720419 & 1.250925 & 0.25 \\
\hline $\mathrm{C}$ & -5.670563 & -2.458656 & -0.16 \\
\hline $\mathrm{C}$ & 7.050071 & -2.430780 & -0.286 \\
\hline $\mathrm{C}$ & 0.674644 & -6.173129 & -0.00 \\
\hline $\mathrm{C}$ & -1.447035 & 0.044481 & 0.3 \\
\hline $\mathrm{C}$ & 4.932220 & -3.681355 & -0.1555 \\
\hline $\mathrm{C}$ & 0.674772 & 6.213448 & 0.081 \\
\hline $\mathrm{C}$ & 7.061894 & 2.484174 & -0.233 \\
\hline $\mathrm{C}$ & 4.931383 & 3.725703 & -0.0 \\
\hline $\mathrm{C}$ & 2.801597 & 4.964237 & 0.02 \\
\hline $\mathrm{C}$ & 9.194000 & 1.239092 & -0.417 \\
\hline $\mathrm{H}$ & 7.608523 & 3.421820 & -0.27 \\
\hline $\mathrm{H}$ & 5.474643 & 4.664818 & -0.128 \\
\hline $\mathrm{H}$ & 9.736907 & 2.177473 & -0.4 \\
\hline $\mathrm{C}$ & -0.674772 & 6.213448 & 0.08 \\
\hline $\mathrm{H}$ & -9.736906 & 2.177474 & -0.45 \\
\hline $\mathrm{H}$ & -7.608522 & 3.421820 & -0.27 \\
\hline $\mathrm{H}$ & -5.474644 & 4.664817 & -0.1280 \\
\hline $\mathrm{H}$ & -3.346430 & 5.902987 & -0.0 \\
\hline $\mathrm{H}$ & -1.229986 & 7.145526 & 0.0611 \\
\hline $\mathrm{H}$ & 1.229985 & 7.145527 & 0.0 \\
\hline $\mathrm{H}$ & 3.346430 & 5.902987 & -0.0027 \\
\hline O & 0.000006 & -1.246192 & 1.8550 \\
\hline $\mathrm{H}$ & 10.952840 & 0.027895 & -0.5 \\
\hline $\mathrm{H}$ & 9.723089 & -2.120458 & -0.50 \\
\hline $\mathrm{H}$ & 7.59 & -3.366 & -0 \\
\hline
\end{tabular}




$\begin{array}{lrrr}\mathrm{H} & 5.478528 & -4.615749 & -0.247162 \\ \mathrm{H} & 3.340316 & -5.862046 & -0.142469 \\ \mathrm{H} & 1.230530 & -7.102696 & -0.067758 \\ \mathrm{H} & -1.230532 & -7.102695 & -0.067754 \\ \mathrm{H} & -3.340320 & -5.862045 & -0.142463 \\ \mathrm{H} & -5.478526 & -4.615749 & -0.247162 \\ \mathrm{H} & -7.596462 & -3.366649 & -0.362683 \\ \mathrm{H} & -9.723088 & -2.120458 & -0.503006 \\ \mathrm{H} & -10.952839 & 0.027894 & -0.580061\end{array}$

\section{Graphene-O (Triplet) $\mathrm{ZPE}=0.695011 \quad \mathrm{Ee}=\quad-2755.764607$}

$\begin{array}{lrrr}\text { C } & -7.072305 & 2.477183 & -0.227983 \\ \text { C } & 5.674025 & 2.498949 & -0.118444 \\ \text { C } & -0.753939 & -1.226162 & 0.564301 \\ \text { C } & -2.803498 & 4.954626 & 0.031589 \\ \text { C } & -9.210235 & 1.244802 & -0.405343 \\ \text { C } & 3.546535 & 1.261182 & 0.060964 \\ \text { C } & -2.847441 & -2.463717 & 0.111030 \\ \text { C } & -4.936007 & 3.715114 & -0.077071 \\ \text { C } & 1.451121 & 0.040127 & 0.309555 \\ \text { C } & -4.963264 & -3.667145 & -0.148957 \\ \text { C } & 1.436087 & 4.966628 & 0.082043 \\ \text { C } & -4.963393 & 1.257210 & -0.052942 \\ \text { C } & 7.788396 & 1.271742 & -0.287099 \\ \text { C } & 1.447674 & -2.483997 & 0.263221 \\ \text { C } & -0.697269 & 3.717615 & 0.144243 \\ \text { C } & -7.077202 & 0.029989 & -0.232879 \\ \text { C } & 5.671395 & 0.027508 & -0.108851 \\ \text { C } & -0.725259 & -3.678940 & 0.199012 \\ \text { C } & -2.834784 & 2.482502 & 0.095691 \\ \text { C } & -9.209133 & -1.176389 & -0.427551 \\ \text { C } & 3.559870 & -1.222411 & 0.087523 \\ \text { C } & -2.818128 & -4.905002 & -0.049740 \\ \text { C } & 3.557041 & 3.731170 & 0.018182 \\ \text { C } & -2.836310 & 0.027065 & 0.159506 \\ \text { C } & 9.893934 & 0.041343 & -0.471844 \\ \text { C } & 3.558748 & -3.697325 & -0.032752 \\ \text { C } & 1.419909 & 2.481312 & 0.179253 \\ \text { C } & -4.951049 & -1.216482 & -0.053370 \\ \text { C } & 7.798957 & -1.215276 & -0.309508 \\ \text { C } & 1.435809 & -4.915809 & 0.038599 \\ \text { C } & -0.720123 & 1.249461 & 0.289012 \\ \text { C } & -7.086496 & -2.424298 & -0.275356 \\ \text { C } & 5.674418 & -2.461919 & -0.155774 \\ \text { C } & -0.675795 & -6.149148 & -0.041023 \\ \text { C } & -1.436093 & 4.966625 & 0.082064 \\ & & & \end{array}$




\begin{tabular}{|c|c|c|c|}
\hline C & -7.788396 & 1.271737 & -0.287099 \\
\hline C & 4.963385 & 1.257215 & -0.052971 \\
\hline C & -1.447678 & -2.483981 & 0.2632 \\
\hline $\mathrm{C}$ & -3.557042 & 3.731159 & 0.01821 \\
\hline $\mathrm{C}$ & -9.893935 & 0.041334 & -0.47187 \\
\hline C & 2.836312 & 0.027079 & 0.1594 \\
\hline C & -3.558747 & -3.697304 & -0.03280 \\
\hline C & -5.674030 & 2.498949 & -0.1184 \\
\hline $\mathrm{C}$ & 0.754020 & -1.226154 & 0.56390 \\
\hline C & 0.697263 & 3.717619 & 0.1442 \\
\hline C & -5.671398 & 0.027503 & -0.1088 \\
\hline $\mathrm{C}$ & 7.077200 & 0.029995 & -0.2328 \\
\hline $\mathrm{C}$ & 0.725261 & -3.678965 & 0.1990 \\
\hline $\mathrm{C}$ & -1.419921 & 2.481305 & 0.17925 \\
\hline $\mathrm{C}$ & -7.798962 & -1.215273 & -0.3095 \\
\hline $\mathrm{C}$ & 4.951061 & -1.216484 & -0.053 \\
\hline $\mathrm{C}$ & -1.435812 & -4.915794 & 0.0385 \\
\hline $\mathrm{C}$ & -3.546543 & 1.261158 & 0.061 \\
\hline $\mathrm{C}$ & 2.847446 & -2.463731 & 0.11 \\
\hline C & 2.834787 & 2.482516 & 0.05 \\
\hline $\mathrm{C}$ & -3.559868 & -1.222415 & 0.087 \\
\hline $\mathrm{C}$ & 9.209130 & -1.176381 & -0.42 \\
\hline $\mathrm{C}$ & 2.818138 & -4.905019 & -0.049 \\
\hline $\mathrm{C}$ & 0.720118 & 1.249489 & 0.288 \\
\hline $\mathrm{C}$ & -5.674418 & -2.461910 & -0.155 \\
\hline $\mathrm{C}$ & 7.086500 & -2.424300 & -0.2753 \\
\hline $\mathrm{C}$ & 0.675791 & -6.149154 & -0.04 \\
\hline $\mathrm{C}$ & -1.451126 & 0.040122 & $0.30 s$ \\
\hline $\mathrm{C}$ & 4.963268 & -3.667155 & -0.1489 \\
\hline C & 0.674911 & 6.204061 & 0.06 \\
\hline $\mathrm{C}$ & 7.072306 & 2.477186 & -0.2279 \\
\hline $\mathrm{C}$ & 4.936009 & 3.715121 & -0.077 \\
\hline $\mathrm{C}$ & 2.803490 & 4.954634 & 0.03 \\
\hline $\mathrm{C}$ & 9.210241 & 1.244811 & -0.4053 \\
\hline $\mathrm{H}$ & 7.614891 & 3.417067 & -0.272 \\
\hline $\mathrm{H}$ & 5.479098 & 4.654200 & -0.1322 \\
\hline $\mathrm{H}$ & 9.746650 & 2.186767 & -0.443 \\
\hline $\mathrm{C}$ & -0.674921 & 6.204060 & 0.062 \\
\hline $\mathrm{H}$ & -9.746652 & 2.186754 & -0.4436 \\
\hline $\mathrm{H}$ & -7.614893 & 3.417063 & -0.2724 \\
\hline $\mathrm{H}$ & -5.479086 & 4.654200 & -0.1322 \\
\hline $\mathrm{H}$ & -3.348604 & 5.893070 & -0.0163 \\
\hline $\mathrm{H}$ & -1.229677 & 7.136331 & 0.0388 \\
\hline $\mathrm{H}$ & 1.229666 & 7.136331 & 0.0388 \\
\hline $\mathrm{H}$ & 3.348595 & 5.893077 & -0.016 \\
\hline O & -0.000032 & -1.245534 & 1.8539 \\
\hline $\mathrm{H}$ & 10.974042 & 0.041866 & -0.561 \\
\hline
\end{tabular}




$\begin{array}{lrrr}\mathrm{H} & 9.754588 & -2.112237 & -0.484662 \\ \mathrm{H} & 7.630837 & -3.361405 & -0.345646 \\ \mathrm{H} & 5.505918 & -4.603284 & -0.242430 \\ \mathrm{H} & 3.353262 & -5.844643 & -0.153740 \\ \mathrm{H} & 1.229251 & -7.079096 & -0.119772 \\ \mathrm{H} & -1.229254 & -7.079091 & -0.119822 \\ \mathrm{H} & -3.353238 & -5.844629 & -0.153841 \\ \mathrm{H} & -5.505921 & -4.603268 & -0.242493 \\ \mathrm{H} & -7.630833 & -3.361402 & -0.345691 \\ \mathrm{H} & -9.754578 & -2.112252 & -0.484709 \\ \mathrm{H} & -10.974041 & 0.041875 & -0.561757\end{array}$

SW Defected Graphene-O (Singlet) ZPE $=0.694797 \quad \mathrm{Ee}=\quad-2755.633532$

$\begin{array}{lrrr}\text { C } & -5.013742 & 3.665528 & 0.285681 \\ \mathrm{C} & 5.093883 & -3.721368 & 0.491972 \\ \mathrm{C} & 7.148673 & -2.329747 & 1.009665 \\ \mathrm{C} & 0.841855 & -5.989836 & -0.695190 \\ \mathrm{C} & 2.915005 & -4.893503 & -0.168265 \\ \mathrm{C} & 9.125275 & -0.893059 & 1.450729 \\ \mathrm{C} & 3.760591 & -3.766095 & 0.106314 \\ \mathrm{C} & 9.721391 & 0.368105 & 1.525634 \\ \mathrm{C} & 5.813569 & -2.465909 & 0.666993 \\ \mathrm{C} & -0.498809 & -6.003975 & -0.751396 \\ \mathrm{C} & 7.766479 & -1.036918 & 1.108309 \\ \mathrm{C} & 1.588713 & -4.754293 & -0.525321 \\ \mathrm{C} & 3.080848 & -2.535320 & -0.115722 \\ \mathrm{C} & 8.994778 & 1.523668 & 1.260613 \\ \mathrm{C} & 5.090938 & -1.240270 & 0.439706 \\ \mathrm{C} & -1.253404 & -4.769661 & -0.675785 \\ \mathrm{C} & 0.871581 & -3.451568 & -0.708483 \\ \mathrm{C} & 7.015795 & 0.156828 & 0.842922 \\ \mathrm{C} & 1.792690 & -2.315557 & -0.576963 \\ \mathrm{C} & 7.628153 & 1.442219 & 0.911796 \\ \mathrm{C} & 3.768646 & -1.310817 & 0.087320 \\ \mathrm{C} & -2.595246 & -4.839481 & -0.395187 \\ \mathrm{C} & -0.543218 & -3.491093 & -0.833728 \\ \mathrm{C} & 5.644519 & 0.054964 & 0.498386 \\ \mathrm{C} & 1.734521 & -0.832668 & -0.697831 \\ \mathrm{C} & 6.830729 & 2.580484 & 0.624946 \\ \mathrm{C} & 2.953044 & -0.221113 & -0.201761 \\ \mathrm{C} & -3.420773 & -3.680820 & -0.194464 \\ \mathrm{C} & -1.466035 & -2.349650 & -0.955753 \\ \mathrm{C} & 4.836908 & 1.202276 & 0.196140 \\ \mathrm{C} & -1.106716 & -1.105190 & -1.454841 \\ \mathrm{C} & 5.483916 & 2.483414 & 0.277862 \\ \mathrm{C} & 0.866072 & -0.125556 & -1.422074\end{array}$




\begin{tabular}{|c|c|c|c|}
\hline $\mathrm{C}$ & -2.813788 & -2.412013 & -0.491808 \\
\hline $\mathrm{C}$ & 3.447183 & 1.100814 & -0.18340 \\
\hline $\mathrm{C}$ & -1.735967 & 0.088885 & -1.13848 \\
\hline $\mathrm{C}$ & 4.694039 & 3.634680 & 0.02016 \\
\hline $\mathrm{C}$ & 0.427908 & 1.238091 & -1.19001 \\
\hline $\mathrm{C}$ & -5.527065 & -2.553355 & 0.41982 \\
\hline $\mathrm{C}$ & -3.594824 & -1.229704 & -0.342 \\
\hline $\mathrm{C}$ & 2.651977 & 2.304977 & -0.4617 \\
\hline $\mathrm{C}$ & -3.018025 & 0.041025 & -0.593 \\
\hline $\mathrm{C}$ & 3.350698 & 3.573421 & -0.296 \\
\hline $\mathrm{C}$ & -0.970812 & 1.316105 & -1.152 \\
\hline $\mathrm{C}$ & -4.923063 & -1.280539 & 0.144 \\
\hline $\mathrm{C}$ & 1.258648 & 2.353107 & -0.7909 \\
\hline $\mathrm{C}$ & -3.698365 & 1.251379 & -0.302 \\
\hline $\mathrm{C}$ & 2.636516 & 4.812178 & -0.41 \\
\hline $\mathrm{C}$ & -1.643811 & 2.510729 & -0.7832 \\
\hline $\mathrm{C}$ & -7.586239 & -1.395728 & 1.09 \\
\hline C & -5.638392 & -0.073658 & 0.37 \\
\hline C & 0.540409 & 3.625655 & -0.6996 \\
\hline C & -5.014337 & 1.197968 & 0.1631 \\
\hline $\mathrm{C}$ & 1.283501 & 4.860668 & -0.55 \\
\hline $\mathrm{C}$ & -3.001411 & 2.493654 & -0.43 \\
\hline $\mathrm{C}$ & -6.968527 & -0.126159 & 0.85 \\
\hline $\mathrm{C}$ & -0.858998 & 3.695030 & -0.677 \\
\hline $\mathrm{C}$ & -5.719758 & 2.424518 & 0.44 \\
\hline $\mathrm{C}$ & 0.559296 & 6.121092 & -0.500 \\
\hline $\mathrm{C}$ & -9.612760 & -0.235456 & 1.79 \\
\hline $\mathrm{C}$ & -3.689373 & 3.717804 & -0.114 \\
\hline $\mathrm{C}$ & -7.682989 & 1.092316 & 1.106 \\
\hline $\mathrm{C}$ & -1.571496 & 4.944050 & -0.44 \\
\hline $\mathrm{C}$ & -7.032208 & 2.343841 & $0.89^{\circ}$ \\
\hline $\mathrm{C}$ & -9.008214 & 1.005510 & 1.57 \\
\hline $\mathrm{C}$ & -4.737677 & -3.734900 & 0.22 \\
\hline $\mathrm{C}$ & -6.844296 & -2.583634 & 0.875 \\
\hline $\mathrm{C}$ & -8.922614 & -1.418189 & 1.5 \\
\hline $\mathrm{C}$ & -0.787192 & 6.165698 & -0.4372 \\
\hline $\mathrm{C}$ & -2.922266 & 4.936972 & -0.197 \\
\hline $\mathrm{H}$ & -1.051814 & -6.935565 & -0.805 \\
\hline $\mathrm{H}$ & -3.048743 & -5.818583 & -0.2697 \\
\hline $\mathrm{H}$ & -9.559702 & 1.919974 & 1.7 \\
\hline $\mathrm{H}$ & -5.196318 & -4.700184 & 0.4180 \\
\hline $\mathrm{H}$ & -7.318064 & -3.539505 & 1.08 \\
\hline $\mathrm{H}$ & -9.399017 & -2.375534 & 1.746 \\
\hline $\mathrm{H}$ & -1.312109 & 7.111508 & -0.35 \\
\hline $\mathrm{H}$ & -3.431190 & 5.878063 & -0.00 \\
\hline $\mathrm{H}$ & -7.583204 & 3.254790 & 1.11 \\
\hline $\mathrm{H}$ & - & 4 & \\
\hline
\end{tabular}




$\begin{array}{rrrr}\mathrm{H} & -10.634939 & -0.272620 & 2.150012 \\ \mathrm{O} & 0.051410 & -0.938903 & -2.217643 \\ \mathrm{H} & 3.203518 & 5.732951 & -0.315659 \\ \mathrm{H} & 5.162611 & 4.610611 & 0.107983 \\ \mathrm{H} & 7.287846 & 3.564466 & 0.679518 \\ \mathrm{H} & 9.470442 & 2.496910 & 1.317555 \\ \mathrm{H} & 10.769180 & 0.444821 & 1.791963 \\ \mathrm{H} & 9.712453 & -1.782063 & 1.655789 \\ \mathrm{H} & 7.761504 & -3.205626 & 1.201949 \\ \mathrm{H} & 5.642798 & -4.645384 & 0.647985 \\ \mathrm{H} & 3.316708 & -5.898003 & -0.073901 \\ \mathrm{H} & 1.409005 & -6.914477 & -0.708579 \\ \mathrm{H} & 1.148140 & 7.032006 & -0.471437\end{array}$

SW Defected Graphene-O (Triplet) ZPE $=0.693608 \quad \mathrm{Ee}=\quad-2755.654192$

\begin{tabular}{|c|c|c|c|}
\hline $\mathrm{C}$ & -5.047124 & 3.650756 & 0.276030 \\
\hline C & 5.107102 & -3.709830 & 0.501915 \\
\hline C & 7.174108 & -2.320556 & 0.989673 \\
\hline $\mathrm{C}$ & 0.842711 & -5.980699 & -0.640995 \\
\hline $\mathrm{C}$ & 2.920790 & -4.883734 & -0.135625 \\
\hline C & 9.167669 & -0.897225 & 1.400174 \\
\hline C & 3.769347 & -3.756213 & 0.125852 \\
\hline $\mathrm{C}$ & 9.767246 & 0.356551 & 1.458893 \\
\hline $\mathrm{C}$ & 5.828027 & -2.454812 & 0.662196 \\
\hline $\mathrm{C}$ & -0.498262 & -5.993790 & -0.697353 \\
\hline $\mathrm{C}$ & 7.792660 & -1.036998 & 1.071316 \\
\hline $\mathrm{C}$ & 1.591981 & -4.745056 & -0.487583 \\
\hline $\mathrm{C}$ & 3.091093 & -2.526748 & -0.098114 \\
\hline C & 9.040863 & 1.517697 & 1.193577 \\
\hline $\mathrm{C}$ & 5.111756 & -1.232863 & 0.437738 \\
\hline C & -1.252162 & -4.758626 & -0.638998 \\
\hline $\mathrm{C}$ & 0.876771 & -3.442985 & -0.679046 \\
\hline C & 7.046031 & 0.155193 & 0.807582 \\
\hline $\mathrm{C}$ & 1.797864 & -2.309252 & -0.557197 \\
\hline C & 7.667354 & 1.444023 & 0.860264 \\
\hline $\mathrm{C}$ & 3.777433 & -1.302637 & 0.096561 \\
\hline $\mathrm{C}$ & -2.598284 & -4.827859 & -0.370344 \\
\hline C & -0.541612 & -3.483315 & -0.804773 \\
\hline $\mathrm{C}$ & 5.669786 & 0.056599 & 0.480352 \\
\hline C & 1.742579 & -0.829272 & -0.680499 \\
\hline C & 6.879916 & 2.578553 & 0.576843 \\
\hline $\mathrm{C}$ & 2.965380 & -0.219005 & -0.190652 \\
\hline $\mathrm{C}$ & -3.427873 & -3.671213 & -0.185673 \\
\hline C & -1.462813 & -2.343544 & -0.933070 \\
\hline C & 4.858631 & 1.208584 & 0.177022 \\
\hline $\mathrm{C}$ & -1.109461 & -1.106679 & -1.437340 \\
\hline
\end{tabular}




\begin{tabular}{|c|c|c|c|}
\hline $\mathrm{C}$ & 5.503835 & 2.487261 & 0.23968 \\
\hline $\mathrm{C}$ & 0.869742 & -0.122860 & -1.39804 \\
\hline $\mathrm{C}$ & -2.823479 & -2.405376 & -0.4774 \\
\hline $\mathrm{C}$ & 3.464611 & 1.108137 & -0.1864 \\
\hline C & -1.744620 & 0.092280 & -1.1295 \\
\hline $\mathrm{C}$ & 4.730627 & 3.624117 & -0.0112 \\
\hline $\mathrm{C}$ & 0.432884 & 1.241333 & -1.174 \\
\hline $\mathrm{C}$ & -5.541267 & -2.547315 & 0.4017 \\
\hline $\mathrm{C}$ & -3.603497 & -1.226516 & -0.340 \\
\hline $\mathrm{C}$ & 2.677443 & 2.305561 & -0.462 \\
\hline $\mathrm{C}$ & -3.027150 & 0.043988 & -0.595 \\
\hline $\mathrm{C}$ & 3.359070 & 3.559912 & -0.31 \\
\hline C & -0.977753 & 1.317841 & -1.1402 \\
\hline C & -4.941937 & -1.278854 & 0.131 \\
\hline $\mathrm{C}$ & 1.248960 & 2.350891 & -0.7 \\
\hline $\mathrm{C}$ & -3.712031 & 1.256907 & -0.30 \\
\hline $\mathrm{C}$ & 2.646015 & 4.792737 & -0.39 \\
\hline C & -1.640027 & 2.507449 & -0.77 \\
\hline C & -7.614995 & -1.395605 & 1.05 \\
\hline $\mathrm{C}$ & -5.665229 & -0.070524 & 0.3 \\
\hline $\mathrm{C}$ & 0.547672 & 3.609694 & -0.65 \\
\hline $\mathrm{C}$ & -5.039686 & 1.202590 & 0.1 \\
\hline $\mathrm{C}$ & 1.283777 & 4.839567 & -0.4 \\
\hline $\mathrm{C}$ & -3.025671 & 2.491385 & -0.43 \\
\hline $\mathrm{C}$ & -6.996866 & -0.124211 & 0.81 \\
\hline $\mathrm{C}$ & -0.871063 & 3.681752 & -0.63 \\
\hline $\mathrm{C}$ & -5.737287 & 2.423490 & 0.42 \\
\hline $\mathrm{C}$ & 0.560410 & 6.096512 & -0.40 \\
\hline $\mathrm{C}$ & -9.662136 & -0.235797 & 1.72 \\
\hline $\mathrm{C}$ & -3.699671 & 3.707192 & -0.1 \\
\hline $\mathrm{C}$ & -7.718527 & 1.097202 & 1.05 \\
\hline $\mathrm{C}$ & -1.574411 & 4.924771 & $-0.3 \varepsilon$ \\
\hline $\mathrm{C}$ & -7.081331 & 2.336022 & 0.8 \\
\hline $\mathrm{C}$ & -9.061843 & 1.004435 & 1.51 \\
\hline $\mathrm{C}$ & -4.754373 & -3.724523 & 0.2 \\
\hline $\mathrm{C}$ & -6.875687 & -2.576061 & 0.84 \\
\hline $\mathrm{C}$ & -8.964511 & -1.418164 & 1.50 \\
\hline $\mathrm{C}$ & -0.786920 & 6.141861 & -0.34 \\
\hline $\mathrm{C}$ & -2.934649 & 4.919813 & -0.161 \\
\hline $\mathrm{H}$ & -1.051877 & -6.925631 & -0.7 \\
\hline $\mathrm{H}$ & -3.050435 & -5.807088 & -0.2415 \\
\hline $\mathrm{H}$ & -9.616031 & 1.918914 & 1.6 \\
\hline $\mathrm{H}$ & -5.212510 & -4.689891 & $0.414^{\circ}$ \\
\hline $\mathrm{H}$ & -7.348356 & -3.533447 & 1.0456 \\
\hline $\mathrm{H}$ & -9.439775 & -2.376863 & 1 \\
\hline $\mathrm{H}$ & -1.309028 & 7.086443 & -0.2 \\
\hline $\mathrm{H}$ & -3.44 & 14 & 0 \\
\hline
\end{tabular}




$\begin{array}{rrrr}\mathrm{H} & -7.632167 & 3.249582 & 1.065182 \\ \mathrm{H} & -5.569510 & 4.574043 & 0.509802 \\ \mathrm{H} & -10.688426 & -0.277606 & 2.071423 \\ \mathrm{O} & 0.052556 & -0.939025 & -2.188243 \\ \mathrm{H} & 3.210227 & 5.714813 & -0.293340 \\ \mathrm{H} & 5.193611 & 4.603361 & 0.066509 \\ \mathrm{H} & 7.337939 & 3.562488 & 0.617514 \\ \mathrm{H} & 9.522660 & 2.488478 & 1.238794 \\ \mathrm{H} & 10.818321 & 0.432599 & 1.712564 \\ \mathrm{H} & 9.749332 & -1.789844 & 1.604662 \\ \mathrm{H} & 7.783876 & -3.199039 & 1.180237 \\ \mathrm{H} & 5.656485 & -4.633515 & 0.659014 \\ \mathrm{H} & 3.320480 & -5.888459 & -0.034724 \\ \mathrm{H} & 1.408054 & -6.906580 & -0.642460 \\ \mathrm{H} & 1.149459 & 7.006199 & -0.355038\end{array}$

Figure $7 b$

Nanotube ZPE $=\quad 1.184520 \quad \mathrm{Ee}=-5501.434128$

\begin{tabular}{|c|c|c|c|}
\hline C & 5.684729 & -3.713167 & 2.962508 \\
\hline C & 5.002610 & -2.813344 & 3.806246 \\
\hline $\mathrm{C}$ & 5.676336 & -1.735005 & 4.417962 \\
\hline $\mathrm{C}$ & 4.994149 & -0.535797 & 4.709919 \\
\hline $\mathrm{C}$ & 5.674432 & 0.699677 & 4.693921 \\
\hline $\mathrm{C}$ & 4.998121 & 1.887392 & 4.345029 \\
\hline C & 5.681276 & 2.950751 & 3.719874 \\
\hline $\mathrm{C}$ & 5.010062 & 3.799021 & 2.813318 \\
\hline $\mathrm{C}$ & 5.689316 & 4.419387 & 1.745408 \\
\hline $\mathrm{C}$ & 5.017808 & 4.691662 & 0.534489 \\
\hline C & 5.691202 & 4.700180 & -0.704946 \\
\hline C & 5.014723 & 4.331904 & -1.885660 \\
\hline $\mathrm{C}$ & 5.685331 & 3.712767 & -2.962532 \\
\hline $\mathrm{C}$ & 5.003211 & 2.812716 & -3.806105 \\
\hline $\mathrm{C}$ & 5.676725 & 1.734424 & -4.417917 \\
\hline C & 4.994361 & 0.535158 & -4.709795 \\
\hline $\mathrm{C}$ & 5.674470 & -0.700273 & -4.693634 \\
\hline $\mathrm{C}$ & 4.997887 & -1.888021 & -4.344915 \\
\hline $\mathrm{C}$ & 5.680985 & -2.951150 & -3.719451 \\
\hline $\mathrm{C}$ & 5.009514 & -3.799587 & -2.813159 \\
\hline $\mathrm{C}$ & 5.688811 & -4.419630 & -1.745099 \\
\hline C & 5.017094 & -4.692173 & -0.534340 \\
\hline $\mathrm{C}$ & 5.690579 & -4.700448 & 0.705058 \\
\hline C & 5.013961 & -4.332468 & 1.885785 \\
\hline $\mathrm{C}$ & 3.558043 & -2.804117 & 3.799516 \\
\hline $\mathrm{C}$ & 2.847499 & -1.727754 & 4.397887 \\
\hline $\mathrm{C}$ & 3.550926 & -0.534314 & 4.704117 \\
\hline $\mathrm{C}$ & 2.845867 & 0.697434 & 4.676039 \\
\hline
\end{tabular}




\begin{tabular}{|c|c|c|c|}
\hline C & 3.554188 & 1.881963 & 4.33870 \\
\hline C & 2.851818 & 2.933840 & 3.69708 \\
\hline C & 3.564774 & 3.784696 & 2.80717 \\
\hline $\mathrm{C}$ & 2.859536 & 4.380591 & 1.7294 \\
\hline $\mathrm{C}$ & 3.572253 & 4.671463 & 0.5330 \\
\hline C & 2.861426 & 4.655645 & -0.6981 \\
\hline C & 3.569138 & 4.314207 & -1.8811 \\
\hline C & 2.855582 & 3.685968 & $-2.9400 \varepsilon$ \\
\hline $\mathrm{C}$ & 3.558569 & 2.803430 & -3.79936 \\
\hline C & 2.847845 & 1.727262 & -4.3978 \\
\hline C & 3.551133 & 0.533682 & -4.704 \\
\hline $\mathrm{C}$ & 2.845925 & -0.697919 & -4.6760 \\
\hline $\mathrm{C}$ & 3.554038 & -1.882630 & -4.3387 \\
\hline $\mathrm{C}$ & 2.851535 & -2.934382 & -3.6971 \\
\hline $\mathrm{C}$ & 3.564292 & -3.785385 & -2.807 \\
\hline $\mathrm{C}$ & 2.858969 & -4.381159 & -1.729 \\
\hline $\mathrm{C}$ & 3.571564 & -4.672119 & -0.53300 \\
\hline $\mathrm{C}$ & 2.860731 & -4.656214 & 0.6981 \\
\hline $\mathrm{C}$ & 3.568419 & -4.314888 & 1.88 \\
\hline $\mathrm{C}$ & 2.854972 & -3.686516 & 2.94 \\
\hline $\mathrm{C}$ & 1.417646 & -1.729039 & 4.390 \\
\hline $\mathrm{C}$ & 0.706390 & -0.534362 & 4.68 \\
\hline $\mathrm{C}$ & 1.416164 & 0.697963 & 4.667 \\
\hline $\mathrm{C}$ & 0.708753 & 1.884576 & 4.326 \\
\hline $\mathrm{C}$ & 1.421575 & 2.937761 & 3.69 \\
\hline $\mathrm{C}$ & 0.716115 & 3.799214 & 2.8061 \\
\hline $\mathrm{C}$ & 1.428453 & 4.390296 & 1.72 \\
\hline $\mathrm{C}$ & 0.721241 & 4.697007 & 0.533 \\
\hline $\mathrm{C}$ & 1.430076 & 4.666929 & -0.6981 \\
\hline C & 0.719142 & 4.334889 & -1.88 \\
\hline $\mathrm{C}$ & 1.424946 & 3.692486 & -2.9380 \\
\hline $\mathrm{C}$ & 0.711855 & 2.810150 & -3.7923 \\
\hline $\mathrm{C}$ & 1.418015 & 1.728773 & -4.3907 \\
\hline $\mathrm{C}$ & 0.706595 & 0.534183 & -4.6865 \\
\hline $\mathrm{C}$ & 1.416205 & -0.698213 & -4.6674 \\
\hline $\mathrm{C}$ & 0.708618 & -1.884722 & -4.3261 \\
\hline $\mathrm{C}$ & 1.421276 & -2.938115 & -3.6930 \\
\hline $\mathrm{C}$ & 0.715681 & -3.799363 & -2.8061 \\
\hline C & 1.427876 & -4.390715 & -1.7290 \\
\hline $\mathrm{C}$ & 0.720609 & -4.697040 & -0.5336 \\
\hline $\mathrm{C}$ & 1.429372 & -4.667327 & 0.6981 \\
\hline $\mathrm{C}$ & 0.718477 & -4.334986 & 1.8822 \\
\hline $\mathrm{C}$ & 1.424315 & -3.692832 & 2.9381 \\
\hline $\mathrm{C}$ & 0.711339 & -2.810320 & 3.7923 \\
\hline $\mathrm{C}$ & -0.723254 & -0.534133 & 4.6 \\
\hline $\mathrm{C}$ & -1.431150 & 0.698732 & 4.6583 \\
\hline $\mathrm{C}$ & -0.720651 & 1.884784 & 4.32 \\
\hline
\end{tabular}




\begin{tabular}{|c|c|c|c|}
\hline C & -1.425016 & 2.944710 & 3.691067 \\
\hline C & -0.712080 & 3.803439 & 2.80588 \\
\hline C & -1.416746 & 4.409112 & 1.73150 \\
\hline $\mathrm{C}$ & -0.705954 & 4.705625 & 0.53401 \\
\hline $\mathrm{C}$ & -1.414706 & 4.689019 & -0.6994 \\
\hline $\mathrm{C}$ & -0.708536 & 4.341529 & -1.88298 \\
\hline C & -1.421025 & 3.704566 & -2.93932 \\
\hline C & -0.717075 & 2.811605 & -3.78976 \\
\hline $\mathrm{C}$ & -1.429132 & 1.731231 & -4.38 \\
\hline C & -0.723060 & 0.534159 & -4.6800 \\
\hline $\mathrm{C}$ & -1.431125 & -0.698530 & -4.6583 \\
\hline $\mathrm{C}$ & -0.720737 & -1.884714 & -4.32142 \\
\hline $\mathrm{C}$ & -1.425276 & -2.944455 & -3.691 \\
\hline $\mathrm{C}$ & -0.712481 & -3.803401 & -2.80598 \\
\hline $\mathrm{C}$ & -1.417355 & -4.408634 & -1.7314 \\
\hline $\mathrm{C}$ & -0.706638 & -4.705444 & -0.534 \\
\hline $\mathrm{C}$ & -1.415460 & -4.688475 & 0.6993 \\
\hline $\mathrm{C}$ & -0.709212 & -4.341391 & 1.882 \\
\hline $\mathrm{C}$ & -1.421624 & -3.704239 & 2.93 \\
\hline $\mathrm{C}$ & -0.717553 & -2.811529 & 3.78 \\
\hline $\mathrm{C}$ & -1.429485 & -1.731028 & 4.38 \\
\hline $\mathrm{C}$ & -2.860788 & 0.698886 & 4.64 \\
\hline $\mathrm{C}$ & -3.565259 & 1.888175 & 4.309 \\
\hline $\mathrm{C}$ & -2.855569 & 2.947173 & 3.684 \\
\hline $\mathrm{C}$ & -3.555143 & 3.821036 & 2.80 \\
\hline $\mathrm{C}$ & -2.848325 & 4.417510 & 1.730 \\
\hline $\mathrm{C}$ & -3.548079 & 4.737889 & 0.53 \\
\hline $\mathrm{C}$ & -2.846584 & 4.699211 & -0.6991 \\
\hline $\mathrm{C}$ & -3.551054 & 4.366949 & -1.885 \\
\hline C & -2.852059 & 3.709503 & -2.93 \\
\hline $\mathrm{C}$ & -3.561074 & 2.819741 & -3.7831 \\
\hline $\mathrm{C}$ & -2.859024 & 1.731806 & -4.373 \\
\hline $\mathrm{C}$ & -3.568249 & 0.534947 & -4.6630 \\
\hline $\mathrm{C}$ & -2.860829 & -0.698423 & -4.6458 \\
\hline $\mathrm{C}$ & -3.565478 & -1.887580 & -4.30 \\
\hline $\mathrm{C}$ & -2.855739 & -2.946669 & -3.6840 \\
\hline $\mathrm{C}$ & -3.555692 & -3.820238 & -2.8059 \\
\hline $\mathrm{C}$ & -2.848929 & -4.416793 & -1.7301 \\
\hline C & -3.548819 & -4.736565 & -0.5352 \\
\hline $\mathrm{C}$ & -2.847358 & -4.698394 & 0.69 \\
\hline $\mathrm{C}$ & -3.551790 & -4.365926 & 1.8851 \\
\hline $\mathrm{C}$ & -2.852618 & -3.708925 & 2.93 \\
\hline C & -3.561658 & -2.819090 & 3.7831 \\
\hline $\mathrm{C}$ & -2.859433 & -1.731325 & 4.3734 \\
\hline $\mathrm{C}$ & -3.568479 & -0.534314 & 4.6 \\
\hline $\mathrm{C}$ & -5.011294 & 1.884161 & 4.3050 \\
\hline $\mathrm{C}$ & -5.689653 & 2.949768 & 3.6 \\
\hline
\end{tabular}




\begin{tabular}{|c|c|c|c|}
\hline C & -4.998040 & 3.811996 & 2.80208 \\
\hline $\mathrm{C}$ & -4.987870 & 4.725577 & 0.5342 \\
\hline C & -4.992290 & 4.356021 & -1.88250 \\
\hline $\mathrm{C}$ & -5.685041 & 3.708710 & -2.9276 \\
\hline $\mathrm{C}$ & -5.005968 & 2.813539 & -3.7791 \\
\hline $\mathrm{C}$ & -5.694523 & 1.735614 & -4.3728 \\
\hline C & -5.014986 & 0.533767 & -4.6593 \\
\hline $\mathrm{C}$ & -5.696858 & -0.699990 & -4.6481 \\
\hline $\mathrm{C}$ & -5.011491 & -1.883876 & -4.3052 \\
\hline C & -5.690101 & -2.949339 & -3.6795 \\
\hline C & -4.998568 & -3.811619 & -2.802 \\
\hline $\mathrm{C}$ & -5.680421 & -4.410658 & -1.7238 \\
\hline $\mathrm{C}$ & -4.988931 & -4.724802 & -0.534 \\
\hline $\mathrm{C}$ & -5.678086 & -4.689197 & 0.695 \\
\hline $\mathrm{C}$ & -4.993170 & -4.355462 & 1.88 \\
\hline $\mathrm{C}$ & -5.685705 & -3.708200 & 2.928 \\
\hline $\mathrm{C}$ & -5.006472 & -2.813228 & 3.77 \\
\hline $\mathrm{C}$ & -5.694818 & -1.734855 & 4.37 \\
\hline $\mathrm{C}$ & -5.015184 & -0.533467 & 4.659 \\
\hline C & -5.677561 & 4.689342 & -0.69 \\
\hline $\mathrm{H}$ & -6.763876 & 4.673390 & -0.691 \\
\hline $\mathrm{C}$ & -5.680014 & 4.410739 & 1.7 \\
\hline $\mathrm{H}$ & -6.766218 & 4.397345 & 1.71 \\
\hline $\mathrm{C}$ & -5.697092 & 0.700838 & 4.64 \\
\hline $\mathrm{H}$ & -6.782488 & 0.699715 & 4.62 \\
\hline $\mathrm{H}$ & 6.767083 & 2.941789 & 3.71 \\
\hline $\mathrm{H}$ & 6.774665 & 4.409108 & 1.7 \\
\hline $\mathrm{H}$ & 6.776450 & 4.689750 & -0.7046 \\
\hline $\mathrm{H}$ & 6.770917 & 3.703051 & -2.96 \\
\hline $\mathrm{H}$ & 6.760622 & 0.696844 & 4.68 \\
\hline $\mathrm{H}$ & 6.762793 & 1.728131 & -4.41 \\
\hline $\mathrm{H}$ & 6.762409 & -1.728912 & 4.411 \\
\hline $\mathrm{H}$ & 6.760663 & -0.697658 & -4.6857 \\
\hline $\mathrm{H}$ & 6.770321 & -3.703491 & 2.9608 \\
\hline $\mathrm{H}$ & 6.766798 & -2.942215 & -3.71 \\
\hline $\mathrm{H}$ & 6.775830 & -4.690011 & 0.7047 \\
\hline $\mathrm{H}$ & 6.774163 & -4.409320 & -1.7445 \\
\hline $\mathrm{H}$ & -6.766901 & -4.398049 & -1.7147 \\
\hline $\mathrm{H}$ & -6.764680 & -4.674213 & 0.6919 \\
\hline $\mathrm{H}$ & -6.776114 & -2.943898 & -3.6641 \\
\hline $\mathrm{H}$ & -6.771926 & -3.699587 & 2.9142 \\
\hline $\mathrm{H}$ & -6.782528 & -0.698925 & -4.6309 \\
\hline $\mathrm{H}$ & -6.780579 & -1.732152 & 4.3562 \\
\hline $\mathrm{H}$ & -6.780292 & 1.732782 & -4.3556 \\
\hline $\mathrm{H}$ & -6.771287 & 3.699839 & -2.913 \\
\hline $\mathrm{H}$ & -6.775657 & 2.943988 & 3.6629 \\
\hline
\end{tabular}




\begin{tabular}{|c|c|c|c|}
\hline \multicolumn{2}{|c|}{ SW Defected Nanotube ZPE= } & 1.186688 & -5501.322377 \\
\hline $\mathrm{C}$ & -5.714189 & -4.769254 & -0.046535 \\
\hline $\mathrm{C}$ & -6.009659 & -3.814479 & 2.205243 \\
\hline $\mathrm{C}$ & -6.209116 & -1.855383 & 3.684344 \\
\hline $\mathrm{C}$ & -6.290720 & 0.571603 & 4.037292 \\
\hline $\mathrm{C}$ & -6.308942 & 2.836672 & 3.142997 \\
\hline $\mathrm{C}$ & -4.993171 & 1.835042 & -4.929563 \\
\hline $\mathrm{C}$ & -5.396851 & -4.423388 & -2.461900 \\
\hline $\mathrm{C}$ & -4.883071 & -4.740400 & -1.184187 \\
\hline $\mathrm{C}$ & -5.198409 & -4.417513 & 1.217811 \\
\hline $\mathrm{C}$ & -5.451037 & -2.914464 & 3.132207 \\
\hline $\mathrm{C}$ & -5.588234 & -0.652664 & 4.071485 \\
\hline $\mathrm{C}$ & -5.622370 & 1.788927 & 3.797084 \\
\hline $\mathrm{C}$ & -5.605003 & 3.753166 & 2.335714 \\
\hline $\mathrm{C}$ & -5.370340 & 4.689858 & 0.067091 \\
\hline $\mathrm{C}$ & -4.742855 & 4.362273 & -2.287397 \\
\hline $\mathrm{C}$ & -4.397387 & 2.877502 & -4.189678 \\
\hline $\mathrm{C}$ & -4.309629 & 0.622217 & -5.130276 \\
\hline $\mathrm{C}$ & -4.379225 & -1.809783 & -4.855319 \\
\hline $\mathrm{C}$ & -4.585459 & -3.780135 & -3.414606 \\
\hline $\mathrm{C}$ & -3.450141 & -4.726689 & -0.998495 \\
\hline $\mathrm{C}$ & -3.764793 & -4.393800 & 1.396218 \\
\hline $\mathrm{C}$ & -4.016968 & -2.884117 & 3.296331 \\
\hline $\mathrm{C}$ & -4.152437 & -0.614958 & 4.201064 \\
\hline $\mathrm{C}$ & -4.174897 & 1.819305 & 3.905489 \\
\hline $\mathrm{C}$ & -4.166980 & 3.808904 & 2.528988 \\
\hline $\mathrm{C}$ & -3.977865 & 4.755806 & 0.309230 \\
\hline $\mathrm{C}$ & -3.299800 & 4.325796 & -1.991307 \\
\hline $\mathrm{C}$ & -2.976983 & 2.831964 & -3.909062 \\
\hline $\mathrm{C}$ & -2.881562 & 0.603938 & -4.919788 \\
\hline $\mathrm{C}$ & -2.946621 & -1.824564 & -4.673251 \\
\hline $\mathrm{C}$ & -3.152729 & -3.784088 & -3.229718 \\
\hline $\mathrm{C}$ & -2.590637 & -4.411610 & -2.088286 \\
\hline $\mathrm{C}$ & -2.904858 & -4.724604 & 0.313314 \\
\hline $\mathrm{C}$ & -3.198755 & -3.759415 & 2.537148 \\
\hline $\mathrm{C}$ & -3.400075 & -1.791689 & 3.967607 \\
\hline $\mathrm{C}$ & -3.475966 & 0.634295 & 4.249045 \\
\hline $\mathrm{C}$ & -3.448679 & 2.912278 & 3.370124 \\
\hline $\mathrm{C}$ & -3.407691 & 4.470803 & 1.578571 \\
\hline $\mathrm{C}$ & -2.969159 & 4.686326 & -0.667233 \\
\hline $\mathrm{C}$ & -2.391522 & 3.680122 & -2.935975 \\
\hline $\mathrm{C}$ & -2.211023 & 1.766565 & -4.478748 \\
\hline $\mathrm{C}$ & -2.194594 & -0.639176 & -4.872178 \\
\hline $\mathrm{C}$ & -2.333514 & -2.909024 & -3.994221 \\
\hline $\mathrm{C}$ & -1.171355 & -4.422358 & -1.904038 \\
\hline $\mathrm{C}$ & -1.484073 & -4.727212 & 0.499127 \\
\hline $\mathrm{C}$ & -1.779008 & -3.750783 & 2.717169 \\
\hline
\end{tabular}




\begin{tabular}{|c|c|c|c|}
\hline $\mathrm{C}$ & -1.982918 & -1.766109 & 4.12354 \\
\hline $\mathrm{C}$ & -2.054629 & 0.670214 & $4.3462 \varepsilon$ \\
\hline $\mathrm{C}$ & -2.001055 & 2.927674 & 3.3706 \\
\hline $\mathrm{C}$ & -1.994990 & 4.414802 & 1.4825 \\
\hline C & -0.483153 & 4.633957 & -0.474 \\
\hline $\mathrm{C}$ & -0.934964 & 3.714359 & -2.8580 \\
\hline $\mathrm{C}$ & -0.791401 & 1.761469 & -4.349 \\
\hline $\mathrm{C}$ & -0.779518 & -0.654720 & -4.7054 \\
\hline $\mathrm{C}$ & -0.916585 & -2.923960 & -3.815 \\
\hline $\mathrm{C}$ & -0.329296 & -3.811053 & -2.868 \\
\hline $\mathrm{C}$ & -0.620757 & -4.741103 & -0.628 \\
\hline $\mathrm{C}$ & -0.933645 & -4.387470 & 1.7 \\
\hline C & -1.192004 & -2.845275 & 3.63 \\
\hline $\mathrm{C}$ & -1.334213 & -0.549129 & 4.45 \\
\hline $\mathrm{C}$ & -1.334882 & 1.861921 & 4.02 \\
\hline $\mathrm{C}$ & -1.220050 & 3.776865 & 2.4 \\
\hline $\mathrm{C}$ & -1.707163 & 4.618924 & 0.0 \\
\hline C & -0.187283 & 4.404611 & -1.8 \\
\hline C & -0.139797 & 2.855629 & -3.7 \\
\hline C & -0.061177 & 0.570474 & -4.6 \\
\hline $\mathrm{C}$ & -0.127885 & -1.859484 & $-4 \cdot 3$ \\
\hline $\mathrm{C}$ & 1.087089 & -3.818996 & $-2.6 \varepsilon$ \\
\hline $\mathrm{C}$ & 0.795817 & -4.738224 & -0.44 \\
\hline $\mathrm{C}$ & 0.483018 & -4.372027 & 1.94 \\
\hline $\mathrm{C}$ & 0.223327 & -2.820766 & 3.81 \\
\hline $\mathrm{C}$ & 0.078725 & -0.520733 & 4.6 \\
\hline $\mathrm{C}$ & 0.085321 & 1.879038 & 4.1 \\
\hline $\mathrm{C}$ & 0.240317 & 3.764157 & 2.5 \\
\hline $\mathrm{C}$ & 0.776118 & 4.758613 & 0.27 \\
\hline $\mathrm{C}$ & 1.212799 & 4.499183 & -1.9 \\
\hline $\mathrm{C}$ & 1.303530 & 2.859469 & -3.7 \\
\hline $\mathrm{C}$ & 1.361785 & 0.549000 & -4.45 \\
\hline $\mathrm{C}$ & 1.289323 & -1.875925 & -4.16 \\
\hline $\mathrm{C}$ & 1.905635 & -2.952629 & -3.467 \\
\hline $\mathrm{C}$ & 1.653125 & -4.428934 & -1.5 \\
\hline $\mathrm{C}$ & 1.342655 & -4.707488 & 0.86 \\
\hline $\mathrm{C}$ & 1.047567 & -3.706987 & 3.07 \\
\hline $\mathrm{C}$ & 0.836255 & -1.707087 & 4.45 \\
\hline $\mathrm{C}$ & 0.760803 & 0.726331 & 4.615 \\
\hline $\mathrm{C}$ & 0.846452 & 2.929700 & 3.5 \\
\hline $\mathrm{C}$ & 1.137803 & 4.407083 & 1.5997 \\
\hline $\mathrm{C}$ & 1.764897 & 4.847077 & -0.716 \\
\hline $\mathrm{C}$ & 1.992656 & 3.803536 & -2.8987 \\
\hline $\mathrm{C}$ & 2.055912 & 1.742492 & -4.1765 \\
\hline $\mathrm{C}$ & 2.045936 & -0.698697 & -4 \\
\hline $\mathrm{C}$ & 3.322576 & -2.967905 & -3.300 \\
\hline $\mathrm{C}$ & 3.07 & -4 & -1 \\
\hline
\end{tabular}




\begin{tabular}{|c|c|c|c|}
\hline C & 2.763830 & -4.687085 & 1.04912 \\
\hline C & 2.468087 & -3.674279 & 3.2505 \\
\hline C & 2.252260 & -1.676061 & 4.63191 \\
\hline $\mathrm{C}$ & 2.169783 & 0.752572 & 4.82131 \\
\hline $\mathrm{C}$ & 2.260036 & 2.970845 & 3.8014 \\
\hline C & 2.578513 & 4.438379 & 1.8787 \\
\hline C & 3.135016 & 4.784742 & -0.4788 \\
\hline C & 3.413387 & 3.754282 & -2.6874 \\
\hline $\mathrm{C}$ & 3.490520 & 1.716924 & -4.0340 \\
\hline C & 3.465750 & -0.724261 & -4.2604 \\
\hline $\mathrm{C}$ & 4.108127 & -1.921811 & -3.8486 \\
\hline $\mathrm{C}$ & 3.909245 & -3.835830 & -2.3379 \\
\hline $\mathrm{C}$ & 3.624914 & -4.713902 & -0.0781 \\
\hline $\mathrm{C}$ & 3.316025 & -4.313745 & 2.30662 \\
\hline $\mathrm{C}$ & 3.050851 & -2.752437 & 4.1605 \\
\hline $\mathrm{C}$ & 2.889165 & -0.460471 & 4.9858 \\
\hline $\mathrm{C}$ & 2.879868 & 1.950451 & 4.5767 \\
\hline C & 3.089548 & 3.853599 & 3.067 \\
\hline $\mathrm{C}$ & 3.550198 & 4.745107 & 0.8644 \\
\hline $\mathrm{C}$ & 3.995053 & 4.372890 & -1.5368 \\
\hline $\mathrm{C}$ & 4.178281 & 2.792330 & -3.38661 \\
\hline $\mathrm{C}$ & 4.193718 & 0.499076 & -4.220 \\
\hline $\mathrm{C}$ & 5.542041 & -1.952787 & -3.6903 \\
\hline $\mathrm{C}$ & 5.342151 & -3.856530 & -2.159 \\
\hline $\mathrm{C}$ & 5.057940 & -4.723340 & 0.11 \\
\hline $\mathrm{C}$ & 4.748690 & -4.307523 & 2.5017 \\
\hline $\mathrm{C}$ & 4.482905 & -2.732924 & 4.35 \\
\hline $\mathrm{C}$ & 4.315160 & -0.438362 & 5.2065 \\
\hline $\mathrm{C}$ & 4.295264 & 1.987740 & 4.8539 \\
\hline C & 4.511453 & 3.902508 & 3.35 \\
\hline $\mathrm{C}$ & 4.975360 & 4.715127 & 1.1073 \\
\hline $\mathrm{C}$ & 5.408262 & 4.284601 & -1.2812 \\
\hline $\mathrm{C}$ & 5.609303 & 2.743863 & -3.1750 \\
\hline $\mathrm{C}$ & 5.632123 & 0.467337 & -4.0655 \\
\hline $\mathrm{C}$ & 5.880190 & -4.459620 & -1.0061 \\
\hline $\mathrm{C}$ & 5.571056 & -4.698606 & 1.4237 \\
\hline $\mathrm{C}$ & 5.272386 & -3.651947 & $3.6370^{\prime}$ \\
\hline $\mathrm{C}$ & 5.048231 & -1.631548 & 5.0387 \\
\hline C & 4.945388 & 0.819300 & 5.3067 \\
\hline $\mathrm{C}$ & 5.037865 & 3.078499 & 4.3695 \\
\hline $\mathrm{C}$ & 5.399470 & 4.480376 & 2.4269 \\
\hline $\mathrm{C}$ & 5.866113 & 4.642120 & 0.0120 \\
\hline $\mathrm{C}$ & 6.204226 & 3.609247 & -2.2304 \\
\hline $\mathrm{C}$ & 6.315998 & 1.640832 & -3.6993 \\
\hline $\mathrm{H}$ & -6.063774 & 1.872568 & -5.1043 \\
\hline $\mathrm{H}$ & -7.085970 & -3.818651 & 2.06556 \\
\hline $\mathrm{H}$ & -6.789939 & -4.759613 & -0.1890 \\
\hline
\end{tabular}




$\begin{array}{rrrr}\mathrm{H} & -6.473721 & -4.406133 & -2.597220 \\ \mathrm{H} & -7.288204 & -1.870530 & 3.564170 \\ \mathrm{H} & -7.371637 & 0.546903 & 3.933340 \\ \mathrm{H} & -7.386303 & 2.757126 & 3.033992 \\ \mathrm{H} & 6.349293 & -3.626212 & 3.768005 \\ \mathrm{H} & 6.647541 & -4.677694 & 1.559153 \\ \mathrm{H} & 6.956979 & -4.453116 & -0.868446 \\ \mathrm{H} & 7.390567 & 1.592639 & -3.552559 \\ \mathrm{H} & 6.123789 & -1.606691 & 5.182874 \\ \mathrm{H} & 7.272346 & 3.526698 & -2.057107 \\ \mathrm{H} & 6.016599 & 0.856268 & 5.480179 \\ \mathrm{H} & 6.929647 & 4.582879 & 0.219887 \\ \mathrm{H} & 6.105669 & 3.113823 & 4.563593 \\ \mathrm{H} & 6.462097 & 4.472756 & 2.649826 \\ \mathrm{C} & -6.185743 & 4.335830 & 1.178192 \\ \mathrm{H} & -7.257008 & 4.235812 & 1.037509 \\ \mathrm{C} & -5.715322 & 4.669104 & -1.296554 \\ \mathrm{H} & -6.758597 & 4.597353 & -1.585160 \\ \mathrm{C} & -5.195388 & 3.793339 & -3.494194 \\ \mathrm{H} & -6.258742 & 3.823336 & -3.708739 \\ \mathrm{C} & -4.997176 & -0.600872 & -5.246525 \\ \mathrm{H} & -6.073871 & -0.584719 & -5.387051 \\ \mathrm{C} & -5.139330 & -2.884948 & -4.359031 \\ \mathrm{H} & -6.217374 & -2.866845 & -4.487499 \\ \mathrm{C} & 6.277524 & -0.784651 & -3.966952 \\ \mathrm{H} & 7.355661 & -0.802902 & -3.837544 \\ \mathrm{C} & 6.130119 & -3.017673 & -2.976527 \\ \mathrm{H} & 7.208072 & -3.024641 & -2.844873\end{array}$

Nanotube-O (Singlet) $Z P E=1.187321 \quad \mathrm{Ee}=-5576.585329$

$\begin{array}{llrl}\text { C } & -5.864601 & -3.689359 & -2.854450 \\ \text { C } & -5.182715 & -2.798523 & -3.707401 \\ \text { C } & -5.856355 & -1.694680 & -4.271546 \\ \text { C } & -5.167455 & -0.492751 & -4.532500 \\ \text { C } & -5.830572 & 0.749805 & -4.475403 \\ \text { C } & -5.136804 & 1.916620 & -4.089882 \\ \text { C } & -5.786852 & 2.986924 & -3.441311 \\ \text { C } & -5.077659 & 3.820533 & -2.551564 \\ \text { C } & -5.688031 & 4.481862 & -1.462743 \\ \text { C } & -4.949594 & 4.805037 & -0.304396 \\ \text { C } & -5.552630 & 4.866180 & 0.969805 \\ \text { C } & -4.807918 & 4.512642 & 2.112686 \\ \text { C } & -5.443287 & 3.889276 & 3.203314 \\ \text { C } & -4.747536 & 2.939127 & 3.978397 \\ \text { C } & -5.444325 & 1.868145 & 4.571643 \\ \text { C } & -4.812261 & 0.622973 & 4.775532\end{array}$




\begin{tabular}{|c|c|c|c|}
\hline $\mathrm{C}$ & -5.544793 & -0.581050 & 4.76999 \\
\hline C & -4.937166 & -1.791948 & 4.37120 \\
\hline C & -5.673779 & -2.841366 & 3.78434 \\
\hline $\mathrm{C}$ & -5.059113 & -3.724391 & 2.8715 \\
\hline C & -5.781411 & -4.346034 & 1.8302 \\
\hline C & -5.142665 & -4.672689 & 0.6169 \\
\hline $\mathrm{C}$ & -5.844507 & -4.666386 & -0.607 \\
\hline $\mathrm{C}$ & -5.179486 & -4.342653 & -1.8079 \\
\hline $\mathrm{C}$ & -3.740069 & -2.822371 & -3.72893 \\
\hline C & -3.027558 & -1.734889 & -4.2986 \\
\hline C & -3.722370 & -0.519710 & -4.546 \\
\hline C & -2.999422 & 0.699012 & -4.499 \\
\hline $\mathrm{C}$ & -3.689531 & 1.884660 & -4.11 \\
\hline C & -2.952448 & 2.928201 & -3.50 \\
\hline $\mathrm{C}$ & -3.636124 & 3.786749 & -2.59 \\
\hline C & -2.883547 & 4.413397 & -1.574 \\
\hline $\mathrm{C}$ & -3.507099 & 4.783747 & -0.36 \\
\hline C & -2.713001 & 4.820037 & 0.83 \\
\hline $\mathrm{C}$ & -3.363445 & 4.471199 & $2.03^{\circ}$ \\
\hline C & -2.612377 & 3.775857 & 3.01 \\
\hline C & -3.308181 & 2.877802 & 3.87 \\
\hline $\mathrm{C}$ & -2.628239 & 1.744753 & 4.37 \\
\hline C & -3.372576 & 0.571370 & 4.68 \\
\hline $\mathrm{C}$ & -2.724469 & -0.685833 & 4.62 \\
\hline C & -3.494161 & -1.840734 & 4.31 \\
\hline C & -2.847920 & -2.935672 & 3.67 \\
\hline $\mathrm{C}$ & -3.614666 & -3.772960 & 2.82 \\
\hline C & -2.952983 & -4.426396 & 1.74 \\
\hline C & -3.698319 & -4.713749 & 0.57 \\
\hline C & -3.015878 & -4.729604 & -0.6 \\
\hline $\mathrm{C}$ & -3.736618 & -4.372817 & -1.84 \\
\hline C & -3.036642 & -3.736672 & -2.90 \\
\hline C & -1.598363 & -1.751845 & -4.31 \\
\hline C & -0.877000 & -0.554442 & -4.582 \\
\hline C & -1.569235 & 0.681992 & -4.5 \\
\hline $\mathrm{C}$ & -0.836888 & 1.857055 & -4.1828 \\
\hline C & -1.518884 & 2.922056 & -3.548 \\
\hline $\mathrm{C}$ & -0.773588 & 3.815931 & -2.727 \\
\hline C & -1.464341 & 4.444691 & -1.6585 \\
\hline C & -0.721271 & 4.947488 & -0.57 \\
\hline C & -1.278372 & 4.882572 & 0.7620 \\
\hline $\mathrm{C}$ & -0.497589 & 4.480627 & 1.905 \\
\hline C & -1.182026 & 3.734160 & 2.9241 \\
\hline C & -0.477711 & 2.748496 & 3.6888 \\
\hline C & -1.203321 & 1.684009 & 4.28 \\
\hline C & -0.537941 & 0.451178 & 4.553 \\
\hline$C$ & -1.296646 & -0.746356 & 4 \\
\hline
\end{tabular}




\begin{tabular}{|c|c|c|c|}
\hline $\mathrm{C}$ & -0.649976 & -1.970893 & 4.24653 \\
\hline $\mathrm{C}$ & -1.418734 & -3.005589 & 3.65194 \\
\hline C & -0.767179 & -3.914915 & 2.77501 \\
\hline $\mathrm{C}$ & -1.523528 & -4.492554 & 1.71907 \\
\hline C & -0.851816 & -4.828296 & 0.51248 \\
\hline $\mathrm{C}$ & -1.586180 & -4.774123 & -0.7049 \\
\hline $\mathrm{C}$ & -0.891737 & -4.437117 & -1.898 \\
\hline $\mathrm{C}$ & -1.606950 & -3.760221 & -2.925 \\
\hline C & -0.895916 & -2.858809 & -3.7650 \\
\hline $\mathrm{C}$ & 0.552704 & -0.572792 & -4.597 \\
\hline $\mathrm{C}$ & 1.277207 & 0.647454 & -4.5584 \\
\hline $\mathrm{C}$ & 0.591214 & 1.839205 & -4.198 \\
\hline C & 1.326549 & 2.895527 & -3.5967 \\
\hline C & 0.654101 & 3.810013 & -2.7544 \\
\hline $\mathrm{C}$ & 1.396097 & 4.587596 & -1.80 \\
\hline $\mathrm{C}$ & 0.631267 & 5.210584 & -0.817 \\
\hline $\mathrm{C}$ & 1.763359 & 5.119612 & 1.01 \\
\hline $\mathrm{C}$ & 0.964678 & 4.458293 & 1.94 \\
\hline C & 1.670898 & 3.588400 & 2.84 \\
\hline C & 0.951274 & 2.666959 & 3.6 \\
\hline C & 1.632284 & 1.551156 & 4.1 \\
\hline $\mathrm{C}$ & 0.885376 & 0.386187 & 4.51 \\
\hline C & 1.539515 & -0.873138 & 4.5 \\
\hline $\mathrm{C}$ & 0.774288 & -2.035809 & 4.21 \\
\hline $\mathrm{C}$ & 1.422446 & -3.140900 & 3.60 \\
\hline C & 0.658866 & -3.980922 & 2.74 \\
\hline $\mathrm{C}$ & 1.320206 & -4.618591 & 1.65 \\
\hline C & 0.575448 & -4.881110 & 0.47 \\
\hline $\mathrm{C}$ & 1.259074 & -4.860665 & -0.770 \\
\hline C & 0.536674 & -4.468109 & -1.9283 \\
\hline $\mathrm{C}$ & 1.239887 & -3.807850 & -2.97 \\
\hline C & 0.533468 & -2.877701 & -3.7836 \\
\hline C & 1.249317 & -1.786406 & $-4 \cdot 3$ \\
\hline $\mathrm{C}$ & 2.705168 & 0.637153 & -4.5785 \\
\hline $\mathrm{C}$ & 3.427394 & 1.828307 & -4.270 \\
\hline $\mathrm{C}$ & 2.758233 & 2.905031 & -3.63 \\
\hline $\mathrm{C}$ & 3.494193 & 3.831585 & -2.8349 \\
\hline $\mathrm{C}$ & 2.856727 & 4.538780 & -1.7656 \\
\hline C & 3.646679 & 4.844882 & -0.6010 \\
\hline C & 3.104180 & 4.808749 & 0.7413 \\
\hline $\mathrm{C}$ & 3.825434 & 4.190456 & 1.778 \\
\hline C & 3.102223 & 3.518753 & 2.8027 \\
\hline $\mathrm{C}$ & 3.797970 & 2.542164 & 3.570 \\
\hline C & 3.061022 & 1.486550 & 4.1612 \\
\hline $\mathrm{C}$ & 3.723648 & 0.260430 & 4.458 \\
\hline C & 2.968137 & -0.938180 & 4.480 \\
\hline $\mathrm{C}$ & 3.612889 & -2.168649 & 4 \\
\hline
\end{tabular}




\begin{tabular}{|c|c|c|c|}
\hline $\mathrm{C}$ & 2.852046 & -3.206601 & 3.57842 \\
\hline $\mathrm{C}$ & 3.498685 & -4.117433 & 2.70141 \\
\hline $\mathrm{C}$ & 2.750418 & -4.677353 & 1.6313 \\
\hline $\mathrm{C}$ & 3.417219 & -4.989702 & 0.41769 \\
\hline C & 2.689620 & -4.898856 & -0.80034 \\
\hline $\mathrm{C}$ & 3.381481 & -4.527727 & -1.9838 \\
\hline $\mathrm{C}$ & 2.670247 & -3.826015 & -2.995 \\
\hline $\mathrm{C}$ & 3.379592 & -2.908135 & -3.8176 \\
\hline $\mathrm{C}$ & 2.678488 & -1.798297 & -4.3605 \\
\hline $\mathrm{C}$ & 3.395516 & -0.598897 & -4.632 \\
\hline $\mathrm{C}$ & 4.868230 & 1.799144 & -4.333 \\
\hline $\mathrm{C}$ & 5.580269 & 2.873433 & -3.7 \\
\hline C & 4.932187 & 3.787572 & -2.913 \\
\hline $\mathrm{C}$ & 5.083091 & 4.706861 & -0.67 \\
\hline $\mathrm{C}$ & 5.260700 & 4.080458 & 1.68 \\
\hline $\mathrm{C}$ & 5.952093 & 3.344725 & 2.663 \\
\hline $\mathrm{C}$ & 5.245897 & 2.469497 & 3.51 \\
\hline C & 5.893498 & 1.365475 & 4.10 \\
\hline C & 5.165972 & 0.201636 & 4.42 \\
\hline C & 5.795820 & -1.057758 & 4.42 \\
\hline $\mathrm{C}$ & 5.052743 & -2.222773 & 4.14 \\
\hline $\mathrm{C}$ & 5.679943 & -3.317549 & 3.5 \\
\hline $\mathrm{C}$ & 4.937838 & -4.163827 & 2.66 \\
\hline $\mathrm{C}$ & 5.580162 & -4.770227 & 1.56 \\
\hline $\mathrm{C}$ & 4.858479 & -5.025827 & $0.3^{\circ}$ \\
\hline $\mathrm{C}$ & 5.521868 & -4.971759 & -0.86 \\
\hline $\mathrm{C}$ & 4.826016 & -4.552684 & -2.01 \\
\hline $\mathrm{C}$ & 5.504835 & -3.877901 & -3.05 \\
\hline $\mathrm{C}$ & 4.826308 & -2.928966 & -3.84 \\
\hline $\mathrm{C}$ & 5.513539 & -1.837678 & -4.4 \\
\hline $\mathrm{C}$ & 4.842035 & -0.625496 & -4.66 \\
\hline $\mathrm{C}$ & 5.865861 & 4.522799 & 0.4 \\
\hline $\mathrm{H}$ & 6.945586 & 4.464885 & 0.35 \\
\hline $\mathrm{C}$ & 5.698165 & 4.378314 & -1.892 \\
\hline $\mathrm{H}$ & 6.782018 & 4.325735 & $-1 \cdot 9$ \\
\hline $\mathrm{C}$ & 5.535691 & 0.603166 & -4.66 \\
\hline $\mathrm{H}$ & 6.621310 & 0.596039 & -4.69 \\
\hline $\mathrm{H}$ & -6.871210 & 2.988494 & -3.40 \\
\hline $\mathrm{H}$ & -6.771315 & 4.482139 & -1.4000 \\
\hline $\mathrm{H}$ & -6.635939 & 4.878843 & 1.0 \\
\hline $\mathrm{H}$ & -6.526930 & 3.922152 & 3.2659 \\
\hline $\mathrm{H}$ & -6.915530 & 0.758010 & -4.44 \\
\hline $\mathrm{H}$ & -6.527752 & 1.918593 & 4.625 \\
\hline $\mathrm{H}$ & -6.941751 & -1.676888 & -4.2430 \\
\hline $\mathrm{H}$ & -6.62 & -0.530893 & 4.8 \\
\hline $\mathrm{H}$ & -6.949823 & -3.657432 & -2.826 \\
\hline $\mathrm{H}$ & -6 . & 0015 & 3 \\
\hline
\end{tabular}




$\begin{array}{rrrr}\mathrm{H} & -6.929060 & -4.622625 & -0.585568 \\ \mathrm{H} & -6.865259 & -4.294824 & 1.848639 \\ \mathrm{H} & 6.665885 & -4.792484 & 1.533335 \\ \mathrm{H} & 6.607622 & -4.985866 & -0.883361 \\ \mathrm{H} & 6.765064 & -3.352765 & 3.476342 \\ \mathrm{H} & 6.590384 & -3.888120 & -3.063559 \\ \mathrm{H} & 6.880182 & -1.102873 & 4.392362 \\ \mathrm{H} & 6.599040 & -1.849014 & -4.429498 \\ \mathrm{H} & 6.976672 & 1.309757 & 4.054380 \\ \mathrm{H} & 7.033130 & 3.265865 & 2.606894 \\ \mathrm{H} & 6.666194 & 2.864343 & -3.803280 \\ \mathrm{O} & 1.215700 & 6.061828 & 0.129191\end{array}$

$\begin{array}{cccc}\text { Nanotube-O (Triplet) ZPE }= & 1.185847 & \mathrm{E}= & -5576.678988 \\ \mathrm{C} & -5.877317 & -3.717002 & -2.760575 \\ \text { C } & -5.192906 & -2.866679 & -3.636577 \\ \text { C } & -5.868033 & -1.765173 & -4.245999 \\ \text { C } & -5.170216 & -0.580609 & -4.521716 \\ \text { C } & -5.829241 & 0.669209 & -4.504070 \\ \text { C } & -5.116840 & 1.846055 & -4.135098 \\ \text { C } & -5.766591 & 2.902179 & -3.488362 \\ \text { C } & -5.040840 & 3.779953 & -2.636370 \\ \text { C } & -5.660717 & 4.432398 & -1.548393 \\ \text { C } & -4.918686 & 4.816922 & -0.418972 \\ \text { C } & -5.538059 & 4.901351 & 0.863539 \\ \text { C } & -4.798124 & 4.572762 & 2.005357 \\ \text { C } & -5.438467 & 3.991760 & 3.126809 \\ \text { C } & -4.744516 & 3.038986 & 3.907492 \\ \text { C } & -5.443205 & 1.984446 & 4.524539 \\ \text { C } & -4.804601 & 0.743531 & 4.759327 \\ \text { C } & -5.543588 & -0.462675 & 4.767369 \\ \text { C } & -4.928150 & -1.680474 & 4.417628 \\ \text { C } & -5.675372 & -2.741083 & 3.844546 \\ \text { C } & -5.056391 & -3.652773 & 2.959680 \\ \text { C } & -5.788720 & -4.285332 & 1.938819 \\ \text { C } & -5.146065 & -4.647802 & 0.718046 \\ \text { C } & -5.854908 & -4.643781 & -0.487726 \\ \text { C } & -5.186701 & -4.362228 & -1.708233 \\ \text { C } & -3.756919 & -2.894208 & -3.660070 \\ \text { C } & -3.034919 & -1.815098 & -4.264650 \\ \text { C } & -3.720950 & -0.607103 & -4.535197 \\ \text { C } & -2.991612 & 0.611802 & -4.527985 \\ \text { C } & -3.672604 & 1.817031 & -4.171937 \\ \text { C } & -2.935159 & 2.863766 & -3.580804 \\ \text { C } & -3.614134 & 3.760958 & -2.698709 \\ \text { C } & -2.863981 & 4.395729 & -1.681612 \\ \text { C } & -3.487191 & 4.806180 & -0.483968 \\ & -5309 & \end{array}$




\begin{tabular}{|c|c|c|c|}
\hline $\mathrm{C}$ & -2.694792 & 4.858384 & 0.71831 \\
\hline $\mathrm{C}$ & -3.351234 & 4.524199 & 1.9271 \\
\hline $\mathrm{C}$ & -2.603200 & 3.852562 & 2.9264 \\
\hline $\mathrm{C}$ & -3.307812 & 2.966910 & 3.805 \\
\hline C & -2.629213 & 1.848771 & 4.3324 \\
\hline C & -3.374864 & 0.682345 & 4.6812 \\
\hline $\mathrm{C}$ & -2.726730 & -0.582079 & 4.6387 \\
\hline $\mathrm{C}$ & -3.492109 & -1.741673 & 4.3692 \\
\hline $\mathrm{C}$ & -2.848096 & -2.859395 & 3.7532 \\
\hline C & -3.611800 & -3.714228 & 2.9216 \\
\hline C & -2.956703 & -4.392451 & 1.857 \\
\hline C & -3.706401 & -4.702663 & 0.682 \\
\hline $\mathrm{C}$ & -3.030397 & -4.738037 & -0.5596 \\
\hline $\mathrm{C}$ & -3.757813 & -4.405740 & -1.7 \\
\hline C & -3.055106 & -3.785708 & -2.81 \\
\hline C & -1.610173 & -1.843252 & -4.285 \\
\hline $\mathrm{C}$ & -0.883312 & -0.659548 & -4.5 \\
\hline C & -1.568566 & 0.585310 & -4.54 \\
\hline C & -0.827485 & 1.764490 & -4.226 \\
\hline C & -1.502539 & 2.846260 & -3.6 \\
\hline C & -0.752328 & 3.754946 & -2.81 \\
\hline $\mathrm{C}$ & -1.441694 & 4.412313 & -1.7 \\
\hline C & -0.701483 & 4.939689 & -0.687 \\
\hline $\mathrm{C}$ & -1.264903 & 4.913804 & 0.654 \\
\hline C & -0.486895 & 4.528402 & 1.80 \\
\hline C & -1.176646 & 3.811538 & 2.84 \\
\hline $\mathrm{C}$ & -0.474972 & 2.836653 & 3.63 \\
\hline C & -1.202292 & 1.787287 & 4.24 \\
\hline C & -0.537818 & 0.559548 & 4.54 \\
\hline C & -1.297941 & -0.642695 & 4.5 \\
\hline $\mathrm{C}$ & -0.652945 & -1.868771 & 4.29 \\
\hline C & -1.425090 & -2.926889 & 3.72 \\
\hline C & -0.775470 & -3.846819 & 2.860 \\
\hline C & -1.535664 & -4.457159 & 1.824 \\
\hline C & -0.864867 & -4.814240 & 0.6 \\
\hline $\mathrm{C}$ & -1.600465 & -4.787321 & -0.5952 \\
\hline C & -0.906928 & -4.481215 & -1.805 \\
\hline $\mathrm{C}$ & -1.621830 & -3.819368 & -2.843 \\
\hline C & -0.910837 & -2.947470 & -3.7058 \\
\hline C & 0.547974 & -0.684888 & -4.5 \\
\hline C & 1.281053 & 0.532978 & -4.5773 \\
\hline $\mathrm{C}$ & 0.600907 & 1.737846 & -4.23 \\
\hline C & 1.342012 & 2.795182 & -3.65 \\
\hline $\mathrm{C}$ & 0.671870 & 3.738499 & -2.8335 \\
\hline $\mathrm{C}$ & 1.416059 & 4.529744 & -1.8 \\
\hline C & 0.650895 & 5.188607 & -0.93 \\
\hline $\mathrm{C}$ & 1.783566 & 5.138079 & 0 \\
\hline
\end{tabular}




\begin{tabular}{|c|c|c|c|}
\hline $\mathrm{C}$ & 0.977520 & 4.505138 & 1.852043 \\
\hline $\mathrm{C}$ & 1.678937 & 3.661060 & 2.773850 \\
\hline $\mathrm{C}$ & 0.951440 & 2.752705 & 3.58993 \\
\hline $\mathrm{C}$ & 1.628311 & 1.649584 & 4.16495 \\
\hline C & 0.878487 & 0.491279 & 4.51513 \\
\hline $\mathrm{C}$ & 1.533019 & -0.773028 & 4.5280 \\
\hline $\mathrm{C}$ & 0.769204 & -1.935411 & 4.2655 \\
\hline $\mathrm{C}$ & 1.419058 & -3.059141 & 3.6672 \\
\hline C & 0.654096 & -3.912225 & 2.830 \\
\hline $\mathrm{C}$ & 1.313233 & -4.577638 & 1.7600 \\
\hline $\mathrm{C}$ & 0.562135 & -4.868180 & 0.5798 \\
\hline $\mathrm{C}$ & 1.241051 & -4.877768 & -0.663 \\
\hline C & 0.513879 & -4.519049 & -1.837 \\
\hline C & 1.218195 & -3.882366 & -2.899 \\
\hline $\mathrm{C}$ & 0.514392 & -2.975188 & -3.72 \\
\hline $\mathrm{C}$ & 1.238006 & -1.894332 & -4.3238 \\
\hline $\mathrm{C}$ & 2.702428 & 0.512200 & -4.59 \\
\hline $\mathrm{C}$ & 3.437400 & 1.702482 & -4.30 \\
\hline C & 2.772149 & 2.791833 & -3.691 \\
\hline C & 3.519939 & 3.728367 & -2.90 \\
\hline C & 2.877132 & 4.469255 & -1.852 \\
\hline $\mathrm{C}$ & 3.670837 & 4.796296 & -0.697 \\
\hline C & 3.119875 & 4.816292 & 0.65 \\
\hline $\mathrm{C}$ & 3.833779 & 4.218976 & 1.703 \\
\hline $\mathrm{C}$ & 3.104496 & 3.588002 & 2.744 \\
\hline C & 3.797540 & 2.625865 & 3.54 \\
\hline $\mathrm{C}$ & 3.059316 & 1.585456 & 4.146 \\
\hline C & 3.722795 & 0.365049 & 4.47 \\
\hline $\mathrm{C}$ & 2.963067 & -0.839048 & 4.50 \\
\hline $\mathrm{C}$ & 3.609529 & -2.062940 & 4.217 \\
\hline $\mathrm{C}$ & 2.841416 & -3.122585 & 3.63 \\
\hline C & 3.490118 & -4.033258 & 2.769 \\
\hline C & 2.735035 & -4.634127 & 1.72 \\
\hline $\mathrm{C}$ & 3.404317 & -4.973469 & 0.510 \\
\hline C & 2.672249 & -4.921420 & -0.696 \\
\hline $\mathrm{C}$ & 3.365129 & -4.594143 & -1.902 \\
\hline $\mathrm{C}$ & 2.651221 & -3.910699 & -2.9274 \\
\hline $\mathrm{C}$ & 3.360460 & -3.027448 & -3.7766 \\
\hline C & 2.662057 & -1.916380 & -4.3432 \\
\hline C & 3.385167 & -0.731506 & -4.6322 \\
\hline C & 4.875904 & 1.679232 & -4.3814 \\
\hline C & 5.588389 & 2.767632 & -3.8553 \\
\hline $\mathrm{C}$ & 4.953465 & 3.691954 & -2.9877 \\
\hline C & 5.105771 & 4.668309 & -0.7692 \\
\hline $\mathrm{C}$ & 5.271164 & 4.120868 & 1.620 \\
\hline C & 5.953902 & 3.425524 & 2.62 \\
\hline $\mathrm{C}$ & 5.237470 & 2.552891 & 3 \\
\hline
\end{tabular}




\begin{tabular}{|c|c|c|c|}
\hline $\mathrm{C}$ & 5.880124 & 1.458830 & 4.085199 \\
\hline $\mathrm{C}$ & 5.149686 & 0.300181 & 4.447985 \\
\hline $\mathrm{C}$ & 5.781571 & -0.967256 & 4.459184 \\
\hline C & 5.045916 & -2.128170 & 4.196020 \\
\hline $\mathrm{C}$ & 5.671585 & -3.267698 & 3.607679 \\
\hline$C$ & 4.937528 & -4.103533 & 2.745890 \\
\hline $\mathrm{C}$ & 5.567560 & -4.776624 & 1.680670 \\
\hline $\mathrm{C}$ & 4.843721 & -5.028968 & 0.476420 \\
\hline C & 5.496520 & -5.010200 & -0.759109 \\
\hline $\mathrm{C}$ & 4.794727 & -4.625834 & -1.932583 \\
\hline $\mathrm{C}$ & 5.474415 & -3.966186 & -2.982596 \\
\hline $\mathrm{C}$ & 4.797497 & -3.054121 & -3.802527 \\
\hline $\mathrm{C}$ & 5.492788 & -1.967859 & -4.414433 \\
\hline C & 4.830688 & -0.760920 & -4.675961 \\
\hline $\mathrm{C}$ & 5.880305 & 4.549676 & 0.422270 \\
\hline $\mathrm{H}$ & 6.960617 & 4.494975 & 0.330937 \\
\hline $\mathrm{C}$ & 5.715063 & 4.323783 & -1.987177 \\
\hline $\mathrm{H}$ & 6.797816 & 4.260411 & -2.034837 \\
\hline $\mathrm{C}$ & 5.529816 & 0.470808 & -4.72 \\
\hline $\mathrm{H}$ & 6.614555 & 0.457463 & -4.768525 \\
\hline $\mathrm{H}$ & -6.851500 & 2.907012 & -3.443291 \\
\hline $\mathrm{H}$ & -6.744572 & 4.421229 & -1.481798 \\
\hline $\mathrm{H}$ & -6.621811 & 4.923438 & 0.922252 \\
\hline $\mathrm{H}$ & -6.520681 & 4.041214 & 3.196917 \\
\hline $\mathrm{H}$ & -6.914610 & 0.690694 & -4.490499 \\
\hline $\mathrm{H}$ & -6.526538 & 2.034701 & 4.578566 \\
\hline $\mathrm{H}$ & -6.953477 & -1.747181 & -4.227797 \\
\hline $\mathrm{H}$ & -6.627793 & -0.411065 & 4.799502 \\
\hline $\mathrm{H}$ & -6.961570 & -3.671433 & -2.721715 \\
\hline $\mathrm{H}$ & -6.759710 & -2.692544 & 3.873721 \\
\hline $\mathrm{H}$ & -6.938896 & -4.584793 & -0.462984 \\
\hline $\mathrm{H}$ & -6.873337 & -4.241258 & 1.961617 \\
\hline $\mathrm{H}$ & 6.651720 & -4.828039 & 1.656548 \\
\hline $\mathrm{H}$ & 6.582070 & -5.022566 & -0.782659 \\
\hline $\mathrm{H}$ & 6.756068 & -3.316116 & 3.591997 \\
\hline $\mathrm{H}$ & 6.560330 & -3.972393 & -2.985559 \\
\hline $\mathrm{H}$ & 6.865906 & -1.008832 & 4.418117 \\
\hline $\mathrm{H}$ & 6.578168 & -1.991207 & -4.434887 \\
\hline $\mathrm{H}$ & 6.963209 & 1.397042 & 4.036507 \\
\hline $\mathrm{H}$ & 7.036787 & 3.358170 & 2.591812 \\
\hline $\mathrm{H}$ & 6.673433 & 2.756690 & -3.894386 \\
\hline 0 & 1.244336 & 6.055636 & -0.00726 \\
\hline
\end{tabular}

\begin{tabular}{|c|c|c|c|c|}
\hline \multicolumn{3}{|c|}{ SW Defected Nanotube-O (Singlet) ZPE= } & 1.184872 & -5576.502223 \\
\hline $\mathrm{C}$ & -5.665986 & -4.819143 & -1.025018 & \\
\hline $\mathrm{C}$ & -5.973630 & -4.400457 & 1.380050 & \\
\hline $\mathrm{C}$ & -6.203044 & -2.784952 & 3.222624 & \\
\hline
\end{tabular}




\begin{tabular}{|c|c|c|c|}
\hline $\mathrm{C}$ & -6.326962 & -0.462768 & 4.00065 \\
\hline $\mathrm{C}$ & -6.384518 & 1.919373 & 3.49891 \\
\hline $\mathrm{C}$ & -4.936261 & 2.785750 & -4.46198 \\
\hline $\mathrm{C}$ & -5.350800 & -3.908940 & -3.28536 \\
\hline C & -4.833469 & -4.522245 & -2.1208 \\
\hline C & -5.153901 & -4.760758 & 0.2889 \\
\hline $\mathrm{C}$ & -5.428079 & -3.699436 & 2.4732 \\
\hline $\mathrm{C}$ & -5.601992 & -1.661298 & 3.8206 \\
\hline $\mathrm{C}$ & -5.680558 & 0.787560 & 3.97350 \\
\hline C & -5.690959 & 2.985054 & 2.8924 \\
\hline C & -5.422567 & 4.433992 & 0.901 \\
\hline C & -4.708269 & 4.771091 & -1.4222 \\
\hline $\mathrm{C}$ & -4.340018 & 3.693664 & -3.562 \\
\hline $\mathrm{C}$ & -4.257658 & 1.618919 & -4.8508 \\
\hline $\mathrm{C}$ & -4.335496 & -0.823891 & -5.03 \\
\hline C & -4.540171 & -3.064237 & -4.063 \\
\hline $\mathrm{C}$ & -3.400501 & -4.546750 & -1.9 \\
\hline C & -3.720611 & -4.757719 & 0.46 \\
\hline C & -3.994350 & -3.673225 & 2.635 \\
\hline C & -4.166457 & -1.620153 & 3.9 \\
\hline C & -4.233460 & 0.823070 & 4.10 \\
\hline $\mathrm{C}$ & -4.261114 & 3.029010 & 3.1 \\
\hline C & -4.039454 & 4.481852 & 1.1 \\
\hline $\mathrm{C}$ & -3.261194 & 4.732522 & -1.11 \\
\hline C & -2.920642 & 3.615430 & -3.2 \\
\hline $\mathrm{C}$ & -2.830911 & 1.558613 & -4.62 \\
\hline $\mathrm{C}$ & -2.902704 & -0.878103 & -4.858 \\
\hline C & -3.106982 & -3.112315 & -3.8886 \\
\hline C & -2.543057 & -3.985725 & -2.923 \\
\hline C & -2.856486 & -4.835271 & -0.6 \\
\hline $\mathrm{C}$ & -3.162793 & -4.365743 & 1.7 \\
\hline $\mathrm{C}$ & -3.393529 & -2.716720 & 3.50 \\
\hline C & -3.511245 & -0.386430 & 4.22 \\
\hline C & -3.524671 & 2.014412 & 3.800 \\
\hline C & -3.508236 & 3.898645 & $2.3^{\circ}$ \\
\hline $\mathrm{C}$ & -2.993942 & 4.773622 & 0.291 \\
\hline $\mathrm{C}$ & -2.342315 & 4.344919 & -2.206 \\
\hline $\mathrm{C}$ & -2.157071 & 2.628941 & -4.00 \\
\hline C & -2.149734 & 0.319481 & -4.8098 \\
\hline C & -2.290173 & -2.086918 & -4.4 \\
\hline C & -1.123537 & -4.035801 & -2.7453 \\
\hline $\mathrm{C}$ & -1.436204 & -4.864731 & -0.47 \\
\hline C & -1.743041 & -4.368788 & 1.8 \\
\hline $\mathrm{C}$ & -1.974790 & -2.691167 & 3.65 \\
\hline C & -2.088071 & -0.339488 & 4.3 \\
\hline C & -2.078652 & 2.065693 & 3.8 \\
\hline 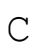 & -2.101323 & 3.918186 & \\
\hline
\end{tabular}




\begin{tabular}{|c|c|c|c|}
\hline C & -0.596328 & 5.145500 & 0.81070 \\
\hline C & -0.866628 & 4.414665 & -2.22597 \\
\hline C & -0.736954 & 2.571932 & $-3.8657 \varepsilon$ \\
\hline $\mathrm{C}$ & -0.735093 & 0.260746 & -4.65271 \\
\hline $\mathrm{C}$ & -0.872599 & -2.145307 & -4.2702 \\
\hline $\mathrm{C}$ & -0.283486 & -3.222653 & -3.5479 \\
\hline C & -0.572218 & -4.627839 & -1.57169 \\
\hline C & -0.890203 & -4.789382 & 0.8421 \\
\hline $\mathrm{C}$ & -1.168017 & -3.645464 & 2.977 \\
\hline C & -1.345532 & -1.544215 & 4.2050 \\
\hline $\mathrm{C}$ & -1.390623 & 0.902421 & 4.242 \\
\hline $\mathrm{C}$ & -1.312011 & 3.112279 & 3.1538 \\
\hline $\mathrm{C}$ & -1.780554 & 4.624485 & 1.1192 \\
\hline $\mathrm{C}$ & -0.104539 & 5.176180 & -1.35538 \\
\hline $\mathrm{C}$ & -0.079997 & 3.558401 & -3.0939 \\
\hline $\mathrm{C}$ & -0.013212 & 1.441098 & -4.3308 \\
\hline $\mathrm{C}$ & -0.084102 & -0.997042 & -4.53832 \\
\hline $\mathrm{C}$ & 1.133722 & -3.271064 & -3.3780 \\
\hline $\mathrm{C}$ & 0.844674 & -4.657439 & -1.3877 \\
\hline $\mathrm{C}$ & 0.526690 & -4.791419 & 1.0282 \\
\hline $\mathrm{C}$ & 0.246624 & -3.626735 & 3.1516 \\
\hline $\mathrm{C}$ & 0.066395 & -1.514959 & 4.35 \\
\hline $\mathrm{C}$ & 0.032871 & 0.934145 & 4.34 \\
\hline $\mathrm{C}$ & 0.165519 & 3.146532 & 3.20 \\
\hline $\mathrm{C}$ & 0.723471 & 4.821469 & $1.2 \varepsilon$ \\
\hline $\mathrm{C}$ & 1.252204 & 4.982722 & -1.1077 \\
\hline $\mathrm{C}$ & 1.353283 & 3.526080 & -3.0 \\
\hline $\mathrm{C}$ & 1.410092 & 1.390956 & -4.2137 \\
\hline $\mathrm{C}$ & 1.335015 & -1.051656 & -4.3863 \\
\hline $\mathrm{C}$ & 1.951614 & -2.255766 & -3.94 \\
\hline $\mathrm{C}$ & 1.701186 & -4.118784 & -2.3895 \\
\hline $\mathrm{C}$ & 1.390382 & -4.895800 & -0.0968 \\
\hline $\mathrm{C}$ & 1.083030 & -4.346845 & 2.25 \\
\hline $\mathrm{C}$ & 0.842608 & -2.637036 & 3.9812 \\
\hline $\mathrm{C}$ & 0.726582 & -0.274471 & 4.58 \\
\hline $\mathrm{C}$ & 0.776388 & 2.111488 & 3.9901 \\
\hline $\mathrm{C}$ & 1.059956 & 4.028432 & 2.45 \\
\hline $\mathrm{C}$ & 1.699005 & 5.037311 & 0.2729 \\
\hline C & 2.029916 & 4.285714 & -2.0336 \\
\hline $\mathrm{C}$ & 2.105398 & 2.492521 & -3.6651 \\
\hline $\mathrm{C}$ & 2.093110 & 0.147722 & -4.3777 \\
\hline $\mathrm{C}$ & 3.369297 & -2.310921 & -3.7915 \\
\hline C & 3.120011 & -4.154824 & -2.2123 \\
\hline $\mathrm{C}$ & 2.810465 & -4.900231 & 0.0898 \\
\hline $\mathrm{C}$ & 2.503534 & -4.324756 & 2.440 \\
\hline $\mathrm{C}$ & 2.260023 & -2.615137 & 4.1622 \\
\hline $\mathrm{C}$ & 2.134765 & -0.268116 & 4.810 \\
\hline
\end{tabular}




\begin{tabular}{|c|c|c|c|}
\hline $\mathrm{C}$ & 2.182387 & 2.116200 & 4.28351 \\
\hline $\mathrm{C}$ & 2.488350 & 3.978309 & 2.74469 \\
\hline $\mathrm{C}$ & 3.066768 & 4.816600 & 0.5254 \\
\hline $\mathrm{C}$ & 3.431895 & 4.151583 & -1.79257 \\
\hline C & 3.535071 & 2.424143 & -3.50528 \\
\hline $\mathrm{C}$ & 3.513070 & 0.087966 & -4.2374 \\
\hline $\mathrm{C}$ & 4.155749 & -1.168528 & -4.0948 \\
\hline $\mathrm{C}$ & 3.955471 & -3.367979 & -3.0442 \\
\hline $\mathrm{C}$ & 3.670953 & -4.699297 & -1.0183 \\
\hline $\mathrm{C}$ & 3.357524 & -4.767155 & 1.398 \\
\hline $\mathrm{C}$ & 3.075147 & -3.573456 & 3.505 \\
\hline $\mathrm{C}$ & 2.876791 & -1.479350 & 4.74 \\
\hline C & 2.818589 & 0.962977 & 4.834 \\
\hline $\mathrm{C}$ & 2.996383 & 3.153441 & 3.784 \\
\hline $\mathrm{C}$ & 3.465383 & 4.510254 & 1.83 \\
\hline $\mathrm{C}$ & 3.972607 & 4.577769 & -0.552 \\
\hline $\mathrm{C}$ & 4.212654 & 3.339947 & -2.6 \\
\hline C & 4.242791 & 1.272464 & -3.92 \\
\hline C & 5.590850 & -1.226823 & -3.9 \\
\hline C & 5.387722 & -3.419283 & -2.8 \\
\hline $\mathrm{C}$ & 5.103092 & -4.731112 & -0.82 \\
\hline $\mathrm{C}$ & 4.789809 & -4.773894 & 1.59 \\
\hline $\mathrm{C}$ & 4.506113 & -3.566543 & 3.70 \\
\hline $\mathrm{C}$ & 4.300180 & -1.479274 & 4.97 \\
\hline $\mathrm{C}$ & 4.228240 & 0.966285 & 5.14 \\
\hline $\mathrm{C}$ & 4.408787 & 3.171650 & 4.1 \\
\hline $\mathrm{C}$ & 4.886488 & 4.450039 & 2.1 \\
\hline $\mathrm{C}$ & 5.382025 & 4.450924 & -0.2 \\
\hline $\mathrm{C}$ & 5.638812 & 3.259842 & -2.42 \\
\hline $\mathrm{C}$ & 5.681491 & 1.215144 & -3.7 \\
\hline $\mathrm{C}$ & 5.927185 & -4.240946 & -1.86 \\
\hline $\mathrm{C}$ & 5.617246 & -4.945946 & 0.46 \\
\hline $\mathrm{C}$ & 5.307174 & -4.328524 & 2.8 \\
\hline $\mathrm{C}$ & 5.052474 & -2.606121 & 4.58 \\
\hline $\mathrm{C}$ & 4.900629 & -0.255346 & 5.3 \\
\hline $\mathrm{C}$ & 4.944597 & 2.158835 & 4.93 \\
\hline $\mathrm{C}$ & 5.293538 & 3.961838 & 3.35 \\
\hline $\mathrm{C}$ & 5.808112 & 4.594670 & 1.04 \\
\hline $\mathrm{C}$ & 6.202634 & 3.948913 & -1.3282 \\
\hline $\mathrm{C}$ & 6.356173 & 2.289523 & -3.1 \\
\hline $\mathrm{H}$ & -6.005355 & 2.858171 & -4.6357 \\
\hline $\mathrm{H}$ & -7.049927 & -4.392582 & 1.2 \\
\hline $\mathrm{H}$ & -6.741777 & -4.788359 & -1.1646 \\
\hline $\mathrm{H}$ & -6.427856 & -3.865246 & -3.4141 \\
\hline $\mathrm{H}$ & -7.281837 & -2.796015 & 3.1 \\
\hline $\mathrm{H}$ & -7.406594 & -0.488958 & 3.8 \\
\hline $\mathrm{H}$ & -7 & 1 & \\
\hline
\end{tabular}




$\begin{array}{rrrr}\mathrm{H} & 6.383862 & -4.312031 & 2.966441 \\ \mathrm{H} & 6.693743 & -4.941878 & 0.605389 \\ \mathrm{H} & 7.003990 & -4.258172 & -1.725212 \\ \mathrm{H} & 7.428866 & 2.212970 & -3.011484 \\ \mathrm{H} & 6.127114 & -2.591631 & 4.740007 \\ \mathrm{H} & 7.266525 & 3.842336 & -1.144275 \\ \mathrm{H} & 5.968412 & -0.233459 & 5.542617 \\ \mathrm{H} & 6.866926 & 4.517970 & 1.270681 \\ \mathrm{H} & 6.006309 & 2.181571 & 5.157290 \\ \mathrm{H} & 6.351661 & 3.939320 & 3.600235 \\ \mathrm{C} & -6.251682 & 3.792032 & 1.868761 \\ \mathrm{H} & -7.317126 & 3.711318 & 1.678967 \\ \mathrm{C} & -5.725050 & 4.779405 & -0.423190 \\ \mathrm{H} & -6.754397 & 4.765045 & -0.764741 \\ \mathrm{C} & -5.143993 & 4.473525 & -2.727549 \\ \mathrm{H} & -6.205660 & 4.538091 & -2.941772 \\ \mathrm{C} & -4.948989 & 0.440073 & -5.185791 \\ \mathrm{H} & -6.024253 & 0.486932 & -5.331282 \\ \mathrm{C} & -5.096784 & -1.976667 & -4.777581 \\ \mathrm{H} & -6.174716 & -1.932764 & -4.902568 \\ \mathrm{C} & 6.326725 & -0.026246 & -3.952879 \\ \mathrm{H} & 7.403699 & -0.074650 & -3.823623 \\ \mathrm{C} & 6.177440 & -2.422648 & -3.484210 \\ \mathrm{H} & 7.254997 & -2.457546 & -3.353294 \\ \mathrm{O} & -0.688112 & 5.949759 & -0.347728\end{array}$

\section{SW Defected Nanotube-O (Triplet) ZPE $=1.184872 \quad \mathrm{Ee}=\quad-5576.607424$}

$\begin{array}{lrrr}\text { C } & -5.660890 & -4.817411 & -1.014004 \\ \text { C } & -5.973732 & -4.390013 & 1.380660 \\ \text { C } & -6.206212 & -2.768272 & 3.213182 \\ \text { C } & -6.330696 & -0.442899 & 3.991060 \\ \text { C } & -6.385065 & 1.941372 & 3.492964 \\ \text { C } & -4.925136 & 2.777281 & -4.506748 \\ \text { C } & -5.338358 & -3.914176 & -3.271728 \\ \text { C } & -4.816902 & -4.531407 & -2.123331 \\ \text { C } & -5.145598 & -4.761476 & 0.293085 \\ \text { C } & -5.425578 & -3.686064 & 2.478216 \\ \text { C } & -5.601577 & -1.640727 & 3.817057 \\ \text { C } & -5.677996 & 0.806911 & 3.967518 \\ \text { C } & -5.682334 & 3.007302 & 2.887505 \\ \text { C } & -5.403070 & 4.446554 & 0.888321 \\ \text { C } & -4.686129 & 4.766804 & -1.435235 \\ \text { C } & -4.328575 & 3.677098 & -3.574627 \\ \text { C } & -4.250924 & 1.596915 & -4.871572 \\ \text { C } & -4.320738 & -0.851885 & -5.050655 \\ \text { C } & -4.519452 & -3.082186 & -4.068804\end{array}$




\begin{tabular}{|c|c|c|c|}
\hline C & -3.390643 & -4.570593 & -1.93651 \\
\hline $\mathrm{C}$ & -3.714352 & -4.770911 & 0.48018 \\
\hline C & -3.997024 & -3.667669 & 2.64581 \\
\hline $\mathrm{C}$ & -4.174348 & -1.602603 & $3.9503^{\circ}$ \\
\hline $\mathrm{C}$ & -4.237742 & 0.843634 & 4.1004 \\
\hline C & -4.254899 & 3.060979 & 3.13250 \\
\hline C & -4.032482 & 4.525022 & 1.18657 \\
\hline C & -3.255282 & 4.755351 & -1.1295 \\
\hline $\mathrm{C}$ & -2.912158 & 3.610891 & -3.2918 \\
\hline C & -2.821130 & 1.539125 & -4.6314 \\
\hline $\mathrm{C}$ & -2.895774 & -0.902931 & -4.8638 \\
\hline $\mathrm{C}$ & -3.100382 & -3.137271 & -3.89454 \\
\hline $\mathrm{C}$ & -2.535648 & -4.007986 & -2.9153 \\
\hline $\mathrm{C}$ & -2.850933 & -4.854894 & $-0.6448 s$ \\
\hline $\mathrm{C}$ & -3.163306 & -4.370751 & 1.7266 \\
\hline $\mathrm{C}$ & -3.399038 & -2.706894 & 3.501 \\
\hline $\mathrm{C}$ & -3.516679 & -0.366094 & 4.221 \\
\hline $\mathrm{C}$ & -3.523190 & 2.042562 & 3.79 \\
\hline $\mathrm{C}$ & -3.500461 & 3.937689 & 2.376 \\
\hline $\mathrm{C}$ & -2.992093 & 4.794705 & 0.281 \\
\hline $\mathrm{C}$ & -2.337719 & 4.345949 & -2.22301 \\
\hline $\mathrm{C}$ & -2.147409 & 2.618778 & -4.01 \\
\hline $\mathrm{C}$ & -2.139256 & 0.307968 & -4.8147 \\
\hline $\mathrm{C}$ & -2.282439 & -2.102860 & -4.4324 \\
\hline $\mathrm{C}$ & -1.117146 & -4.052481 & -2.7345 \\
\hline $\mathrm{C}$ & -1.438183 & -4.879234 & -0.4572 \\
\hline $\mathrm{C}$ & -1.750418 & -4.371353 & 1.91 \\
\hline $\mathrm{C}$ & -1.982716 & -2.681295 & 3.6594 \\
\hline $\mathrm{C}$ & -2.092746 & -0.321752 & 4.324 \\
\hline C & -2.080744 & 2.088718 & 3.82 \\
\hline $\mathrm{C}$ & -2.101594 & 3.948873 & 2.3381 \\
\hline $\mathrm{C}$ & -0.594506 & 5.158657 & 0.79 \\
\hline $\mathrm{C}$ & -0.862914 & 4.414044 & -2.2425 \\
\hline $\mathrm{C}$ & -0.734175 & 2.563222 & -3.8776 \\
\hline $\mathrm{C}$ & -0.729311 & 0.249495 & -4.6565 \\
\hline $\mathrm{C}$ & -0.862415 & -2.158958 & -4.2638 \\
\hline $\mathrm{C}$ & -0.273409 & -3.237313 & -3.5425 \\
\hline $\mathrm{C}$ & -0.568580 & -4.638890 & -1.5629 \\
\hline C & -0.894524 & -4.793455 & 0.8557 \\
\hline $\mathrm{C}$ & -1.176937 & -3.637585 & 2.9900 \\
\hline $\mathrm{C}$ & -1.353325 & -1.529121 & 4.2109 \\
\hline $\mathrm{C}$ & -1.393107 & 0.918220 & 4.24 \\
\hline $\mathrm{C}$ & -1.311929 & 3.129341 & 3.1523 \\
\hline $\mathrm{C}$ & -1.777637 & 4.642233 & 1.1094 \\
\hline $\mathrm{C}$ & -0.100446 & 5.180034 & -1.3685 \\
\hline $\mathrm{C}$ & -0.077082 & 3.555997 & -3.1042 \\
\hline $\mathrm{C}$ & -0.009249 & 1.427777 & -4.334 \\
\hline
\end{tabular}




\begin{tabular}{|c|c|c|c|}
\hline C & -0.075088 & -1.014156 & -4.53642 \\
\hline $\mathrm{C}$ & 1.137222 & -3.284214 & -3.36932 \\
\hline C & 0.845910 & -4.667254 & $-1.3751 \mathrm{~s}$ \\
\hline $\mathrm{C}$ & 0.521747 & -4.796387 & 1.04462 \\
\hline $\mathrm{C}$ & 0.235535 & -3.622030 & 3.1674 \\
\hline C & 0.054033 & -1.501912 & 4.36592 \\
\hline $\mathrm{C}$ & 0.024400 & 0.949235 & 4.3480 \\
\hline C & 0.162328 & 3.159022 & 3.2060 \\
\hline $\mathrm{C}$ & 0.725529 & 4.832467 & 1.2745 \\
\hline C & 1.251541 & 4.986166 & -1.1198 \\
\hline C & 1.354447 & 3.522707 & -3.0311 \\
\hline $\mathrm{C}$ & 1.415999 & 1.378585 & -4.21782 \\
\hline $\mathrm{C}$ & 1.340150 & -1.067458 & -4.3823 \\
\hline $\mathrm{C}$ & 1.956431 & -2.266717 & -3.93791 \\
\hline $\mathrm{C}$ & 1.703641 & -4.131667 & -2.3730 \\
\hline $\mathrm{C}$ & 1.388148 & -4.909066 & -0.077 \\
\hline $\mathrm{C}$ & 1.074302 & -4.354347 & $2.2798^{\prime}$ \\
\hline $\mathrm{C}$ & 0.830848 & -2.634855 & 3.996 \\
\hline $\mathrm{C}$ & 0.717013 & -0.264486 & 4.591 \\
\hline $\mathrm{C}$ & 0.771674 & 2.124967 & 3.98 \\
\hline $\mathrm{C}$ & 1.060994 & 4.043890 & 2.4419 \\
\hline $\mathrm{C}$ & 1.701118 & 5.048062 & 0.26 \\
\hline $\mathrm{C}$ & 2.029597 & 4.283810 & -2.0465 \\
\hline $\mathrm{C}$ & 2.110377 & 2.487150 & -3.67800 \\
\hline $\mathrm{C}$ & 2.100218 & 0.138541 & -4.3806 \\
\hline $\mathrm{C}$ & 3.374287 & -2.319999 & -3.7762 \\
\hline $\mathrm{C}$ & 3.120535 & -4.166392 & $-2 \cdot 1$ \\
\hline $\mathrm{C}$ & 2.802339 & -4.914741 & 0.113 \\
\hline $\mathrm{C}$ & 2.488151 & -4.336235 & 2.4658 \\
\hline C & 2.242810 & -2.617559 & 4.18 \\
\hline $\mathrm{C}$ & 2.123100 & -0.262391 & 4.81 \\
\hline $\mathrm{C}$ & 2.178509 & 2.124928 & 4.27 \\
\hline $\mathrm{C}$ & 2.486986 & 3.988774 & 2.731 \\
\hline $\mathrm{C}$ & 3.066245 & 4.830309 & 0.509 \\
\hline $\mathrm{C}$ & 3.431274 & 4.159662 & -1.810 \\
\hline $\mathrm{C}$ & 3.534242 & 2.424007 & -3.5187 \\
\hline $\mathrm{C}$ & 3.515648 & 0.081592 & -4.2379 \\
\hline $\mathrm{C}$ & 4.162134 & -1.181442 & -4.0850 \\
\hline C & 3.961327 & -3.379515 & -3.0264 \\
\hline $\mathrm{C}$ & 3.667950 & -4.713868 & -0.9990 \\
\hline $\mathrm{C}$ & 3.343168 & -4.784979 & 1.4236 \\
\hline $\mathrm{C}$ & 3.054129 & -3.582892 & 3.5347 \\
\hline $\mathrm{C}$ & 2.858935 & -1.474499 & 4.7620 \\
\hline $\mathrm{C}$ & 2.811515 & 0.972548 & 4.8362 \\
\hline $\mathrm{C}$ & 2.997614 & 3.159340 & 3.7742 \\
\hline $\mathrm{C}$ & 3.463868 & 4.519199 & 1.8248 \\
\hline $\mathrm{C}$ & 3.969870 & 4.600914 & -0.572 \\
\hline
\end{tabular}




\begin{tabular}{|c|c|c|c|}
\hline $\mathrm{C}$ & 4.209106 & 3.351059 & -2.66277 \\
\hline $\mathrm{C}$ & 4.243198 & 1.266508 & -3.93426 \\
\hline $\mathrm{C}$ & 5.592042 & -1.242446 & -3.93510 \\
\hline $\mathrm{C}$ & 5.386681 & -3.429883 & -2.85651 \\
\hline C & 5.094148 & -4.737539 & -0.80945 \\
\hline $\mathrm{C}$ & 4.770696 & -4.776891 & 1.6176 \\
\hline $\mathrm{C}$ & 4.480606 & -3.562245 & 3.72861 \\
\hline $\mathrm{C}$ & 4.276759 & -1.467158 & 4.9927 \\
\hline $\mathrm{C}$ & 4.214661 & 0.979420 & 5.1 \\
\hline $\mathrm{C}$ & 4.405248 & 3.178174 & 4.1077 \\
\hline $\mathrm{C}$ & 4.880290 & 4.455140 & 2.0975 \\
\hline $\mathrm{C}$ & 5.367420 & 4.457230 & -0.30 \\
\hline C & 5.625533 & 3.254809 & -2.434 \\
\hline C & 5.677213 & 1.201188 & -3.766 \\
\hline $\mathrm{C}$ & 5.926524 & -4.249025 & -1.83 \\
\hline $\mathrm{C}$ & 5.609322 & -4.937458 & 0.4932 \\
\hline $\mathrm{C}$ & 5.293580 & -4.310192 & 2.8 \\
\hline C & 5.037193 & -2.592018 & 4.5 \\
\hline C & 4.888902 & -0.246144 & 5.34 \\
\hline C & 4.939141 & 2.169659 & 4.93 \\
\hline $\mathrm{C}$ & 5.294254 & 3.971002 & 3.3 \\
\hline $\mathrm{C}$ & 5.803636 & 4.584150 & 1.03 \\
\hline $\mathrm{C}$ & 6.192417 & 3.925413 & -1.32 \\
\hline $\mathrm{C}$ & 6.352539 & 2.270588 & -3.1 \\
\hline $\mathrm{H}$ & -5.989730 & 2.865143 & -4.6 \\
\hline $\mathrm{H}$ & -7.050554 & -4.388625 & 1.2 \\
\hline $\mathrm{H}$ & -6.736748 & -4.791158 & -1.15 \\
\hline $\mathrm{H}$ & -6.415823 & -3.861059 & -3.39 \\
\hline $\mathrm{H}$ & -7.284854 & -2.778296 & 3.08 \\
\hline $\mathrm{H}$ & -7.409919 & -0.467665 & \\
\hline $\mathrm{H}$ & -7.459041 & 1.861752 & 3.35 \\
\hline $\mathrm{H}$ & 6.370609 & -4.294010 & 2.98 \\
\hline $\mathrm{H}$ & 6.685724 & -4.932521 & 0.63 \\
\hline $\mathrm{H}$ & 7.002757 & -4.264730 & -1.69 \\
\hline $\mathrm{H}$ & 7.425090 & 2.195254 & -2.99 \\
\hline $\mathrm{H}$ & 6.112507 & -2.578073 & 4.74 \\
\hline $\mathrm{H}$ & 7.256465 & 3.815833 & -1.13 \\
\hline $\mathrm{H}$ & 5.957544 & -0.227131 & 5.54 \\
\hline $\mathrm{H}$ & 6.863503 & 4.504122 & 1.2565 \\
\hline $\mathrm{H}$ & 6.000724 & 2.188668 & 5.1 \\
\hline $\mathrm{H}$ & 6.352569 & 3.946431 & 3.5921 \\
\hline $\mathrm{C}$ & -6.245465 & 3.795636 & 1.85 \\
\hline $\mathrm{H}$ & -7.309342 & 3.709107 & 1.6563 \\
\hline $\mathrm{C}$ & -5.708590 & 4.741205 & -0.4461 \\
\hline $\mathrm{H}$ & -6.737582 & 4.704048 & -0 \\
\hline C & -5.137076 & 4.429440 & -2.7 \\
\hline $\mathrm{H}$ & -6 & 85 & -2.9 \\
\hline
\end{tabular}




$\begin{array}{rrrr}\mathrm{C} & -4.935879 & 0.429443 & -5.229341 \\ \mathrm{H} & -6.007496 & 0.474513 & -5.397590 \\ \mathrm{C} & -5.081032 & -1.990356 & -4.783717 \\ \mathrm{H} & -6.159704 & -1.946200 & -4.901131 \\ \mathrm{C} & 6.330972 & -0.037619 & -3.946901 \\ \mathrm{H} & 7.408064 & -0.083325 & -3.817313 \\ \mathrm{C} & 6.182199 & -2.436413 & -3.472201 \\ \mathrm{H} & 7.259260 & -2.470786 & -3.338704 \\ \mathrm{O} & -0.683628 & 5.959703 & -0.364445\end{array}$

Figure 7c

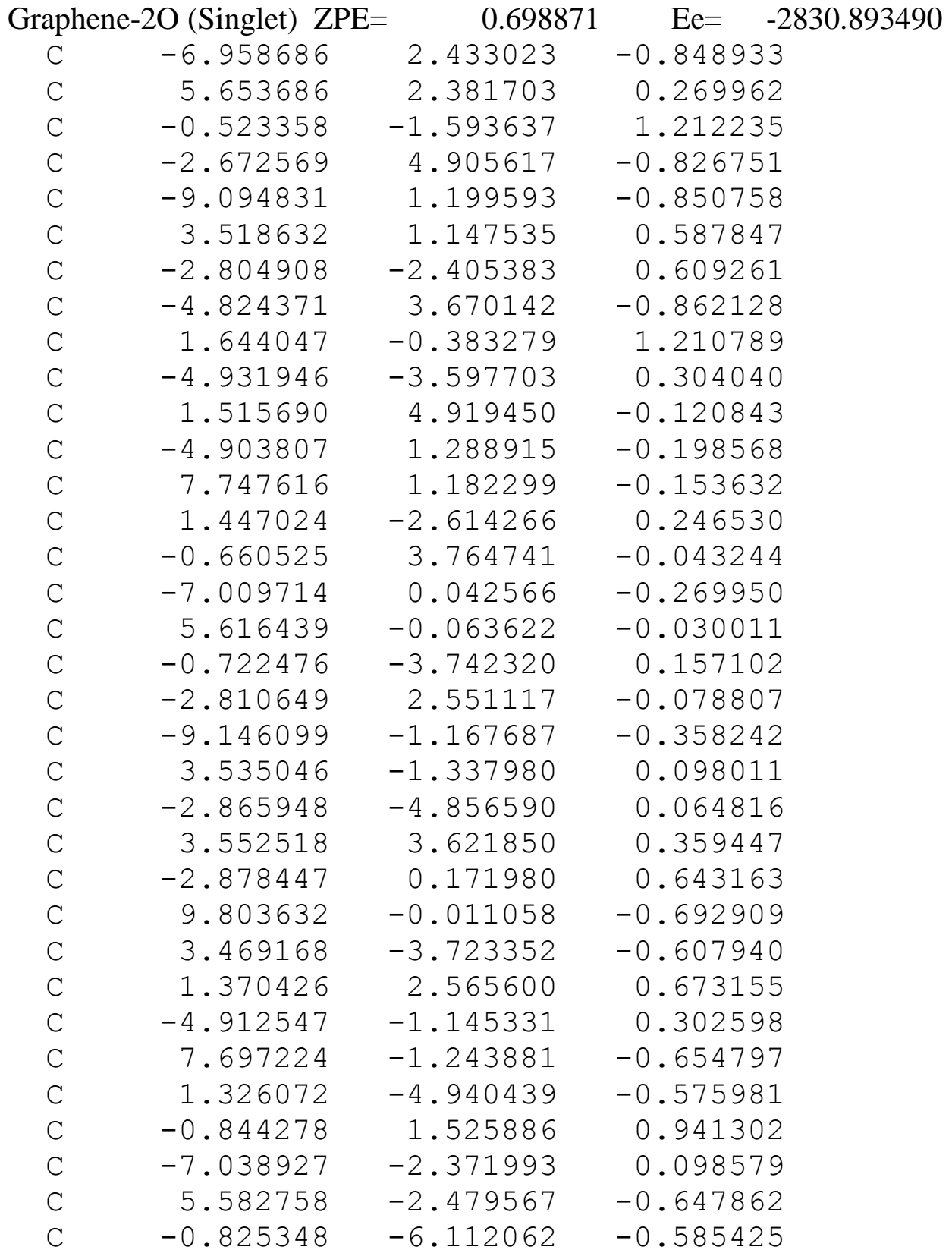




\begin{tabular}{|c|c|c|c|}
\hline C & -1.326073 & 4.940440 & -0.57598 \\
\hline C & -7.697222 & 1.243878 & -0.65481 \\
\hline C & 4.912546 & 1.145331 & 0.30260 \\
\hline $\mathrm{C}$ & -1.370427 & -2.565598 & 0.67315 \\
\hline $\mathrm{C}$ & -3.469167 & 3.723350 & -0.6079 \\
\hline $\mathrm{C}$ & -9.803630 & 0.011055 & -0.69292 \\
\hline C & 2.878446 & -0.171979 & 0.64316 \\
\hline C & -3.552519 & -3.621849 & 0.3594 \\
\hline $\mathrm{C}$ & -5.582757 & 2.479565 & -0.6478 \\
\hline C & 0.844278 & -1.525884 & 0.9413 \\
\hline C & 0.722474 & 3.742322 & 0.157 \\
\hline $\mathrm{C}$ & -5.616438 & 0.063621 & -0.03001 \\
\hline $\mathrm{C}$ & 7.009715 & -0.042567 & -0.2699 \\
\hline $\mathrm{C}$ & 0.660525 & -3.764740 & -0.04323 \\
\hline $\mathrm{C}$ & -1.447024 & 2.614267 & 0.2465 \\
\hline $\mathrm{C}$ & -7.747615 & -1.182300 & -0.153 \\
\hline $\mathrm{C}$ & 4.903808 & -1.288916 & -0.1985 \\
\hline $\mathrm{C}$ & -1.515691 & -4.919448 & -0.1208 \\
\hline $\mathrm{C}$ & -3.535046 & 1.337979 & 0.098 \\
\hline $\mathrm{C}$ & 2.810650 & -2.551117 & -0.078 \\
\hline $\mathrm{C}$ & 2.804906 & 2.405384 & 0.609 \\
\hline $\mathrm{C}$ & -3.518634 & -1.147535 & $0.58^{\circ}$ \\
\hline $\mathrm{C}$ & 9.094834 & -1.199596 & -0.8507 \\
\hline $\mathrm{C}$ & 2.672569 & -4.905618 & -0.826 \\
\hline $\mathrm{C}$ & 0.523356 & 1.593639 & 1.21 \\
\hline $\mathrm{C}$ & -5.653686 & -2.381703 & 0.2699 \\
\hline $\mathrm{C}$ & 6.958688 & -2.433026 & -0.8 \\
\hline $\mathrm{C}$ & 0.507492 & -6.119779 & -0.8093 \\
\hline $\mathrm{C}$ & -1.644049 & 0.383281 & 1.2107 \\
\hline C & 4.824373 & -3.670144 & -0.86 \\
\hline $\mathrm{C}$ & 0.825347 & 6.112065 & -0.5854 \\
\hline $\mathrm{C}$ & 7.038927 & 2.371993 & 0.098 \\
\hline $\mathrm{C}$ & 4.931946 & 3.597704 & 0.3040 \\
\hline $\mathrm{C}$ & 2.865948 & 4.856591 & 0.064 \\
\hline $\mathrm{C}$ & 9.146100 & 1.167685 & -0.358 \\
\hline $\mathrm{H}$ & 7.577694 & 3.314867 & 0.1120 \\
\hline $\mathrm{H}$ & 5.477093 & 4.528932 & $0.1 \varepsilon$ \\
\hline $\mathrm{H}$ & 9.698589 & 2.096027 & -0.2603 \\
\hline C & -0.507493 & 6.119780 & -0.8093 \\
\hline $\mathrm{H}$ & -9.611254 & 2.110722 & -1.1333 \\
\hline $\mathrm{H}$ & -7.483218 & 3.330386 & -1.1638 \\
\hline $\mathrm{H}$ & -5.339895 & 4.551760 & -1.2314 \\
\hline $\mathrm{H}$ & -3.169545 & 5.789767 & -1.2158 \\
\hline $\mathrm{H}$ & -1.001457 & 7.014393 & -1.1740 \\
\hline $\mathrm{H}$ & 1.421511 & 7.000605 & -0.7657 \\
\hline $\mathrm{H}$ & 3.477061 & 5.738670 & -0.1038 \\
\hline O & -1.128000 & -0.629766 & 2.028 \\
\hline
\end{tabular}




$\begin{array}{rrrr}\mathrm{O} & 1.128000 & 0.629770 & 2.028033 \\ \mathrm{H} & 10.876489 & -0.002691 & -0.845793 \\ \mathrm{H} & 9.611258 & -2.110725 & -1.133321 \\ \mathrm{H} & 7.483220 & -3.330390 & -1.163835 \\ \mathrm{H} & 5.339896 & -4.551763 & -1.231441 \\ \mathrm{H} & 3.169545 & -5.789768 & -1.215802 \\ \mathrm{H} & 1.001456 & -7.014391 & -1.174077 \\ \mathrm{H} & -1.421512 & -7.000603 & -0.765739 \\ \mathrm{H} & -3.477061 & -5.738669 & -0.103803 \\ \mathrm{H} & -5.477093 & -4.528932 & 0.181428 \\ \mathrm{H} & -7.577695 & -3.314868 & 0.112091 \\ \mathrm{H} & -9.698589 & -2.096028 & -0.260402 \\ \mathrm{H} & -10.876487 & 0.002688 & -0.845815\end{array}$

\section{Graphene-2O (Triplet) ZPE $=0.697354 \quad \mathrm{Ee}=\quad-2830.918999$}

\begin{tabular}{|c|c|c|c|}
\hline C & 6.979049 & -2.422834 & -0.849574 \\
\hline C & -5.654158 & -2.378133 & 0.281107 \\
\hline $\mathrm{C}$ & 0.521788 & 1.596238 & 1.220994 \\
\hline C & 2.677575 & -4.888842 & -0.840776 \\
\hline $\mathrm{C}$ & 9.117527 & -1.196629 & -0.852771 \\
\hline C & -3.524469 & -1.147378 & 0.597776 \\
\hline C & 2.815227 & 2.400588 & 0.616877 \\
\hline $\mathrm{C}$ & 4.837114 & -3.656732 & -0.862600 \\
\hline $\mathrm{C}$ & -1.651727 & 0.386923 & 1.224909 \\
\hline $\mathrm{C}$ & 4.943297 & 3.585303 & 0.312616 \\
\hline C & -1.518207 & -4.897704 & -0.146916 \\
\hline $\mathrm{C}$ & 4.916277 & -1.290299 & -0.190398 \\
\hline $\mathrm{C}$ & -7.758723 & -1.185669 & -0.144786 \\
\hline $\mathrm{C}$ & -1.447374 & 2.612748 & 0.256492 \\
\hline $\mathrm{C}$ & 0.666829 & -3.751986 & -0.052023 \\
\hline C & 7.018876 & -0.043562 & -0.259999 \\
\hline $\mathrm{C}$ & -5.630260 & 0.066126 & -0.016802 \\
\hline $\mathrm{C}$ & 0.726222 & 3.726070 & 0.144125 \\
\hline $\mathrm{C}$ & 2.823173 & -2.547325 & -0.072505 \\
\hline $\mathrm{C}$ & 9.165728 & 1.168799 & -0.352577 \\
\hline $\mathrm{C}$ & -3.539918 & 1.339555 & 0.107872 \\
\hline $\mathrm{C}$ & 2.869599 & 4.838715 & 0.052254 \\
\hline $\mathrm{C}$ & -3.551706 & -3.610488 & 0.367391 \\
\hline $\mathrm{C}$ & 2.884033 & -0.176055 & 0.658444 \\
\hline $\mathrm{C}$ & -9.823280 & 0.010972 & -0.692631 \\
\hline $\mathrm{C}$ & -3.473296 & 3.712838 & -0.607219 \\
\hline $\mathrm{C}$ & -1.363162 & -2.560508 & 0.673590 \\
\hline $\mathrm{C}$ & 4.922047 & 1.145966 & 0.317683 \\
\hline $\mathrm{C}$ & -7.705928 & 1.244841 & -0.652960 \\
\hline $\mathrm{C}$ & -1.327014 & 4.921801 & -0.596760 \\
\hline $\mathrm{C}$ & 0.851241 & -1.531574 & 0.956040 \\
\hline
\end{tabular}




\begin{tabular}{|c|c|c|c|}
\hline $\mathrm{C}$ & 7.059723 & 2.366083 & 0.10861 \\
\hline C & -5.585624 & 2.473439 & -0.64315 \\
\hline $\mathrm{C}$ & 0.830060 & 6.083867 & -0.63004 \\
\hline $\mathrm{C}$ & 1.327001 & -4.921795 & -0.5967 \\
\hline C & 7.705945 & -1.244873 & -0.6529 \\
\hline C & -4.922028 & -1.145942 & 0.3177 \\
\hline $\mathrm{C}$ & 1.363190 & 2.560420 & 0.6736 \\
\hline $\mathrm{C}$ & 3.473268 & -3.712867 & -0.6071 \\
\hline $\mathrm{C}$ & 9.823262 & -0.010892 & -0.692 \\
\hline C & -2.884070 & 0.176109 & $0.65 \varepsilon$ \\
\hline C & 3.551763 & 3.610439 & 0.367 \\
\hline C & 5.585578 & -2.473466 & -0.643 \\
\hline $\mathrm{C}$ & -0.851302 & 1.531544 & 0.95 \\
\hline $\mathrm{C}$ & -0.726267 & -3.726135 & 0.14 \\
\hline $\mathrm{C}$ & 5.630271 & -0.066111 & -0.01 \\
\hline C & -7.018898 & 0.043592 & -0.25 \\
\hline $\mathrm{C}$ & -0.666748 & 3.751942 & -0.05 \\
\hline C & 1.447357 & -2.612802 & 0.2 \\
\hline $\mathrm{C}$ & 7.758672 & 1.185705 & -0.14 \\
\hline C & -4.916330 & 1.290283 & -0.1 \\
\hline C & 1.518243 & 4.897701 & -0.1 \\
\hline $\mathrm{C}$ & 3.539902 & -1.339542 & 0.1 \\
\hline C & -2.823157 & 2.547301 & -0.07 \\
\hline $\mathrm{C}$ & -2.815217 & -2.400647 & 0.6 \\
\hline C & 3.524459 & 1.147382 & 0.5 \\
\hline C & -9.117534 & 1.196642 & -0.85 \\
\hline $\mathrm{C}$ & -2.677522 & 4.888849 & -0.84 \\
\hline C & -0.521723 & -1.596211 & 1.22 \\
\hline C & 5.654163 & 2.378095 & 0.28 \\
\hline C & -6.979018 & 2.422824 & -0.8 \\
\hline $\mathrm{C}$ & -0.503899 & 6.093151 & -0.85 \\
\hline $\mathrm{C}$ & 1.651756 & -0.386919 & 1.22 \\
\hline $\mathrm{C}$ & -4.837046 & 3.656729 & -0.86 \\
\hline C & -0.830078 & -6.083827 & -0.63 \\
\hline C & -7.059767 & -2.366065 & 0.1 \\
\hline C & -4.943332 & -3.585280 & 0.31 \\
\hline C & -2.869632 & -4.838681 & 0.0 \\
\hline $\mathrm{C}$ & -9.165728 & -1.168740 & -0.35 \\
\hline $\mathrm{H}$ & -7.595407 & -3.310424 & 0.1321 \\
\hline $\mathrm{H}$ & -5.485834 & -4.518303 & 0 \\
\hline $\mathrm{H}$ & -9.717592 & -2.097032 & -0.2519 \\
\hline $\mathrm{C}$ & 0.503909 & -6.093103 & -0.8 \\
\hline $\mathrm{H}$ & 9.631251 & -2.108406 & -1.1 \\
\hline $\mathrm{H}$ & 7.499355 & -3.321360 & -1.1679 \\
\hline $\mathrm{H}$ & 5.350911 & -4.537165 & $-1 \cdot 2$ \\
\hline $\mathrm{H}$ & 3.172422 & -5.770116 & $-1 \cdot 2$ \\
\hline $\mathrm{H}$ & 0.99 & -6.98 & -1 \\
\hline
\end{tabular}




$\begin{array}{rrrr}\mathrm{H} & -1.428068 & -6.967993 & -0.825351 \\ \mathrm{H} & -3.480378 & -5.719721 & -0.122891 \\ \mathrm{O} & 1.126785 & 0.629226 & 2.030841 \\ \mathrm{O} & -1.126766 & -0.629229 & 2.030525 \\ \mathrm{H} & -10.896043 & 0.000612 & -0.846638 \\ \mathrm{H} & -9.631210 & 2.108424 & -1.137797 \\ \mathrm{H} & -7.499373 & 3.321297 & -1.168149 \\ \mathrm{H} & -5.350843 & 4.537128 & -1.237286 \\ \mathrm{H} & -3.172372 & 5.770125 & -1.238984 \\ \mathrm{H} & -0.994637 & 6.984313 & -1.228236 \\ \mathrm{H} & 1.428074 & 6.968053 & -0.825024 \\ \mathrm{H} & 3.480423 & 5.719710 & -0.122749 \\ \mathrm{H} & 5.485824 & 4.518297 & 0.191849 \\ \mathrm{H} & 7.595300 & 3.310482 & 0.131844 \\ \mathrm{H} & 9.717513 & 2.097144 & -0.252202 \\ \mathrm{H} & 10.896023 & -0.000577 & -0.846760\end{array}$

Graphene-2O (Quintet) ZPE=0.695079 $\quad \mathrm{Ee}=\quad-2830.881658$

\begin{tabular}{|c|c|c|c|}
\hline $\mathrm{C}$ & 7.016048 & -2.460084 & -0.743098 \\
\hline $\mathrm{C}$ & -5.718492 & -2.394756 & 0.192806 \\
\hline $\mathrm{C}$ & 0.551167 & 1.554131 & 1.102213 \\
\hline $\mathrm{C}$ & 2.697763 & -4.866831 & -0.707842 \\
\hline C & 9.158853 & -1.231692 & -0.769863 \\
\hline $\mathrm{C}$ & -3.569004 & -1.159254 & 0.427845 \\
\hline $\mathrm{C}$ & 2.846575 & 2.417380 & 0.606396 \\
\hline $\mathrm{C}$ & 4.866395 & -3.674293 & -0.732235 \\
\hline C & -1.468834 & 0.097000 & 0.908668 \\
\hline $\mathrm{C}$ & 4.988419 & 3.586508 & 0.287336 \\
\hline $\mathrm{C}$ & -1.520460 & -4.869027 & -0.106835 \\
\hline $\mathrm{C}$ & 4.953173 & -1.287167 & -0.156333 \\
\hline C & -7.817300 & -1.145854 & -0.136744 \\
\hline C & -1.391003 & 2.514724 & 0.134685 \\
\hline $\mathrm{C}$ & 0.648110 & -3.697613 & -0.079285 \\
\hline $\mathrm{C}$ & 7.068581 & -0.058937 & -0.234539 \\
\hline $\mathrm{C}$ & -5.657174 & 0.061116 & -0.045163 \\
\hline $\mathrm{C}$ & 0.766632 & 3.717589 & 0.128284 \\
\hline C & 2.803652 & -2.511071 & -0.073125 \\
\hline $\mathrm{C}$ & 9.207016 & 1.145992 & -0.335547 \\
\hline $\mathrm{C}$ & -3.523490 & 1.266549 & 0.070811 \\
\hline $\mathrm{C}$ & 2.901098 & 4.837766 & 0.041811 \\
\hline C & -3.597464 & -3.642137 & 0.255700 \\
\hline $\mathrm{C}$ & 2.927828 & -0.158789 & 0.620629 \\
\hline $\mathrm{C}$ & -9.880122 & 0.094949 & -0.553386 \\
\hline $\mathrm{C}$ & -3.441990 & 3.677352 & -0.528410 \\
\hline $\mathrm{C}$ & -1.453890 & -2.482040 & 0.475 \\
\hline $\mathrm{C}$ & 4.956829 & 1.142980 & 0.30 \\
\hline
\end{tabular}




\begin{tabular}{|c|c|c|c|}
\hline $\mathrm{C}$ & -7.751666 & 1.295143 & -0.52417 \\
\hline $\mathrm{C}$ & -1.309816 & 4.884810 & -0.53996 \\
\hline $\mathrm{C}$ & 0.783495 & -1.376424 & 0.73471 \\
\hline $\mathrm{C}$ & 7.098850 & 2.360845 & 0.091 \\
\hline C & -5.595701 & 2.492504 & -0.5191 \\
\hline C & 0.830251 & 6.083428 & -0.5818 \\
\hline $\mathrm{C}$ & 1.304667 & -4.879652 & -0.49338 \\
\hline $\mathrm{C}$ & 7.751015 & -1.267928 & -0.58030 \\
\hline $\mathrm{C}$ & -4.974375 & -1.155770 & 0.20235 \\
\hline C & 1.410858 & 2.566534 & 0.627 \\
\hline C & 3.463715 & -3.708038 & -0.5180 \\
\hline C & 9.866634 & -0.042209 & $-0.6387 \mathrm{~s}$ \\
\hline $\mathrm{C}$ & -2.870490 & 0.064677 & 0.45 \\
\hline $\mathrm{C}$ & 3.579642 & 3.618851 & 0.32 \\
\hline $\mathrm{C}$ & 5.623064 & -2.497620 & -0.55 \\
\hline C & -0.752212 & 1.380242 & 0.724 \\
\hline $\mathrm{C}$ & -0.778757 & -3.673858 & 0.1 \\
\hline C & 5.659842 & -0.073578 & -0.00 \\
\hline $\mathrm{C}$ & -7.076047 & 0.070665 & -0.23 \\
\hline C & -0.643656 & 3.699278 & -0.0 \\
\hline C & 1.407587 & -2.520344 & 0.15 \\
\hline $\mathrm{C}$ & 7.802426 & 1.166301 & -0.1 \\
\hline C & -4.918681 & 1.267190 & -0.1 \\
\hline $\mathrm{C}$ & 1.535725 & 4.896359 & -0.14 \\
\hline C & 3.562809 & -1.316919 & 0.11 \\
\hline $\mathrm{C}$ & -2.785460 & 2.489382 & -0.11 \\
\hline $\mathrm{C}$ & -2.857204 & -2.427699 & 0.4 \\
\hline C & 3.565488 & 1.143549 & 0.59 \\
\hline C & -9.162557 & 1.280925 & -0.67 \\
\hline C & -2.676646 & 4.856674 & -0.74 \\
\hline $\mathrm{C}$ & -0.685764 & -1.302265 & 0.91 \\
\hline C & 5.693216 & 2.382501 & 0.25 \\
\hline C & -6.998696 & 2.477021 & -0.67 \\
\hline C & -0.511058 & 6.075570 & $-0.76 \varepsilon$ \\
\hline C & 1.622268 & -0.354591 & 1.1 \\
\hline $\mathrm{C}$ & -4.845716 & 3.661034 & -0.7073 \\
\hline $\mathrm{C}$ & -0.820755 & -6.070776 & -0.483 \\
\hline $\mathrm{C}$ & -7.121874 & -2.355238 & 0.06 \\
\hline C & -5.018711 & -3.604448 & 0.233 \\
\hline C & -2.910556 & -4.840845 & 0.0 \\
\hline C & -9.226887 & -1.106066 & -0.2933 \\
\hline $\mathrm{H}$ & -7.677577 & -3.287899 & 0.090 \\
\hline $\mathrm{H}$ & -5.565753 & -4.539968 & 0.1689 \\
\hline $\mathrm{H}$ & -9.787775 & -2.031097 & -0.214 \\
\hline $\mathrm{C}$ & 0.523190 & -6.075766 & -0.67 \\
\hline $\mathrm{H}$ & 9.673509 & -2.152150 & -1.0 \\
\hline $\mathrm{H}$ & 7.53 & -3.369522 & -1 \\
\hline
\end{tabular}




$\begin{array}{rrrr}\mathrm{H} & 5.371781 & -4.584013 & -1.042209 \\ \mathrm{H} & 3.189149 & -5.780034 & -1.030338 \\ \mathrm{H} & 1.036332 & -6.983284 & -0.977431 \\ \mathrm{H} & -1.403992 & -6.974253 & -0.627001 \\ \mathrm{H} & -3.478475 & -5.756174 & -0.109852 \\ \mathrm{O} & 1.141939 & 0.631509 & 1.962439 \\ \mathrm{O} & -1.221101 & -0.676416 & 2.135656 \\ \mathrm{H} & -10.957494 & 0.105283 & -0.670192 \\ \mathrm{H} & -9.673721 & 2.212554 & -0.893390 \\ \mathrm{H} & -7.510121 & 3.402022 & -0.923836 \\ \mathrm{H} & -5.352654 & 4.576515 & -0.996712 \\ \mathrm{H} & -3.188874 & 5.754545 & -1.075252 \\ \mathrm{H} & -1.025750 & 6.970641 & -1.101601 \\ \mathrm{H} & 1.409220 & 6.982499 & -0.764657 \\ \mathrm{H} & 3.503943 & 5.725040 & -0.128655 \\ \mathrm{H} & 5.530542 & 4.520037 & 0.170064 \\ \mathrm{H} & 7.639613 & 3.302355 & 0.102612 \\ \mathrm{H} & 9.759073 & 2.076058 & -0.254313 \\ \mathrm{H} & 10.940421 & -0.037576 & -0.784926\end{array}$

$\begin{array}{crrrr}\text { SW Defected Graphene-2O } & \text { (Singlet) ZPE }= & 0.698813 & \text { Ee }= & -2830.805741 \\ \text { C } & -4.904551 & 3.667814 & 0.383377 \\ \text { C } & 4.836398 & -3.716942 & 0.455396 \\ \text { C } & 6.746507 & -2.419183 & 1.385419 \\ \text { C } & 0.720883 & -6.110513 & -0.690103 \\ \text { C } & 2.800402 & -4.917303 & -0.322907 \\ \text { C } & 8.593713 & -1.090821 & 2.363080 \\ \text { C } & 3.608207 & -3.739723 & -0.168410 \\ \text { C } & 9.175524 & 0.140812 & 2.657576 \\ \text { C } & 5.549961 & -2.490499 & 0.701027 \\ \text { C } & -0.621527 & -6.099133 & -0.686914 \\ \text { C } & 7.377788 & -1.166526 & 1.656392 \\ \text { C } & 1.476476 & -4.871302 & -0.672033 \\ \text { C } & 3.035958 & -2.530049 & -0.704546 \\ \text { C } & 8.556874 & 1.326499 & 2.272852 \\ \text { C } & 4.966927 & -1.263946 & 0.213920 \\ \text { C } & -1.354827 & -4.847666 & -0.646903 \\ \text { C } & 0.775986 & -3.603163 & -0.952658 \\ \text { C } & 6.754628 & 0.047611 & 1.231549 \\ \text { C } & 1.713998 & -2.484069 & -1.158349 \\ \text { C } & 7.338600 & 1.301408 & 1.565042 \\ \text { C } & 3.816917 & -1.306545 & -0.571250 \\ \text { C } & -2.684205 & -4.883783 & -0.312725 \\ \text { C } & -0.637445 & -3.588063 & -0.902712 \\ \text { C } & 5.538372 & 0.004328 & 0.485219 \\ \text { C } & 1.373772 & -1.228693 & -1.768668 \\ \text { C } & 6.637448 & 2.485755 & 1.208067 \\ & & & \end{array}$




\begin{tabular}{|c|c|c|c|}
\hline $\mathrm{C}$ & 3.325363 & -0.120224 & -1.12761 \\
\hline $\mathrm{C}$ & -3.481462 & -3.704866 & -0.12469 \\
\hline $\mathrm{C}$ & -1.509832 & -2.400995 & -0.94590 \\
\hline $\mathrm{C}$ & 4.864670 & 1.202845 & 0.09158 \\
\hline C & -1.072892 & -1.133229 & -1.35682 \\
\hline $\mathrm{C}$ & 5.430123 & 2.455190 & $0.5354 \varepsilon$ \\
\hline $\mathrm{C}$ & 0.178273 & -0.622258 & -1.866 \\
\hline $\mathrm{C}$ & -2.854795 & -2.452070 & -0.4612 \\
\hline $\mathrm{C}$ & 3.669740 & 1.176888 & -0.7116 \\
\hline $\mathrm{C}$ & -1.839440 & 0.004120 & -1.13 \\
\hline $\mathrm{C}$ & 4.680707 & 3.649398 & $0.311 \varepsilon$ \\
\hline $\mathrm{C}$ & 0.072214 & 0.824861 & -1.93 \\
\hline $\mathrm{C}$ & -5.549191 & -2.547657 & 0.594 \\
\hline $\mathrm{C}$ & -3.635826 & -1.269434 & -0.265 \\
\hline $\mathrm{C}$ & 2.838907 & 2.427982 & -0.81 \\
\hline $\mathrm{C}$ & -3.087622 & -0.009983 & -0.5699 \\
\hline $\mathrm{C}$ & 3.425032 & 3.641164 & -0.23 \\
\hline C & -1.122316 & 1.211008 & -1.41 \\
\hline C & -4.937447 & -1.287852 & 0.28 \\
\hline C & 1.542219 & 2.569336 & -1.29 \\
\hline $\mathrm{C}$ & -3.699606 & 1.234403 & -0.2 \\
\hline $\mathrm{C}$ & 2.668277 & 4.858569 & -0.27 \\
\hline $\mathrm{C}$ & -1.632590 & 2.475816 & -0.98 \\
\hline $\mathrm{C}$ & -7.535304 & -1.336724 & 1.37 \\
\hline $\mathrm{C}$ & -5.606421 & -0.058074 & 0.5 \\
\hline $\mathrm{C}$ & 0.638098 & 3.676181 & -0.959 \\
\hline $\mathrm{C}$ & -4.975137 & 1.206719 & 0.26 \\
\hline $\mathrm{C}$ & 1.336008 & 4.895594 & -0.56 \\
\hline $\mathrm{C}$ & -2.953180 & 2.465517 & -0.49 \\
\hline $\mathrm{C}$ & -6.907435 & -0.080983 & 1.09 \\
\hline $\mathrm{C}$ & -0.781326 & 3.655838 & -0.895 \\
\hline $\mathrm{C}$ & -5.638221 & 2.446680 & 0.59 \\
\hline $\mathrm{C}$ & 0.591742 & 6.135384 & -0.45 \\
\hline $\mathrm{C}$ & -9.491567 & -0.128666 & 2.18 \\
\hline $\mathrm{C}$ & -3.610720 & 3.691559 & -0.10 \\
\hline $\mathrm{C}$ & -7.581036 & 1.153174 & 1.37 \\
\hline $\mathrm{C}$ & -1.495399 & 4.888286 & -0.53 \\
\hline $\mathrm{C}$ & -6.914962 & 2.395356 & 1.12 \\
\hline $\mathrm{C}$ & -8.873407 & 1.099448 & 1.921 \\
\hline $\mathrm{C}$ & -4.777760 & -3.737994 & 0.3 \\
\hline C & -6.837014 & -2.546860 & 1.1231 \\
\hline C & -8.841985 & -1.325407 & 1.9 \\
\hline $\mathrm{C}$ & -0.752394 & 6.133430 & -0.4561 \\
\hline $\mathrm{C}$ & -2.830054 & 4.891328 & -0.21 \\
\hline $\mathrm{H}$ & -1.192722 & -7.020584 & -0.6 \\
\hline $\mathrm{H}$ & -3.146842 & -5.849660 & -0.1 \\
\hline $\mathrm{H}$ & -9.39 & 01 & 2.1 \\
\hline
\end{tabular}




$\begin{array}{rrrr}\mathrm{H} & -5.231247 & -4.697767 & 0.590226 \\ \mathrm{H} & -7.323407 & -3.488427 & 1.361508 \\ \mathrm{H} & -9.327338 & -2.271181 & 2.142090 \\ \mathrm{H} & -1.316582 & 7.051496 & -0.330695 \\ \mathrm{H} & -3.303325 & 5.836232 & 0.036163 \\ \mathrm{H} & -7.433591 & 3.316631 & 1.374325 \\ \mathrm{H} & -5.384379 & 4.604166 & 0.655050 \\ \mathrm{H} & -10.490885 & -0.141379 & 2.605385 \\ \mathrm{O} & 2.411553 & -0.383749 & -2.137328 \\ \mathrm{O} & 1.101685 & 1.671783 & -2.273655 \\ \mathrm{H} & 3.169638 & 5.771121 & 0.034171 \\ \mathrm{H} & 5.099621 & 4.589540 & 0.657815 \\ \mathrm{H} & 7.049791 & 3.445187 & 1.506860 \\ \mathrm{H} & 9.002607 & 2.283830 & 2.521158 \\ \mathrm{H} & 10.112456 & 0.174963 & 3.200953 \\ \mathrm{H} & 9.072347 & -2.010376 & 2.682942 \\ \mathrm{H} & 7.221335 & -3.327803 & 1.743934 \\ \mathrm{H} & 5.265251 & -4.643431 & 0.825436 \\ \mathrm{H} & 3.243164 & -5.879912 & -0.083815 \\ \mathrm{H} & 1.278045 & -7.040377 & -0.652881 \\ \mathrm{H} & 1.155997 & 7.052440 & -0.320429\end{array}$

\section{SW Defected Graphene-2O (Triplet) ZPE $=0.694442 \quad \mathrm{Ee}=\quad-2830.812640$}

\begin{tabular}{|c|c|c|c|}
\hline $\mathrm{C}$ & 5.027990 & -3.649771 & 0.317190 \\
\hline $\mathrm{C}$ & -4.944307 & 3.691923 & 0.415562 \\
\hline C & -6.906995 & 2.401151 & 1.226641 \\
\hline $\mathrm{C}$ & -0.736983 & 6.059859 & -0.412925 \\
\hline $\mathrm{C}$ & -2.841152 & 4.874279 & -0.198617 \\
\hline C & -8.811636 & 1.077941 & 2.081909 \\
\hline C & -3.653782 & 3.717972 & -0.146934 \\
\hline C & -9.413304 & -0.152147 & 2.332980 \\
\hline $\mathrm{C}$ & -5.654331 & 2.479381 & 0.609096 \\
\hline $\mathrm{C}$ & 0.609690 & 6.055463 & -0.429544 \\
\hline C & -7.544918 & 1.154912 & 1.450864 \\
\hline C & -1.485596 & 4.829385 & -0.498840 \\
\hline $\mathrm{C}$ & -3.073500 & 2.523616 & -0.665369 \\
\hline C & -8.775150 & -1.337931 & 1.983022 \\
\hline $\mathrm{C}$ & -5.046569 & 1.253474 & 0.159031 \\
\hline C & 1.345646 & 4.815931 & -0.497786 \\
\hline C & -0.800703 & 3.584607 & -0.825374 \\
\hline $\mathrm{C}$ & -6.896814 & -0.059170 & 1.0 \\
\hline C & -1.717533 & 2.482347 & -1.074602 \\
\hline C & -7.506272 & -1.316200 & 1.352443 \\
\hline C & -3.850517 & 1.298987 & -0.5 \\
\hline C & 2.709012 & 4.861129 & -0.2 \\
\hline C & 0.65 & 3.5 & -0 \\
\hline
\end{tabular}




\begin{tabular}{|c|c|c|c|}
\hline C & -5.634029 & -0.014483 & 0.39220 \\
\hline C & -1.364247 & 1.226640 & -1.6736 \\
\hline C & -6.798834 & -2.494107 & 1.04031 \\
\hline $\mathrm{C}$ & -3.335966 & 0.115167 & -1.09891 \\
\hline $\mathrm{C}$ & 3.512171 & 3.701220 & -0.0841 \\
\hline C & 1.518343 & 2.398411 & -0.8393 \\
\hline C & -4.937982 & -1.212868 & 0.0333 \\
\hline C & 1.078056 & 1.124949 & -1.2329 \\
\hline $\mathrm{C}$ & -5.523909 & -2.467447 & 0.44 \\
\hline $\mathrm{C}$ & -0.172184 & 0.616250 & -1.7431 \\
\hline $\mathrm{C}$ & 2.884950 & 2.449106 & -0.399 \\
\hline $\mathrm{C}$ & -3.696581 & -1.186731 & -0.699 \\
\hline $\mathrm{C}$ & 1.850173 & -0.005989 & -1.0259 \\
\hline $\mathrm{C}$ & -4.775613 & -3.635510 & 0.2830 \\
\hline $\mathrm{C}$ & -0.062319 & -0.827869 & -1.8305 \\
\hline $\mathrm{C}$ & 5.629488 & 2.548249 & 0.53 \\
\hline $\mathrm{C}$ & 3.677220 & 1.265805 & -0.2362 \\
\hline $\mathrm{C}$ & -2.866772 & -2.432060 & -0.77 \\
\hline $\mathrm{C}$ & 3.122304 & 0.010630 & -0.51 \\
\hline C & -3.445657 & -3.620113 & -0.19 \\
\hline $\mathrm{C}$ & 1.135147 & -1.210527 & $-1 \cdot 31$ \\
\hline $\mathrm{C}$ & 5.009416 & 1.285359 & 0.24 \\
\hline $\mathrm{C}$ & -1.523718 & -2.577318 & -1.209 \\
\hline $\mathrm{C}$ & 3.756660 & -1.236751 & -0.286 \\
\hline $\mathrm{C}$ & -2.681088 & -4.794200 & $-0.0 s$ \\
\hline $\mathrm{C}$ & 1.652511 & -2.482404 & -0.912 \\
\hline $\mathrm{C}$ & 7.659590 & 1.340962 & 1.2 \\
\hline $\mathrm{C}$ & 5.695330 & 0.060618 & 0.45 \\
\hline C & -0.654520 & -3.649265 & -0.8332 \\
\hline C & 5.058172 & -1.207441 & 0.2 \\
\hline $\mathrm{C}$ & -1.315324 & -4.819324 & -0.3361 \\
\hline $\mathrm{C}$ & 3.018411 & -2.468408 & -0.47 \\
\hline $\mathrm{C}$ & 7.027476 & 0.088396 & 0.9 \\
\hline $\mathrm{C}$ & 0.833833 & -3.640387 & -0.80 \\
\hline $\mathrm{C}$ & 5.736252 & -2.451112 & 0.4 \\
\hline $\mathrm{C}$ & -0.573446 & -6.038121 & -0.1025 \\
\hline $\mathrm{C}$ & 9.669990 & 0.147318 & 1.9082 \\
\hline $\mathrm{C}$ & 3.669233 & -3.681329 & -0.11 \\
\hline C & 7.727002 & -1.147688 & 1.1907 \\
\hline $\mathrm{C}$ & 1.521250 & -4.845867 & -0.412 \\
\hline $\mathrm{C}$ & 7.069536 & -2.385970 & 0.9627 \\
\hline $\mathrm{C}$ & 9.049286 & -1.084690 & 1.667 \\
\hline $\mathrm{C}$ & 4.847556 & 3.734268 & 0.3436 \\
\hline $\mathrm{C}$ & 6.944462 & 2.549267 & 0.9982 \\
\hline $\mathrm{C}$ & 8.997734 & 1.338195 & 1.6904 \\
\hline $\mathrm{C}$ & 0.771641 & -6.059877 & -0.1734 \\
\hline $\mathrm{C}$ & 2.893261 & -4.857598 & -0.151 \\
\hline
\end{tabular}




$\begin{array}{rrrr}\mathrm{H} & 1.174150 & 6.975909 & -0.326652 \\ \mathrm{H} & 3.157496 & 5.829410 & -0.007289 \\ \mathrm{H} & 9.586687 & -2.009104 & 1.850549 \\ \mathrm{H} & 5.304196 & 4.695402 & 0.562339 \\ \mathrm{H} & 7.438395 & 3.491951 & 1.215520 \\ \mathrm{H} & 9.484328 & 2.287752 & 1.886415 \\ \mathrm{H} & 1.327754 & -6.970454 & 0.022245 \\ \mathrm{H} & 3.356362 & -5.800110 & 0.126773 \\ \mathrm{H} & 7.605414 & -3.307213 & 1.171476 \\ \mathrm{H} & 5.515840 & -4.589810 & 0.558982 \\ \mathrm{H} & 10.689867 & 0.166032 & 2.274677 \\ \mathrm{O} & -2.387994 & 0.376550 & -2.071657 \\ \mathrm{O} & -1.070253 & -1.690562 & -2.199320 \\ \mathrm{H} & -3.166076 & -5.695904 & 0.268741 \\ \mathrm{H} & -5.191697 & -4.580198 & 0.619103 \\ \mathrm{H} & -7.229606 & -3.455757 & 1.302731 \\ \mathrm{H} & -9.237886 & -2.295558 & 2.196074 \\ \mathrm{H} & -10.383436 & -0.186056 & 2.814743 \\ \mathrm{H} & -9.306152 & 1.998681 & 2.372417 \\ \mathrm{H} & -7.402062 & 3.310427 & 1.554370 \\ \mathrm{H} & -5.384610 & 4.619805 & 0.767874 \\ \mathrm{H} & -3.273458 & 5.830271 & 0.082544 \\ \mathrm{H} & -1.294239 & 6.982364 & -0.291177 \\ \mathrm{H} & -1.137391 & -6.928941 & 0.152379\end{array}$

SW Defected Graphene-2O (Quintet) ZPE $=0.694509 \quad \mathrm{Ee}=\quad-2830.819329$

$\begin{array}{lrrr}\text { C } & 5.037246 & -3.648791 & 0.318306 \\ C & -4.980434 & 3.689097 & 0.399212 \\ C & -6.951227 & 2.401713 & 1.185492 \\ C & -0.734733 & 6.039046 & -0.326837 \\ C & -2.849932 & 4.859763 & -0.158440 \\ C & -8.860217 & 1.075660 & 2.017548 \\ C & -3.661759 & 3.714934 & -0.146715 \\ C & -9.460256 & -0.155881 & 2.264812 \\ C & -5.680877 & 2.485418 & 0.581857 \\ C & 0.612130 & 6.037992 & -0.357437 \\ C & -7.584207 & 1.156385 & 1.403354 \\ C & -1.483214 & 4.812267 & -0.446370 \\ C & -3.085526 & 2.527243 & -0.660605 \\ C & -8.812699 & -1.340033 & 1.927658 \\ C & -5.065416 & 1.256645 & 0.141350 \\ C & 1.349719 & 4.803800 & -0.467269 \\ C & -0.812291 & 3.579398 & -0.799260 \\ C & -6.925311 & -0.058540 & 1.026204 \\ C & -1.715527 & 2.486495 & -1.058822 \\ C & -7.534623 & -1.315519 & 1.313582 \\ C & -3.862454 & 1.302462 & -0.564788\end{array}$




\begin{tabular}{|c|c|c|c|}
\hline $\mathrm{C}$ & 2.727739 & 4.853175 & -0.19405 \\
\hline $\mathrm{C}$ & 0.669165 & 3.576207 & -0.76114 \\
\hline $\mathrm{C}$ & -5.653244 & -0.012493 & 0.3730 \\
\hline $\mathrm{C}$ & -1.360482 & 1.227075 & -1.6547 \\
\hline C & -6.820935 & -2.492671 & 1.01472 \\
\hline $\mathrm{C}$ & -3.339610 & 0.116033 & -1.094 \\
\hline $\mathrm{C}$ & 3.522317 & 3.702105 & -0.09 \\
\hline $\mathrm{C}$ & 1.521143 & 2.403919 & -0.8220 \\
\hline $\mathrm{C}$ & -4.951061 & -1.209450 & 0.026 \\
\hline $\mathrm{C}$ & 1.079459 & 1.124262 & -1.208 \\
\hline $\mathrm{C}$ & -5.538883 & -2.465255 & 0.430 \\
\hline $\mathrm{C}$ & -0.169428 & 0.617300 & -1.7 \\
\hline C & 2.899609 & 2.454147 & -0.39 \\
\hline $\mathrm{C}$ & -3.701999 & -1.183638 & -0.69 \\
\hline $\mathrm{C}$ & 1.848599 & -0.003279 & -1.00 \\
\hline $\mathrm{C}$ & -4.787460 & -3.631745 & 0.28 \\
\hline $\mathrm{C}$ & -0.060795 & -0.826245 & -1.81 \\
\hline C & 5.649960 & 2.553171 & 0.5 \\
\hline C & 3.691270 & 1.267332 & -0.2 \\
\hline C & -2.870536 & -2.430113 & -0.7 \\
\hline $\mathrm{C}$ & 3.129277 & 0.010580 & -0.5 \\
\hline $\mathrm{C}$ & -3.449874 & -3.615520 & -0.1 \\
\hline $\mathrm{C}$ & 1.135409 & -1.207185 & -1.2 \\
\hline $\mathrm{C}$ & 5.025816 & 1.287240 & 0.2 \\
\hline C & -1.524770 & -2.574723 & -1.1 \\
\hline $\mathrm{C}$ & 3.760886 & -1.232159 & -0.2 \\
\hline $\mathrm{C}$ & -2.684866 & -4.785524 & -0.06 \\
\hline $\mathrm{C}$ & 1.654884 & -2.478954 & $-0.8 \varepsilon$ \\
\hline $\mathrm{C}$ & 7.694735 & 1.339895 & 1.1 \\
\hline $\mathrm{C}$ & 5.713007 & 0.059766 & 0.4 \\
\hline $\mathrm{C}$ & -0.656232 & -3.644258 & -0.80 \\
\hline $\mathrm{C}$ & 5.073060 & -1.203769 & 0.2 \\
\hline $\mathrm{C}$ & -1.315757 & -4.810264 & -0.3 \\
\hline $\mathrm{C}$ & 3.021356 & -2.464543 & -0.4 \\
\hline $\mathrm{C}$ & 7.052692 & 0.083667 & 0.9 \\
\hline $\mathrm{C}$ & 0.835648 & -3.637223 & -0.77 \\
\hline $\mathrm{C}$ & 5.750914 & -2.447062 & $0.4 \varepsilon$ \\
\hline $\mathrm{C}$ & -0.574529 & -6.026799 & -0.06 \\
\hline $\mathrm{C}$ & 9.710111 & 0.135089 & 1.846 \\
\hline $\mathrm{C}$ & 3.674253 & -3.678941 & -0.1 \\
\hline C & 7.746609 & -1.149227 & 1.1555 \\
\hline $\mathrm{C}$ & 1.520660 & -4.839895 & -0.3 \\
\hline $\mathrm{C}$ & 7.087017 & -2.383368 & 0.94 \\
\hline $\mathrm{C}$ & 9.084318 & -1.089646 & 1.6 \\
\hline $\mathrm{C}$ & 4.884721 & 3.728438 & \\
\hline C & 6.985619 & 2.546713 & 0.9 \\
\hline 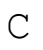 & 9 & 1 & \\
\hline
\end{tabular}




$\begin{array}{lrrr}\mathrm{C} & 0.770877 & -6.050573 & -0.135074 \\ \mathrm{C} & 2.896871 & -4.852655 & -0.130554 \\ \mathrm{H} & 1.174850 & 6.957132 & -0.235248 \\ \mathrm{H} & 3.171440 & 5.821758 & 0.016638 \\ \mathrm{H} & 9.617574 & -2.017227 & 1.799085 \\ \mathrm{H} & 5.342140 & 4.690494 & 0.539399 \\ \mathrm{H} & 7.484458 & 3.489508 & 1.163174 \\ \mathrm{H} & 9.530928 & 2.280405 & 1.816264 \\ \mathrm{H} & 1.326288 & -6.960412 & 0.066112 \\ \mathrm{H} & 3.359317 & -5.795526 & 0.147360 \\ \mathrm{H} & 7.622836 & -3.305509 & 1.145946 \\ \mathrm{H} & 5.526553 & -4.588694 & 0.557293 \\ \mathrm{H} & 10.734224 & 0.153553 & 2.201210 \\ \mathrm{O} & -2.381857 & 0.376514 & -2.055281 \\ \mathrm{O} & -1.068883 & -1.689598 & -2.179537 \\ \mathrm{H} & -3.169482 & -5.686091 & 0.298383 \\ \mathrm{H} & -5.204577 & -4.576368 & 0.619516 \\ \mathrm{H} & -7.253011 & -3.454386 & 1.274725 \\ \mathrm{H} & -9.274280 & -2.298927 & 2.137394 \\ \mathrm{H} & -10.436697 & -0.191987 & 2.733473 \\ \mathrm{H} & -9.362209 & 1.995509 & 2.297825 \\ \mathrm{H} & -7.452885 & 3.310901 & 1.502855 \\ \mathrm{H} & -5.422891 & 4.619544 & 0.741354 \\ \mathrm{H} & -3.276562 & 5.812411 & 0.141835 \\ \mathrm{H} & -1.291707 & 6.957150 & -0.174515 \\ \mathrm{H} & -1.138330 & -6.915083 & 0.203502\end{array}$

Figure 7d

Nanotube-2O (Singlet) $\mathrm{ZPE}=\quad 1.189528 \quad \mathrm{Ee}=\quad-5651.804943$

\begin{tabular}{|c|c|c|c|}
\hline C & -5.812384 & -4.410088 & -2.057854 \\
\hline $\mathrm{C}$ & -5.197724 & -3.630396 & -3.060233 \\
\hline $\mathrm{C}$ & -5.926550 & -2.665020 & -3.783374 \\
\hline $\mathrm{C}$ & -5.296085 & -1.482264 & -4.222827 \\
\hline C & -6.002031 & -0.268074 & -4.345643 \\
\hline $\mathrm{C}$ & -5.346176 & 0.961523 & -4.145770 \\
\hline $\mathrm{C}$ & -6.029167 & 2.084813 & -3.627925 \\
\hline $\mathrm{C}$ & -5.337979 & 3.076248 & -2.911429 \\
\hline C & -5.968390 & 3.836791 & -1.898078 \\
\hline $\mathrm{C}$ & -5.214220 & 4.419297 & -0.862789 \\
\hline $\mathrm{C}$ & -5.734307 & 4.698087 & 0.413293 \\
\hline $\mathrm{C}$ & -4.895088 & 4.660184 & 1.557996 \\
\hline $\mathrm{C}$ & -5.452200 & 4.202409 & 2.759851 \\
\hline C & -4.677560 & 3.393829 & 3.611643 \\
\hline $\mathrm{C}$ & -5.310050 & 2.401905 & 4.389348 \\
\hline $\mathrm{C}$ & -4.642675 & 1.206157 & 4.700203 \\
\hline $\mathrm{C}$ & -5.333690 & -0.008949 & 4.9 \\
\hline
\end{tabular}




\begin{tabular}{|c|c|c|c|}
\hline $\mathrm{C}$ & -4.703019 & -1.243441 & 4.67426 \\
\hline $\mathrm{C}$ & -5.423731 & -2.396727 & 4.29024 \\
\hline $\mathrm{C}$ & -4.803615 & -3.415066 & 3.53981 \\
\hline $\mathrm{C}$ & -5.542702 & -4.215805 & 2.6430 \\
\hline $\mathrm{C}$ & -4.928605 & -4.760311 & 1.4962 \\
\hline $\mathrm{C}$ & -5.677341 & -4.974520 & 0.32021 \\
\hline $\mathrm{C}$ & -5.066773 & -4.851186 & -0.94 \\
\hline $\mathrm{C}$ & -3.753898 & -3.598912 & -3.1122 \\
\hline C & -3.098888 & -2.584845 & -3.858 \\
\hline $\mathrm{C}$ & -3.851967 & -1.444986 & -4.253 \\
\hline $\mathrm{C}$ & -3.173655 & -0.203835 & -4.4021 \\
\hline $\mathrm{C}$ & -3.901459 & 0.995067 & -4.1 \\
\hline C & -3.195645 & 2.158522 & -3.7656 \\
\hline C & -3.897329 & 3.130701 & -3.018 \\
\hline $\mathrm{C}$ & -3.155501 & 3.998269 & -2.1 \\
\hline $\mathrm{C}$ & -3.787884 & 4.521310 & -1.021 \\
\hline $\mathrm{C}$ & -2.984886 & 4.971638 & 0.05 \\
\hline C & -3.452037 & 4.739734 & 1.41 \\
\hline C & -2.612364 & 4.219417 & 2.4 \\
\hline C & -3.244775 & 3.365027 & 3.4 \\
\hline $\mathrm{C}$ & -2.513216 & 2.306271 & 4.02 \\
\hline $\mathrm{C}$ & -3.209736 & 1.173993 & 4.52 \\
\hline $\mathrm{C}$ & -2.521593 & -0.068522 & 4.64 \\
\hline $\mathrm{C}$ & -3.260901 & -1.272569 & 4.5 \\
\hline $\mathrm{C}$ & -2.599591 & -2.451462 & 4.1 \\
\hline $\mathrm{C}$ & -3.360323 & -3.449787 & 3.4 \\
\hline $\mathrm{C}$ & -2.716421 & -4.268185 & 2.45 \\
\hline $\mathrm{C}$ & -3.487054 & -4.782990 & 1.41 \\
\hline $\mathrm{C}$ & -2.850859 & -4.986465 & 0.1 \\
\hline $\mathrm{C}$ & -3.624648 & -4.844758 & -1.0 \\
\hline $\mathrm{C}$ & -2.984932 & -4.360182 & $-2.1 \varepsilon$ \\
\hline $\mathrm{C}$ & -1.670978 & -2.553739 & -3.92 \\
\hline $\mathrm{C}$ & -1.006417 & -1.377029 & -4.37 \\
\hline $\mathrm{C}$ & -1.743832 & -0.170979 & -4.45 \\
\hline $\mathrm{C}$ & -1.051626 & 1.064166 & $-4 \cdot 3$ \\
\hline $\mathrm{C}$ & -1.761504 & 2.194760 & -3.828 \\
\hline $\mathrm{C}$ & -1.035960 & 3.241877 & -3.19 \\
\hline $\mathrm{C}$ & -1.741327 & 4.090028 & -2.30 \\
\hline $\mathrm{C}$ & -1.014826 & 4.917901 & -1.4040 \\
\hline $\mathrm{C}$ & -1.678294 & 5.345681 & -0.2 \\
\hline $\mathrm{C}$ & -0.388115 & 5.055452 & 1.6038 \\
\hline $\mathrm{C}$ & -1.136515 & 4.228056 & 2.4 \\
\hline $\mathrm{C}$ & -0.395863 & 3.271340 & 3.226 \\
\hline $\mathrm{C}$ & -1.080896 & 2.257125 & 3.9186 \\
\hline $\mathrm{C}$ & -0.372330 & 1.097873 & 4.3 \\
\hline $\mathrm{C}$ & -1.094477 & -0.102752 & 4.56 \\
\hline $\mathrm{C}$ & -0.41 & -1.342927 & \\
\hline
\end{tabular}




\begin{tabular}{|c|c|c|c|}
\hline $\mathrm{C}$ & -1.172181 & -2.486864 & 4.04984 \\
\hline $\mathrm{C}$ & -0.518067 & -3.516621 & 3.3227 \\
\hline C & -1.288283 & -4.295914 & $2.4167^{\circ}$ \\
\hline $\mathrm{C}$ & -0.645466 & -4.814784 & 1.25847 \\
\hline C & -1.422561 & -4.990903 & 0.0811 \\
\hline C & -0.782276 & -4.826951 & -1.1795 \\
\hline $\mathrm{C}$ & -1.556896 & -4.341047 & -2.2668 \\
\hline $\mathrm{C}$ & -0.909569 & -3.545929 & -3.2543 \\
\hline $\mathrm{C}$ & 0.419146 & -1.342407 & -4.4303 \\
\hline C & 1.094448 & -0.102150 & -4.5642 \\
\hline C & 0.372509 & 1.098335 & -4.348 \\
\hline C & 1.081172 & 2.257668 & -3.9184 \\
\hline $\mathrm{C}$ & 0.396303 & 3.271712 & -3.2260 \\
\hline $\mathrm{C}$ & 1.137046 & 4.228414 & -2.4253 \\
\hline $\mathrm{C}$ & 0.388806 & 5.055649 & -1.6031 \\
\hline C & 1.679024 & 5.345747 & 0.237 \\
\hline $\mathrm{C}$ & 1.015411 & 4.917697 & 1.40 \\
\hline C & 1.741789 & 4.089536 & 2.3 \\
\hline $\mathrm{C}$ & 1.036297 & 3.241308 & 3.15 \\
\hline C & 1.761734 & 2.193998 & 3.8 \\
\hline $\mathrm{C}$ & 1.051743 & 1.063534 & 4.30 \\
\hline $\mathrm{C}$ & 1.743809 & -0.171796 & 4.45 \\
\hline C & 1.006299 & -1.377637 & 4.37 \\
\hline $\mathrm{C}$ & 1.670712 & -2.554499 & 3.92 \\
\hline C & 0.909173 & -3.546490 & 3.2 \\
\hline $\mathrm{C}$ & 1.556383 & -4.341604 & 2.266 \\
\hline $\mathrm{C}$ & 0.781688 & -4.827251 & 1.17903 \\
\hline C & 1.421963 & -4.991023 & -0.0816 \\
\hline C & 0.644894 & -4.814578 & -1.259 \\
\hline C & 1.287785 & -4.295623 & -2.4 \\
\hline $\mathrm{C}$ & 0.517636 & -3.516123 & -3.323 \\
\hline C & 1.171860 & -2.486321 & -4.04 \\
\hline C & 2.521650 & -0.068014 & -4.64 \\
\hline C & 3.209878 & 1.174196 & -4.5288 \\
\hline C & 2.513501 & 2.306786 & -4.0 \\
\hline $\mathrm{C}$ & 3.245062 & 3.365221 & -3.4194 \\
\hline C & 2.612884 & 4.219760 & -2.4432 \\
\hline C & 3.452393 & 4.739881 & -1.41 \\
\hline C & 2.985252 & 4.971415 & -0.0538 \\
\hline C & 3.788326 & 4.520421 & 1.0 \\
\hline C & 3.155950 & 3.997387 & 2.1715 \\
\hline $\mathrm{C}$ & 3.897829 & 3.129566 & 3.0 \\
\hline C & 3.195934 & 2.157524 & $3.765^{\circ}$ \\
\hline C & 3.901724 & 0.994055 & 4.1906 \\
\hline C & 3.173655 & -0.204954 & 4.4 \\
\hline C & 3.851731 & -1.445944 & 4.2 \\
\hline$C$ & 3.098542 & -2.585922 & 3 \\
\hline
\end{tabular}




\begin{tabular}{|c|c|c|c|}
\hline $\mathrm{C}$ & 3.753279 & -3.599907 & 3.11215 \\
\hline $\mathrm{C}$ & 2.984392 & -4.360980 & 2.18898 \\
\hline $\mathrm{C}$ & 3.623985 & -4.845485 & 1.01875 \\
\hline $\mathrm{C}$ & 2.850289 & -4.986727 & -0.16396 \\
\hline C & 3.486512 & -4.782958 & -1.41702 \\
\hline $\mathrm{C}$ & 2.715948 & -4.268006 & -2.4904 \\
\hline $\mathrm{C}$ & 3.360068 & -3.449395 & -3.458 \\
\hline $\mathrm{C}$ & 2.599400 & -2.451045 & -4.11372 \\
\hline $\mathrm{C}$ & 3.260920 & -1.272269 & -4.56452 \\
\hline $\mathrm{C}$ & 4.642781 & 1.206049 & -4.70001 \\
\hline $\mathrm{C}$ & 5.310427 & 2.401598 & -4.3886 \\
\hline $\mathrm{C}$ & 4.677888 & 3.393689 & -3.61 \\
\hline C & 4.895511 & 4.659976 & -1.5574 \\
\hline $\mathrm{C}$ & 5.214718 & 4.418552 & 0.863 \\
\hline $\mathrm{C}$ & 5.968905 & 3.836168 & 1.898 \\
\hline $\mathrm{C}$ & 5.338503 & 3.075316 & 2.911 \\
\hline $\mathrm{C}$ & 6.029593 & 2.084130 & 3.62 \\
\hline C & 5.346351 & 0.960628 & 4.14 \\
\hline C & 6.002040 & -0.269168 & 4.34 \\
\hline C & 5.295833 & -1.483137 & 4.22 \\
\hline $\mathrm{C}$ & 5.926190 & -2.665788 & $3.7 \varepsilon$ \\
\hline $\mathrm{C}$ & 5.197122 & -3.631219 & 3.05 \\
\hline $\mathrm{C}$ & 5.811752 & -4.410238 & 2.057 \\
\hline $\mathrm{C}$ & 5.066077 & -4.851738 & 0.944 \\
\hline $\mathrm{C}$ & 5.676681 & -4.974748 & -0.32 \\
\hline $\mathrm{C}$ & 4.928125 & -4.760514 & -1.49 \\
\hline $\mathrm{C}$ & 5.542271 & -4.216454 & -2.643 \\
\hline $\mathrm{C}$ & 4.803428 & -3.415172 & -3.5403 \\
\hline $\mathrm{C}$ & 5.423533 & -2.397135 & -4.291 \\
\hline $\mathrm{C}$ & 4.702961 & -1.243529 & -4.6 \\
\hline $\mathrm{C}$ & 5.734665 & 4.697456 & -0.41 \\
\hline $\mathrm{H}$ & 6.808740 & 4.652022 & -0.565 \\
\hline $\mathrm{C}$ & 5.452684 & 4.201744 & -2.75 \\
\hline $\mathrm{H}$ & 6.532744 & 4.180985 & -2.863 \\
\hline $\mathrm{C}$ & 5.333750 & -0.008979 & $-4 \cdot 9$ \\
\hline $\mathrm{H}$ & 6.415230 & 0.018906 & -4.996 \\
\hline $\mathrm{H}$ & -7.110908 & 2.042180 & -3.55 \\
\hline $\mathrm{H}$ & -7.047693 & 3.777575 & -1.797 \\
\hline $\mathrm{H}$ & -6.808359 & 4.652709 & 0.5661 \\
\hline $\mathrm{H}$ & -6.532252 & 4.181640 & 2.8 \\
\hline $\mathrm{H}$ & -7.085474 & -0.291301 & -4.2905 \\
\hline $\mathrm{H}$ & -6.389843 & 2.439802 & 4.49 \\
\hline $\mathrm{H}$ & -7.010244 & -2.684688 & -3.7293 \\
\hline $\mathrm{H}$ & -6.415178 & 0.018954 & 4.9960 \\
\hline $\mathrm{H}$ & -6.896122 & -4.414380 & -1.9 \\
\hline $\mathrm{H}$ & -6.507743 & -2.375187 & 4.345 \\
\hline I & -6.7 & 956250 & 0 \\
\hline
\end{tabular}




$\begin{array}{rrrr}\mathrm{H} & -6.627326 & -4.189850 & 2.691334 \\ \mathrm{H} & 6.895513 & -4.414447 & 1.992924 \\ \mathrm{H} & 6.760905 & -4.956565 & -0.380720 \\ \mathrm{H} & 7.009892 & -2.685399 & 3.728670 \\ \mathrm{H} & 6.626879 & -4.190781 & -2.692365 \\ \mathrm{H} & 7.085467 & -0.292450 & 4.290697 \\ \mathrm{H} & 6.507514 & -2.375639 & -4.346222 \\ \mathrm{H} & 7.111302 & 2.041236 & 3.551947 \\ \mathrm{H} & 7.048212 & 3.776855 & 1.797812 \\ \mathrm{H} & 6.390245 & 2.439180 & -4.489611 \\ \mathrm{O} & -1.027458 & 6.023465 & 0.813230 \\ \mathrm{O} & 1.027972 & 6.023758 & -0.812561\end{array}$

Nanotube-2O (Triplet) $\mathrm{ZPE}=1.189528 \quad \mathrm{Ee}=\quad-5651.862351$

$\begin{array}{lrrr}\text { C } & -5.799275 & 4.413864 & 2.057238 \\ \text { C } & -5.183340 & 3.650653 & 3.051891 \\ \text { C } & -5.923506 & 2.676190 & 3.780771 \\ \text { C } & -5.287803 & 1.493760 & 4.216077 \\ \text { C } & -6.003435 & 0.285818 & 4.313429 \\ \text { C } & -5.341013 & -0.955449 & 4.133819 \\ \text { C } & -6.035534 & -2.058364 & 3.594572 \\ \text { C } & -5.343454 & -3.066637 & 2.897317 \\ \text { C } & -5.985765 & -3.830231 & 1.883967 \\ \text { C } & -5.231349 & -4.414322 & 0.849125 \\ \text { C } & -5.755617 & -4.728580 & -0.418136 \\ \text { C } & -4.899439 & -4.659031 & -1.572448 \\ \text { C } & -5.454037 & -4.169224 & -2.751918 \\ \text { C } & -4.664542 & -3.374887 & -3.610979 \\ \text { C } & -5.303917 & -2.359744 & -4.364279 \\ \text { C } & -4.622539 & -1.190206 & -4.700422 \\ \text { C } & -5.320023 & 0.037957 & -4.909944 \\ \text { C } & -4.681920 & 1.266556 & -4.683567 \\ \text { C } & -5.405667 & 2.424155 & -4.315761 \\ \text { C } & -4.782478 & 3.444946 & -3.544468 \\ \text { C } & -5.522520 & 4.236866 & -2.660628 \\ \text { C } & -4.905834 & 4.780707 & -1.496130 \\ \text { C } & -5.657160 & 4.981351 & -0.324875 \\ \text { C } & -5.044582 & 4.868384 & 0.937634 \\ \text { C } & -3.741203 & 3.615839 & 3.118808 \\ \text { C } & -3.090877 & 2.595617 & 3.873976 \\ \text { C } & -3.844419 & 1.452621 & 4.266414 \\ \text { C } & -3.176499 & 0.213423 & 4.411338 \\ \text { C } & -3.907881 & -0.995512 & 4.203020 \\ \text { C } & -3.209770 & -2.152742 & 3.765049 \\ \text { C } & -3.914459 & -3.125773 & 3.017399 \\ \text { C } & -3.172428 & -3.991046 & 2.159308\end{array}$




\begin{tabular}{|c|c|c|c|}
\hline $\mathrm{C}$ & -3.803377 & -4.507658 & 1.01060 \\
\hline $\mathrm{C}$ & -2.998675 & -4.978726 & -0.06254 \\
\hline C & -3.464003 & -4.740614 & -1.42999 \\
\hline $\mathrm{C}$ & -2.618843 & -4.211163 & -2.45268 \\
\hline C & -3.242904 & -3.361273 & -3.4357 \\
\hline C & -2.506802 & -2.293303 & -4.04140 \\
\hline $\mathrm{C}$ & -3.193597 & -1.167263 & -4.54653 \\
\hline $\mathrm{C}$ & -2.504258 & 0.084504 & -4.65746 \\
\hline $\mathrm{C}$ & -3.237579 & 1.287952 & -4.57792 \\
\hline C & -2.577898 & 2.465796 & -4.12714 \\
\hline C & -3.339766 & 3.468688 & -3.462 \\
\hline C & -2.697240 & 4.277339 & -2.495 \\
\hline $\mathrm{C}$ & -3.473997 & 4.797724 & -1.41 \\
\hline C & -2.837368 & 4.991654 & -0.16 \\
\hline $\mathrm{C}$ & -3.614628 & 4.861876 & 1.02 \\
\hline C & -2.973906 & 4.367852 & 2.19 \\
\hline $\mathrm{C}$ & -1.672088 & 2.559766 & 3.93 \\
\hline C & -1.011186 & 1.375542 & 4.37 \\
\hline $\mathrm{C}$ & -1.750751 & 0.174586 & 4.46 \\
\hline C & -1.061720 & -1.070437 & 4.3 \\
\hline C & -1.772987 & -2.194896 & 3.82 \\
\hline $\mathrm{C}$ & -1.051189 & -3.247371 & 3.1 \\
\hline C & -1.760682 & -4.096288 & 2.25 \\
\hline $\mathrm{C}$ & -1.038668 & -4.928288 & 1.40 \\
\hline C & -1.703900 & -5.360938 & 0.2 \\
\hline $\mathrm{C}$ & -0.396201 & -5.056832 & $-1 \cdot 61$ \\
\hline $\mathrm{C}$ & -1.140356 & -4.225642 & -2.43 \\
\hline C & -0.394703 & -3.272039 & -3.23 \\
\hline C & -1.075509 & -2.248229 & -3.92 \\
\hline C & -0.364179 & -1.095458 & -4.3 \\
\hline C & -1.083894 & 0.114729 & -4.57 \\
\hline C & -0.406721 & 1.351292 & -4.43 \\
\hline C & -1.157567 & 2.498522 & -4.05 \\
\hline C & -0.501712 & 3.527980 & -3.32 \\
\hline C & -1.270481 & 4.303818 & -2.4 \\
\hline $\mathrm{C}$ & -0.627298 & 4.823712 & -1.2529 \\
\hline $\mathrm{C}$ & -1.405040 & 4.994146 & -0.0773 \\
\hline $\mathrm{C}$ & -0.766932 & 4.834461 & 1.1 \\
\hline C & -1.546743 & 4.345133 & 2.2759 \\
\hline C & -0.904241 & 3.551004 & 3.2 \\
\hline C & 0.417237 & 1.336871 & 4.4338 \\
\hline $\mathrm{C}$ & 1.086524 & 0.097311 & 4.57 \\
\hline C & 0.359175 & -1.109123 & 4.3496 \\
\hline C & 1.063116 & -2.264510 & 3.9193 \\
\hline C & 0.375717 & -3.282186 & 3.22 \\
\hline C & 1.114504 & -4.238551 & $2.420^{\circ}$ \\
\hline 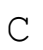 & 0.364286 & -5.064522 & 1 \\
\hline
\end{tabular}




\begin{tabular}{|c|c|c|c|}
\hline C & 1.669616 & -5.370710 & -0.24660 \\
\hline $\mathrm{C}$ & 1.007783 & -4.931302 & -1.41812 \\
\hline C & 1.735216 & -4.100834 & -2.30616 \\
\hline $\mathrm{C}$ & 1.031882 & -3.246129 & -3.19732 \\
\hline $\mathrm{C}$ & 1.761092 & -2.195194 & -3.8316 \\
\hline $\mathrm{C}$ & 1.057652 & -1.065509 & $-4.3037 s$ \\
\hline $\mathrm{C}$ & 1.754331 & 0.175536 & -4.4628 \\
\hline C & 1.020744 & 1.381596 & -4.37187 \\
\hline $\mathrm{C}$ & 1.687627 & 2.560043 & -3.9289 \\
\hline C & 0.925367 & 3.554192 & -3.2492 \\
\hline $\mathrm{C}$ & 1.572354 & 4.343918 & -2.26542 \\
\hline $\mathrm{C}$ & 0.794867 & 4.832713 & -1.17144 \\
\hline $\mathrm{C}$ & 1.433342 & 4.987891 & 0.0885 \\
\hline $\mathrm{C}$ & 0.653955 & 4.818343 & 1.26456 \\
\hline $\mathrm{C}$ & 1.294869 & 4.290659 & 2.4284 \\
\hline $\mathrm{C}$ & 0.523359 & 3.516904 & 3.3272 \\
\hline $\mathrm{C}$ & 1.174255 & 2.482104 & 4.06374 \\
\hline $\mathrm{C}$ & 2.507248 & 0.058363 & 4.6532 \\
\hline $\mathrm{C}$ & 3.189615 & -1.195893 & 4.54029 \\
\hline $\mathrm{C}$ & 2.495003 & -2.319036 & 4.031 \\
\hline $\mathrm{C}$ & 3.222869 & -3.387496 & 3.42621 \\
\hline $\mathrm{C}$ & 2.592350 & -4.234109 & 2.4 \\
\hline $\mathrm{C}$ & 3.432211 & -4.765341 & 1.417 \\
\hline C & 2.966851 & -4.999708 & 0.04912 \\
\hline $\mathrm{C}$ & 3.773785 & -4.527949 & -1.02225 \\
\hline $\mathrm{C}$ & 3.147757 & -4.004674 & -2.16798 \\
\hline $\mathrm{C}$ & 3.896974 & -3.140184 & -3.0 \\
\hline $\mathrm{C}$ & 3.197911 & -2.161261 & -3.7686 \\
\hline $\mathrm{C}$ & 3.904002 & -1.006444 & -4.2012 \\
\hline C & 3.179017 & 0.206387 & -4.40 \\
\hline $\mathrm{C}$ & 3.854916 & 1.442404 & -4.2604 \\
\hline $\mathrm{C}$ & 3.107864 & 2.587838 & -3.8650 \\
\hline $\mathrm{C}$ & 3.763174 & 3.603864 & -3.1099 \\
\hline $\mathrm{C}$ & 2.998501 & 4.359477 & -2.1886 \\
\hline $\mathrm{C}$ & 3.640212 & 4.846892 & -1.00872 \\
\hline $\mathrm{C}$ & 2.864869 & 4.979134 & 0.1722 \\
\hline $\mathrm{C}$ & 3.500666 & 4.778910 & 1.4272 \\
\hline $\mathrm{C}$ & 2.722810 & 4.257222 & 2.5045 \\
\hline C & 3.361235 & 3.442766 & 3.4694 \\
\hline $\mathrm{C}$ & 2.593458 & 2.441363 & 4.1312 \\
\hline $\mathrm{C}$ & 3.247509 & 1.258211 & 4.5771 \\
\hline $\mathrm{C}$ & 4.616473 & -1.226940 & 4.6887 \\
\hline $\mathrm{C}$ & 5.290927 & -2.402863 & 4.3493 \\
\hline $\mathrm{C}$ & 4.644931 & -3.412402 & 3.6025 \\
\hline $\mathrm{C}$ & 4.871909 & -4.699163 & 1.5628 \\
\hline $\mathrm{C}$ & 5.204309 & -4.446894 & -0.8615 \\
\hline $\mathrm{C}$ & 5.959886 & -3.864336 & -1.879 \\
\hline
\end{tabular}




\begin{tabular}{|c|c|c|c|}
\hline $\mathrm{C}$ & 5.322215 & -3.086596 & -2.899087 \\
\hline C & 6.019871 & -2.077343 & -3.577219 \\
\hline C & 5.334356 & -0.973673 & -4.122548 \\
\hline $\mathrm{C}$ & 6.004820 & 0.269472 & -4.291217 \\
\hline C & 5.296616 & 1.473202 & -4.203700 \\
\hline C & 5.938581 & 2.660966 & -3.759115 \\
\hline C & 5.204631 & 3.632261 & -3.044543 \\
\hline C & 5.825323 & 4.409216 & -2.045671 \\
\hline C & 5.074491 & 4.853739 & -0.927882 \\
\hline C & 5.687416 & 4.974175 & 0.336507 \\
\hline C & 4.933913 & 4.759252 & 1.510390 \\
\hline $\mathrm{C}$ & 5.547583 & 4.211670 & 2.665852 \\
\hline C & 4.802779 & 3.409934 & 3.550908 \\
\hline C & 5.421626 & 2.386257 & 4.306315 \\
\hline C & 4.690855 & 1.226589 & 4.676800 \\
\hline $\mathrm{C}$ & 5.725855 & -4.787819 & 0.412532 \\
\hline $\mathrm{H}$ & 6.799999 & -4.781269 & 0.566706 \\
\hline C & 5.428791 & -4.221302 & 2.745468 \\
\hline $\mathrm{H}$ & 6.508683 & -4.202199 & 2.854894 \\
\hline C & 5.322554 & -0.002897 & 4.890432 \\
\hline $\mathrm{H}$ & 6.404668 & -0.036833 & 4.97 \\
\hline $\mathrm{H}$ & -7.116975 & -2.006533 & 3.511217 \\
\hline $\mathrm{H}$ & -7.065481 & -3.770931 & 1.787967 \\
\hline $\mathrm{H}$ & -6.829315 & -4.702796 & -0.574175 \\
\hline $\mathrm{H}$ & -6.534058 & -4.136236 & -2.857075 \\
\hline $\mathrm{H}$ & -7.087532 & 0.311123 & 4.257972 \\
\hline $\mathrm{H}$ & -6.385454 & -2.389054 & -4.455788 \\
\hline $\mathrm{H}$ & -7.007921 & 2.704827 & 3.737639 \\
\hline $\mathrm{H}$ & -6.401453 & 0.010963 & -5.003183 \\
\hline $\mathrm{H}$ & -6.882954 & 4.418342 & 1.989502 \\
\hline $\mathrm{H}$ & -6.488820 & 2.412708 & -4.389615 \\
\hline $\mathrm{H}$ & -6.740956 & 4.950960 & -0.385047 \\
\hline $\mathrm{H}$ & -6.606974 & 4.214630 & -2.710990 \\
\hline $\mathrm{H}$ & 6.909276 & 4.415468 & -1.983359 \\
\hline $\mathrm{H}$ & 6.771326 & 4.951773 & 0.397493 \\
\hline $\mathrm{H}$ & 7.023025 & 2.680501 & -3.710867 \\
\hline $\mathrm{H}$ & 6.631881 & 4.186018 & 2.718130 \\
\hline $\mathrm{H}$ & 7.088414 & 0.288082 & -4.224370 \\
\hline $\mathrm{H}$ & 6.505075 & 2.365493 & 4.374056 \\
\hline $\mathrm{H}$ & 7.100345 & -2.026402 & -3.481520 \\
\hline $\mathrm{H}$ & 7.039841 & -3.810481 & -1.783001 \\
\hline $\mathrm{H}$ & 6.372556 & -2.437265 & 4.437849 \\
\hline O & -1.039214 & -6.019202 & -0.822541 \\
\hline O & 1.001928 & -6.029685 & 0.806522 \\
\hline
\end{tabular}

Nanotube-2O (Quintet) $\mathrm{ZPE}=\quad 1.189901 \quad \mathrm{Ee}=\quad-5651.871980$ 


\begin{tabular}{|c|c|c|c|}
\hline C & -5.803670 & 4.403611 & 2.04726 \\
\hline C & -5.187724 & 3.647815 & 3.0461 \\
\hline C & -5.930065 & 2.667189 & 3.7734 \\
\hline $\mathrm{C}$ & -5.295425 & 1.489874 & 4.2073 \\
\hline $\mathrm{C}$ & -6.009647 & 0.278649 & 4.3121 \\
\hline C & -5.344903 & -0.961964 & 4.1269 \\
\hline C & -6.036206 & -2.063814 & 3.5869 \\
\hline C & -5.341032 & -3.073656 & 2.8916 \\
\hline $\mathrm{C}$ & -5.981676 & -3.838545 & 1.8769 \\
\hline C & -5.226247 & -4.422863 & 0.8450 \\
\hline C & -5.749986 & -4.749222 & -0.4208 \\
\hline $\mathrm{C}$ & -4.892147 & -4.669864 & -1.5767 \\
\hline $\mathrm{C}$ & -5.446394 & -4.177059 & -2.7522 \\
\hline $\mathrm{C}$ & -4.656886 & -3.379687 & -3.6118 \\
\hline $\mathrm{C}$ & -5.298041 & -2.365995 & -4.3628 \\
\hline $\mathrm{C}$ & -4.617722 & -1.193483 & -4.699 \\
\hline $\mathrm{C}$ & -5.316462 & 0.032189 & -4.9066 \\
\hline C & -4.678549 & 1.262247 & -4.6843 \\
\hline $\mathrm{C}$ & -5.404303 & 2.419877 & -4.3147 \\
\hline $\mathrm{C}$ & -4.782907 & 3.440952 & -3.5488 \\
\hline $\mathrm{C}$ & -5.524887 & 4.238643 & -2.6672 \\
\hline $\mathrm{C}$ & -4.910203 & 4.778866 & -1.5 \\
\hline $\mathrm{C}$ & -5.661579 & 4.980642 & -0.3323 \\
\hline C & -5.048837 & 4.865544 & 0.9346 \\
\hline $\mathrm{C}$ & -3.749112 & 3.615880 & 3.11 \\
\hline $\mathrm{C}$ & -3.097708 & 2.592128 & 3.8711 \\
\hline $\mathrm{C}$ & -3.849146 & 1.450013 & 4.25 \\
\hline $\mathrm{C}$ & -3.180056 & 0.211355 & 4.4087 \\
\hline $\mathrm{C}$ & -3.910765 & -1.000357 & 4.1970 \\
\hline C & -3.211686 & -2.153621 & 3.76 \\
\hline $\mathrm{C}$ & -3.914922 & -3.130601 & 3.0133 \\
\hline $\mathrm{C}$ & -3.169722 & -3.992591 & $2.153^{\prime}$ \\
\hline $\mathrm{C}$ & -3.797258 & -4.509967 & 1.0068 \\
\hline $\mathrm{C}$ & -2.990441 & -4.986811 & -0.0650 \\
\hline $\mathrm{C}$ & -3.456336 & -4.747156 & -1.432 \\
\hline $\mathrm{C}$ & -2.611513 & -4.213665 & -2.4523 \\
\hline $\mathrm{C}$ & -3.236140 & -3.364146 & -3.4362 \\
\hline $\mathrm{C}$ & -2.500501 & -2.295542 & -4.0409 \\
\hline C & -3.189027 & -1.169168 & -4.5460 \\
\hline $\mathrm{C}$ & -2.501097 & 0.081960 & -4.6570 \\
\hline $\mathrm{C}$ & -3.236410 & 1.285433 & -4.5794 \\
\hline $\mathrm{C}$ & -2.577751 & 2.464458 & -4.1266 \\
\hline C & -3.340005 & 3.465606 & -3.4651 \\
\hline $\mathrm{C}$ & -2.697845 & 4.278439 & -2.4980 \\
\hline $\mathrm{C}$ & -3.475421 & 4.795604 & -1.4167 \\
\hline $\mathrm{C}$ & -2.840448 & 4.992824 & -0.1652 \\
\hline $\mathrm{C}$ & -3.620499 & 4.861147 & 1.01 \\
\hline
\end{tabular}




\begin{tabular}{|c|c|c|c|}
\hline $\mathrm{C}$ & -2.981227 & 4.365664 & 2.1955 \\
\hline $\mathrm{C}$ & -1.678606 & 2.557931 & 3.93513 \\
\hline C & -1.017756 & 1.376756 & 4.37204 \\
\hline $\mathrm{C}$ & -1.757420 & 0.173603 & 4.461 \\
\hline C & -1.066058 & -1.070879 & 4.297 \\
\hline C & -1.774016 & -2.194316 & 3.822 \\
\hline $\mathrm{C}$ & -1.049552 & -3.248562 & 3.186 \\
\hline $\mathrm{C}$ & -1.756996 & -4.098593 & 2.2924 \\
\hline $\mathrm{C}$ & -1.034326 & -4.931692 & 1.404 \\
\hline C & -1.698320 & -5.369200 & 0.230 \\
\hline C & -0.388259 & -5.060065 & -1.61 \\
\hline C & -1.133208 & -4.224811 & $-2 \cdot 4$ \\
\hline $\mathrm{C}$ & -0.389160 & -3.271777 & -3.23 \\
\hline $\mathrm{C}$ & -1.071040 & -2.249107 & -3.92 \\
\hline $\mathrm{C}$ & -0.360556 & -1.094393 & -4.35 \\
\hline C & -1.080690 & 0.113246 & -4.57 \\
\hline $\mathrm{C}$ & -0.403671 & 1.352168 & -4.4 \\
\hline $\mathrm{C}$ & -1.156072 & 2.498499 & -4.05 \\
\hline $\mathrm{C}$ & -0.501655 & 3.528193 & -3.322 \\
\hline C & -1.273156 & 4.305855 & -2.4 \\
\hline C & -0.631530 & 4.823433 & -1.25 \\
\hline $\mathrm{C}$ & -1.410130 & 4.996109 & -0.07 \\
\hline C & -0.772356 & 4.835264 & 1.1 \\
\hline $\mathrm{C}$ & -1.551624 & 4.344172 & 2.27 \\
\hline C & -0.909564 & 3.553290 & 3.2 \\
\hline $\mathrm{C}$ & 0.411187 & 1.339697 & 4.4 \\
\hline $\mathrm{C}$ & 1.082194 & 0.098390 & $4.5^{\circ}$ \\
\hline C & 0.356184 & -1.107572 & 4.34 \\
\hline C & 1.062003 & -2.261753 & 3.9 \\
\hline C & 0.375033 & -3.282407 & 3.2 \\
\hline $\mathrm{C}$ & 1.116612 & -4.235585 & 2.42 \\
\hline C & 0.368197 & -5.065741 & 1.60 \\
\hline C & 1.677503 & -5.370840 & -0.2 \\
\hline C & 1.014630 & -4.930780 & -1.4 \\
\hline C & 1.741857 & -4.098703 & $-2 \cdot 3$ \\
\hline $\mathrm{C}$ & 1.038091 & -3.244437 & -3.194 \\
\hline C & 1.767532 & -2.194331 & -3.8316 \\
\hline C & 1.061989 & -1.062669 & -4.30 \\
\hline C & 1.756761 & 0.175789 & -4.4613 \\
\hline C & 1.021139 & 1.383892 & -4.3 \\
\hline C & 1.686310 & 2.561583 & -3.9255 \\
\hline $\mathrm{C}$ & 0.924122 & 3.555185 & -3.25 \\
\hline C & 1.570910 & 4.348277 & -2.26 \\
\hline $\mathrm{C}$ & 0.792808 & 4.833315 & -1.1708 \\
\hline C & 1.430426 & 4.992905 & 0.08 \\
\hline C & 0.648232 & 4.819975 & 1.2 \\
\hline$C$ & $1.287^{\circ}$ & 4.293226 & 2 \\
\hline
\end{tabular}




\begin{tabular}{|c|c|c|c|}
\hline $\mathrm{C}$ & 0.514900 & 3.519861 & 3.32944 \\
\hline $\mathrm{C}$ & 1.165988 & 2.482811 & 4.06422 \\
\hline $\mathrm{C}$ & 2.500593 & 0.059569 & 4.6582 \\
\hline $\mathrm{C}$ & 3.183663 & -1.190512 & 4.54206 \\
\hline $\mathrm{C}$ & 2.491064 & -2.314831 & 4.0345 \\
\hline $\mathrm{C}$ & 3.221144 & -3.383685 & 3.42506 \\
\hline $\mathrm{C}$ & 2.594529 & -4.228432 & 2.443 \\
\hline $\mathrm{C}$ & 3.439085 & -4.758737 & 1.4161 \\
\hline $\mathrm{C}$ & 2.973711 & -4.991788 & 0.052 \\
\hline $\mathrm{C}$ & 3.783898 & -4.527734 & -1.022 \\
\hline $\mathrm{C}$ & 3.155441 & -4.002070 & -2.1700 \\
\hline $\mathrm{C}$ & 3.901206 & -3.139465 & -3.0 \\
\hline C & 3.201135 & -2.159931 & -3.7703 \\
\hline C & 3.905334 & -1.001249 & -4.1998 \\
\hline $\mathrm{C}$ & 3.181624 & 0.206792 & -4.408 \\
\hline $\mathrm{C}$ & 3.857802 & 1.447890 & -4.260 \\
\hline $\mathrm{C}$ & 3.109439 & 2.590113 & -3.8605 \\
\hline C & 3.763296 & 3.606652 & -3.1095 \\
\hline C & 2.995230 & 4.364275 & -2.184 \\
\hline C & 3.635142 & 4.846688 & -1.00 \\
\hline $\mathrm{C}$ & 2.858272 & 4.984399 & 0.17 \\
\hline $\mathrm{C}$ & 3.493863 & 4.780125 & 1.4 \\
\hline $\mathrm{C}$ & 2.716667 & 4.261089 & 2.50 \\
\hline $\mathrm{C}$ & 3.355708 & 3.447665 & 3.4761 \\
\hline $\mathrm{C}$ & 2.587536 & 2.443329 & 4.13 \\
\hline $\mathrm{C}$ & 3.241757 & 1.264803 & 4.582 \\
\hline $\mathrm{C}$ & 4.614333 & -1.221240 & 4.6996 \\
\hline $\mathrm{C}$ & 5.288443 & -2.405787 & 4.3729 \\
\hline $\mathrm{C}$ & 4.646877 & -3.410011 & 3.6079 \\
\hline $\mathrm{C}$ & 4.875968 & -4.685397 & 1.5 \\
\hline $\mathrm{C}$ & 5.207879 & -4.437891 & -0.8571 \\
\hline $\mathrm{C}$ & 5.966676 & -3.849622 & -1.8849 \\
\hline $\mathrm{C}$ & 5.330765 & -3.085668 & -2.898 \\
\hline $\mathrm{C}$ & 6.029509 & -2.078282 & -3.5940 \\
\hline $\mathrm{C}$ & 5.341228 & -0.967999 & -4.12 \\
\hline $\mathrm{C}$ & 6.008252 & 0.268594 & -4.299 \\
\hline $\mathrm{C}$ & 5.296088 & 1.480707 & -4.2037 \\
\hline $\mathrm{C}$ & 5.936591 & 2.656900 & -3.7492 \\
\hline $\mathrm{C}$ & 5.201699 & 3.634264 & -3.0410 \\
\hline $\mathrm{C}$ & 5.822579 & 4.409885 & -2.0 \\
\hline $\mathrm{C}$ & 5.071960 & 4.853589 & -0.9235 \\
\hline $\mathrm{C}$ & 5.683271 & 4.978499 & 0.340 \\
\hline $\mathrm{C}$ & 4.928034 & 4.759290 & 1.5158 \\
\hline $\mathrm{C}$ & 5.540054 & 4.208998 & 2.6667 \\
\hline $\mathrm{C}$ & 4.792597 & 3.412507 & 3.5 \\
\hline $\mathrm{C}$ & 5.412096 & 2.380359 & 4.30 \\
\hline $\mathrm{C}$ & 582 & 76 & 4 \\
\hline
\end{tabular}




$\begin{array}{lrrr}\mathrm{C} & 5.729120 & -4.745476 & 0.417935 \\ \mathrm{H} & 6.802633 & -4.713423 & 0.574806 \\ \mathrm{C} & 5.430378 & -4.213201 & 2.758099 \\ \mathrm{H} & 6.510100 & -4.195734 & 2.869480 \\ \mathrm{C} & 5.315575 & -0.005964 & 4.906845 \\ \mathrm{H} & 6.397001 & -0.037024 & 4.999200 \\ \mathrm{H} & -7.117627 & -2.014514 & 3.501822 \\ \mathrm{H} & -7.061592 & -3.782155 & 1.781454 \\ \mathrm{H} & -6.823784 & -4.734180 & -0.577173 \\ \mathrm{H} & -6.526367 & -4.143586 & -2.858302 \\ \mathrm{H} & -7.093977 & 0.302253 & 4.261611 \\ \mathrm{H} & -6.379498 & -2.396912 & -4.454757 \\ \mathrm{H} & -7.014349 & 2.696654 & 3.727115 \\ \mathrm{H} & -6.398018 & 0.003856 & -4.998258 \\ \mathrm{H} & -6.887127 & 4.401208 & 1.975145 \\ \mathrm{H} & -6.487540 & 2.405702 & -4.387526 \\ \mathrm{H} & -6.745415 & 4.951167 & -0.392148 \\ \mathrm{H} & -6.609203 & 4.218869 & -2.720769 \\ \mathrm{H} & 6.906480 & 4.414092 & -1.975615 \\ \mathrm{H} & 6.767094 & 4.958430 & 0.403530 \\ \mathrm{H} & 7.020814 & 2.673676 & -3.693275 \\ \mathrm{H} & 6.624310 & 4.177770 & 2.717330 \\ \mathrm{H} & 7.092107 & 0.289816 & -4.237456 \\ \mathrm{H} & 6.495995 & 2.357473 & 4.366912 \\ \mathrm{H} & 7.111094 & -2.032940 & -3.509230 \\ \mathrm{H} & 7.045919 & -3.790528 & -1.783292 \\ \mathrm{H} & 6.368621 & -2.446005 & 4.475243 \\ \mathrm{O} & -1.029438 & -6.023894 & -0.824238 \\ \mathrm{O} & 1.010580 & -6.030497 & 0.808053\end{array}$

SW Defected Nanotube-2O (Singlet) $\mathrm{ZPE}=1.189013 \quad \mathrm{Ee}=\quad-5651.718293$

\begin{tabular}{|c|c|c|c|}
\hline C & -5.123384 & -5.551006 & -0.405855 \\
\hline $\mathrm{C}$ & -5.453339 & -4.863887 & 1.926879 \\
\hline C & -5.830532 & -3.049535 & 3.550755 \\
\hline $\mathrm{C}$ & -6.169585 & -0.660352 & 4.037934 \\
\hline C & -6.439022 & 1.652257 & 3.304404 \\
\hline C & -5.271885 & 1.638906 & -4.533426 \\
\hline C & -4.928220 & -4.892967 & -2.756422 \\
\hline $\mathrm{C}$ & -4.332606 & -5.316138 & -1.549539 \\
\hline $\mathrm{C}$ & -4.607040 & -5.285841 & 0.879859 \\
\hline $\mathrm{C}$ & -4.975563 & -3.973100 & 2.908809 \\
\hline $\mathrm{C}$ & -5.339275 & -1.805075 & 3.981158 \\
\hline $\mathrm{C}$ & -5.639786 & 0.625518 & 3.875294 \\
\hline $\mathrm{C}$ & -5.846681 & 2.750923 & 2.674770 \\
\hline $\mathrm{C}$ & -5.721314 & 4.181055 & 0.6399 \\
\hline $\mathrm{C}$ & -5.201101 & 4.146174 & -1.854600 \\
\hline
\end{tabular}




\begin{tabular}{|c|c|c|c|}
\hline $\mathrm{C}$ & -4.765335 & 2.699561 & -3.75945 \\
\hline $\mathrm{C}$ & -4.470013 & 0.536359 & -4.84101 \\
\hline $\mathrm{C}$ & -4.282862 & -1.901273 & -4.81789 \\
\hline $\mathrm{C}$ & -4.223666 & -4.049949 & -3.64167 \\
\hline $\mathrm{C}$ & -2.903162 & -5.178867 & -1.3939 \\
\hline $\mathrm{C}$ & -3.179178 & -5.124529 & 1.0271 \\
\hline $\mathrm{C}$ & -3.549615 & -3.790246 & 3.0328 \\
\hline $\mathrm{C}$ & -3.913319 & -1.612955 & 4.0756 \\
\hline $\mathrm{C}$ & -4.202281 & 0.814979 & 3.9924 \\
\hline $\mathrm{C}$ & -4.430338 & 2.972848 & 2.9331 \\
\hline $\mathrm{C}$ & -4.394090 & 4.546455 & 1.055 \\
\hline $\mathrm{C}$ & -3.798625 & 4.507794 & -1.85 \\
\hline $\mathrm{C}$ & -3.345315 & 2.850803 & -3.553 \\
\hline $\mathrm{C}$ & -3.051241 & 0.664240 & -4.6287 \\
\hline $\mathrm{C}$ & -2.854106 & -1.774888 & -4.64 \\
\hline $\mathrm{C}$ & -2.792503 & -3.926253 & -3.484 \\
\hline $\mathrm{C}$ & -2.125861 & -4.633743 & -2.45 \\
\hline $\mathrm{C}$ & -2.322900 & -5.253021 & -0.102 \\
\hline $\mathrm{C}$ & -2.657185 & -4.518730 & 2.1 \\
\hline $\mathrm{C}$ & -3.037895 & -2.681409 & 3.75 \\
\hline $\mathrm{C}$ & -3.370514 & -0.302249 & 4.21 \\
\hline $\mathrm{C}$ & -3.605088 & 2.054504 & 3.6 \\
\hline $\mathrm{C}$ & -3.788797 & 3.958887 & 2.2257 \\
\hline $\mathrm{C}$ & -3.408651 & 5.155558 & 0.3 \\
\hline $\mathrm{C}$ & -2.831850 & 3.889917 & -2.6652 \\
\hline $\mathrm{C}$ & -2.490017 & 1.873217 & -4.12 \\
\hline $\mathrm{C}$ & -2.238285 & -0.501825 & -4.7 \\
\hline $\mathrm{C}$ & -2.103789 & -2.868916 & -4.1317 \\
\hline $\mathrm{C}$ & -0.710305 & -4.505070 & $-2.299^{\circ}$ \\
\hline $\mathrm{C}$ & -0.907708 & -5.106300 & 0.0 \\
\hline $\mathrm{C}$ & -1.244046 & -4.356558 & 2.3506 \\
\hline $\mathrm{C}$ & -1.626583 & -2.504950 & 3.89 \\
\hline $\mathrm{C}$ & -1.955716 & -0.113319 & 4.31 \\
\hline $\mathrm{C}$ & -2.173419 & 2.249649 & 3.649 \\
\hline $\mathrm{C}$ & -2.395331 & 4.177085 & 2.2339 \\
\hline $\mathrm{C}$ & -0.983610 & 5.222154 & 0.4467 \\
\hline $\mathrm{C}$ & -1.336207 & 4.057323 & -2.6392 \\
\hline $\mathrm{C}$ & -1.056613 & 1.998277 & -4.0348 \\
\hline $\mathrm{C}$ & -0.822780 & -0.384590 & -4.5799 \\
\hline $\mathrm{C}$ & -0.689776 & -2.750468 & -3.9934 \\
\hline $\mathrm{C}$ & 0.024049 & -3.682546 & -3.1957 \\
\hline $\mathrm{C}$ & -0.084957 & -4.903063 & -1.0846 \\
\hline $\mathrm{C}$ & -0.361284 & -4.815735 & 1.3379 \\
\hline $\mathrm{C}$ & -0.736666 & -3.453236 & 3.3253 \\
\hline $\mathrm{C}$ & -1.106137 & -1.252339 & 4.3123 \\
\hline $\mathrm{C}$ & -1.377622 & 1.174977 & 4.1342 \\
\hline $\mathrm{C}$ & -1.523101 & 3.361534 & 2.97 \\
\hline
\end{tabular}




\begin{tabular}{|c|c|c|c|}
\hline $\mathrm{C}$ & -2.155684 & 4.998761 & 1.055975 \\
\hline $\mathrm{C}$ & -0.609464 & 4.877318 & -1.788585 \\
\hline $\mathrm{C}$ & -0.487159 & 3.135478 & -3.408622 \\
\hline $\mathrm{C}$ & -0.228898 & 0.899585 & -4.401253 \\
\hline C & -0.038867 & -1.545144 & -4.379498 \\
\hline $\mathrm{C}$ & 1.439877 & -3.560071 & -3.055194 \\
\hline $\mathrm{C}$ & 1.328653 & -4.756130 & -0.929253 \\
\hline $\mathrm{C}$ & 1.050452 & -4.649937 & 1.492872 \\
\hline C & 0.670593 & -3.282676 & 3.475926 \\
\hline $\mathrm{C}$ & 0.295605 & -1.081998 & 4.458766 \\
\hline C & 0.033845 & 1.345659 & 4.251214 \\
\hline $\mathrm{C}$ & -0.045283 & 3.482262 & 2.977816 \\
\hline $\mathrm{C}$ & 0.347925 & 4.905836 & 0.842705 \\
\hline $\mathrm{C}$ & 0.776832 & 4.793296 & -1.575503 \\
\hline $\mathrm{C}$ & 0.950871 & 3.225745 & -3.378438 \\
\hline $\mathrm{C}$ & 1.195484 & 0.999145 & -4.347602 \\
\hline $\mathrm{C}$ & 1.380685 & -1.441079 & -4.273750 \\
\hline C & 2.131529 & -2.527587 & -3.743424 \\
\hline C & 2.109119 & -4.237468 & -2.001091 \\
\hline $\mathrm{C}$ & 1.909864 & -4.791664 & 0.366315 \\
\hline C & 1.568314 & -4.017839 & 2.653741 \\
\hline $\mathrm{C}$ & 1.172562 & -2.163614 & 4.188807 \\
\hline C & 0.838237 & 0.227352 & 4.579944 \\
\hline $\mathrm{C}$ & 0.666367 & 2.558294 & 3.806407 \\
\hline C & 0.772168 & 4.315493 & 2.090521 \\
\hline $\mathrm{C}$ & 1.265047 & 5.021578 & -0.234912 \\
\hline $\mathrm{C}$ & 1.588918 & 4.120518 & -2.474629 \\
\hline C & 1.788487 & 2.215271 & -3.941263 \\
\hline $\mathrm{C}$ & 2.003713 & -0.178319 & -4.413410 \\
\hline $\mathrm{C}$ & 3.548958 & -2.420838 & -3.623289 \\
\hline $\mathrm{C}$ & 3.524146 & -4.100024 & -1.853269 \\
\hline C & 3.323190 & -4.623625 & 0.519901 \\
\hline C & 2.980503 & -3.838491 & 2.803466 \\
\hline C & 2.582801 & -1.997275 & 4.354176 \\
\hline C & 2.242547 & 0.379410 & 4.794806 \\
\hline $\mathrm{C}$ & 2.076344 & 2.701502 & 4.058525 \\
\hline $\mathrm{C}$ & 2.206054 & 4.387368 & 2.319178 \\
\hline C & 2.649023 & 4.964028 & -0.010998 \\
\hline $\mathrm{C}$ & 3.005105 & 4.149003 & -2.275431 \\
\hline $\mathrm{C}$ & 3.228337 & 2.305943 & -3.838082 \\
\hline $\mathrm{C}$ & 3.428216 & -0.082932 & -4.327552 \\
\hline C & 4.203851 & -1.241791 & -4.074377 \\
\hline $\mathrm{C}$ & 4.256446 & -3.321285 & -2.786626 \\
\hline $\mathrm{C}$ & 4.142778 & -4.451902 & -0.623893 \\
\hline $\mathrm{C}$ & 3.863999 & -4.297917 & 1.795836 \\
\hline C & 3.482144 & -2.932892 & 3.781747 \\
\hline $\mathrm{C}$ & 3.093928 & -0.761135 & 4.825979 \\
\hline
\end{tabular}




\begin{tabular}{|c|c|c|c|}
\hline $\mathrm{C}$ & 2.817478 & 1.661653 & 4.70192 \\
\hline C & 2.800388 & 3.732681 & 3.43168 \\
\hline $\mathrm{C}$ & 3.108182 & 4.861877 & 1.31452 \\
\hline $\mathrm{C}$ & 3.537950 & 4.718348 & -1.09729 \\
\hline C & 3.839617 & 3.355420 & -3.0926 \\
\hline C & 4.042091 & 1.195243 & -4.1752 \\
\hline $\mathrm{C}$ & 5.642754 & -1.138579 & -3.9807 \\
\hline $\mathrm{C}$ & 5.687864 & -3.195247 & -2.6497 \\
\hline $\mathrm{C}$ & 5.569611 & -4.294051 & -0.46325 \\
\hline C & 5.290286 & -4.125967 & 1.9642 \\
\hline C & 4.906519 & -2.768963 & 3.967 \\
\hline C & 4.510553 & -0.611574 & 5.057 \\
\hline $\mathrm{C}$ & 4.224804 & 1.817854 & 4.986 \\
\hline C & 4.215099 & 3.890864 & 3.704 \\
\hline $\mathrm{C}$ & 4.535192 & 4.944377 & 1.53 \\
\hline C & 4.960851 & 4.753666 & -0.8848 \\
\hline $\mathrm{C}$ & 5.273293 & 3.438971 & -2.9 \\
\hline C & 5.485149 & 1.299855 & -4.08 \\
\hline $\mathrm{C}$ & 6.323896 & -3.824746 & -1.561 \\
\hline C & 6.119306 & -4.303992 & 0.83 \\
\hline $\mathrm{C}$ & 5.773298 & -3.517689 & 3.13 \\
\hline $\mathrm{C}$ & 5.362871 & -1.696136 & 4.7 \\
\hline C & 4.998882 & 0.687623 & 5.3 \\
\hline $\mathrm{C}$ & 4.840010 & 3.033431 & 4.62 \\
\hline C & 5.015671 & 4.638975 & 2.8 \\
\hline C & 5.407946 & 5.046411 & 0.42 \\
\hline C & 5.796103 & 4.262925 & -1.9059 \\
\hline C & 6.063362 & 2.495446 & -3.6160 \\
\hline $\mathrm{H}$ & -6.347427 & 1.558629 & -4.652 \\
\hline $\mathrm{H}$ & -6.525905 & -4.980118 & 1.8 \\
\hline $\mathrm{H}$ & -6.198078 & -5.648360 & -0.5257 \\
\hline $\mathrm{H}$ & -6.004762 & -4.983231 & -2.866 \\
\hline $\mathrm{H}$ & -6.903346 & -3.177079 & 3.44 \\
\hline $\mathrm{H}$ & -7.241147 & -0.796463 & 3.9306 \\
\hline $\mathrm{H}$ & -7.495270 & 1.452153 & 3.1 \\
\hline $\mathrm{H}$ & 6.844138 & -3.380912 & 3.25 \\
\hline $\mathrm{H}$ & 7.190563 & -4.168003 & 0.94 \\
\hline $\mathrm{H}$ & 7.397514 & -3.708283 & -1.445 \\
\hline $\mathrm{H}$ & 7.141941 & 2.540051 & -3.5072 \\
\hline $\mathrm{H}$ & 6.431814 & -1.567383 & 4.8 \\
\hline $\mathrm{H}$ & 6.870906 & 4.280402 & -1.7585 \\
\hline $\mathrm{H}$ & 6.061661 & 0.820923 & $5.4 \varepsilon$ \\
\hline $\mathrm{H}$ & 6.475952 & 5.086106 & 0.6175 \\
\hline $\mathrm{H}$ & 5.900746 & 3.160260 & 4.8195 \\
\hline $\mathrm{H}$ & 6.079295 & 4.721602 & 3.0 \\
\hline C & -6.471172 & 3.436334 & 1.5 \\
\hline 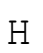 & -7.4 & 3.168680 & 1 \\
\hline
\end{tabular}




$\begin{array}{lrrr}\mathrm{C} & -6.089427 & 4.237566 & -0.713950 \\ \mathrm{H} & -7.116499 & 3.957710 & -0.938276 \\ \mathrm{C} & -5.663395 & 3.409120 & -2.936691 \\ \mathrm{H} & -6.719773 & 3.172111 & -3.006188 \\ \mathrm{C} & -5.026877 & -0.742209 & -5.081591 \\ \mathrm{H} & -6.101531 & -0.821972 & -5.210745 \\ \mathrm{C} & -4.902325 & -3.118353 & -4.451018 \\ \mathrm{H} & -5.978036 & -3.210271 & -4.566064 \\ \mathrm{C} & 6.249182 & 0.116235 & -4.171098 \\ \mathrm{H} & 7.328261 & 0.191509 & -4.081498 \\ \mathrm{C} & 6.358262 & -2.206339 & -3.399375 \\ \mathrm{H} & 7.435699 & -2.115563 & -3.298512 \\ \mathrm{O} & -1.141801 & 5.756664 & -0.840098 \\ \mathrm{O} & -3.545260 & 5.590069 & -0.974191\end{array}$

SW Defected Nanotube-2O (Triplet) $\mathrm{ZPE}=1.189013 \quad \mathrm{E}=\mathbf{=}-5651.801772$

$\begin{array}{lrrr}\text { C } & -5.078673 & -5.595263 & -0.431208 \\ \text { C } & -5.420513 & -4.907614 & 1.897697 \\ \text { C } & -5.810287 & -3.089589 & 3.502181 \\ \text { C } & -6.158559 & -0.706725 & 3.984222 \\ \text { C } & -6.446198 & 1.616354 & 3.285319 \\ \text { C } & -5.273065 & 1.587633 & -4.485936 \\ \text { C } & -4.881397 & -4.933218 & -2.785109 \\ \text { C } & -4.281882 & -5.354029 & -1.575877 \\ \text { C } & -4.563122 & -5.325563 & 0.854351 \\ \text { C } & -4.945032 & -4.008084 & 2.885825 \\ \text { C } & -5.319150 & -1.837641 & 3.960778 \\ \text { C } & -5.632086 & 0.587048 & 3.866424 \\ \text { C } & -5.862978 & 2.715063 & 2.681318 \\ \text { C } & -5.756801 & 4.164210 & 0.646742 \\ \text { C } & -5.215228 & 4.136585 & -1.848949 \\ \text { C } & -4.763320 & 2.670410 & -3.729653 \\ \text { C } & -4.457139 & 0.514262 & -4.827045 \\ \text { C } & -4.252046 & -1.928051 & -4.829199 \\ \text { C } & -4.177354 & -4.084563 & -3.664841 \\ \text { C } & -2.862236 & -5.206008 & -1.414846 \\ \text { C } & -3.140518 & -5.158386 & 1.011404 \\ \text { C } & -3.522001 & -3.825712 & 3.028143 \\ \text { C } & -3.905836 & -1.647306 & 4.084429 \\ \text { C } & -4.214331 & 0.780569 & 4.005422 \\ \text { C } & -4.451273 & 2.936965 & 2.939105 \\ \text { C } & -4.421569 & 4.516465 & 1.056686 \\ \text { C } & -3.827879 & 4.511352 & -1.857141 \\ \text { C } & -3.354850 & 2.852708 & -3.555296 \\ \text { C } & -3.039528 & 0.659851 & -4.634721 \\ \text { C } & -2.826414 & -1.787619 & -4.657163\end{array}$




\begin{tabular}{|c|c|c|c|}
\hline C & -2.755630 & -3.946663 & -3.50491 \\
\hline C & -2.086205 & -4.646102 & -2.46665 \\
\hline C & -2.283639 & -5.274333 & -0.11324 \\
\hline $\mathrm{C}$ & -2.625917 & -4.549174 & 2.1924 \\
\hline $\mathrm{C}$ & -3.021998 & -2.717894 & $3.7576^{\prime}$ \\
\hline $\mathrm{C}$ & -3.374425 & -0.336604 & 4.22352 \\
\hline C & -3.622443 & 2.028271 & 3.6282 \\
\hline C & -3.811649 & 3.933664 & 2.2285 \\
\hline $\mathrm{C}$ & -3.443351 & 5.137908 & 0.351 \\
\hline C & -2.852520 & 3.891686 & -2.6724 \\
\hline $\mathrm{C}$ & -2.490520 & 1.869219 & -4.138 \\
\hline $\mathrm{C}$ & -2.218892 & -0.503748 & -4.7217 \\
\hline $\mathrm{C}$ & -2.071304 & -2.873668 & -4.1489 \\
\hline $\mathrm{C}$ & -0.671445 & -4.506643 & -2.3110 \\
\hline $\mathrm{C}$ & -0.871840 & -5.117812 & 0.046 \\
\hline $\mathrm{C}$ & -1.221367 & -4.379010 & 2.347 \\
\hline $\mathrm{C}$ & -1.619009 & -2.532514 & 3.8955 \\
\hline C & -1.960203 & -0.137640 & 4.3169 \\
\hline $\mathrm{C}$ & -2.191968 & 2.232518 & 3.655 \\
\hline $\mathrm{C}$ & -2.428755 & 4.163171 & 2.243 \\
\hline $\mathrm{C}$ & -1.020539 & 5.217139 & 0.4552 \\
\hline $\mathrm{C}$ & -1.358531 & 4.063622 & -2.64 \\
\hline $\mathrm{C}$ & -1.065502 & 2.003998 & -4.0451 \\
\hline $\mathrm{C}$ & -0.813208 & -0.378121 & -4.5949 \\
\hline $\mathrm{C}$ & -0.660919 & -2.746133 & -4.0067 \\
\hline $\mathrm{C}$ & 0.059909 & -3.680409 & -3.2051 \\
\hline $\mathrm{C}$ & -0.045521 & -4.906241 & -1.09 \\
\hline $\mathrm{C}$ & -0.330515 & -4.828021 & 1.3274 \\
\hline $\mathrm{C}$ & -0.722211 & -3.470057 & 3.321 \\
\hline C & -1.106550 & -1.268661 & 4.31 \\
\hline $\mathrm{C}$ & -1.389253 & 1.160215 & 4.144 \\
\hline $\mathrm{C}$ & -1.547550 & 3.347855 & 2.985 \\
\hline $\mathrm{C}$ & -2.189935 & 4.986578 & 1.062 \\
\hline $\mathrm{C}$ & -0.635908 & 4.886540 & -1.7818 \\
\hline $\mathrm{C}$ & -0.504572 & 3.150734 & -3.40 \\
\hline $\mathrm{C}$ & -0.229593 & 0.910537 & -4.4065 \\
\hline $\mathrm{C}$ & -0.018746 & -1.539859 & -4.3873 \\
\hline $\mathrm{C}$ & 1.468396 & -3.547072 & -3.0608 \\
\hline C & 1.361227 & -4.750245 & -0.9380 \\
\hline $\mathrm{C}$ & 1.077197 & -4.654547 & 1.4859 \\
\hline $\mathrm{C}$ & 0.683970 & -3.292200 & 3.4758 \\
\hline $\mathrm{C}$ & 0.291581 & -1.090662 & 4.46 \\
\hline $\mathrm{C}$ & 0.014207 & 1.338768 & 4.2606 \\
\hline $\mathrm{C}$ & -0.074647 & 3.477276 & 2.9892 \\
\hline $\mathrm{C}$ & 0.319422 & 4.911276 & 0.85 \\
\hline $\mathrm{C}$ & 0.744054 & 4.810677 & -1.5652 \\
\hline $\mathrm{C}$ & 0.929600 & 3.247852 & -3.37 \\
\hline
\end{tabular}




\begin{tabular}{|c|c|c|c|}
\hline $\mathrm{C}$ & 1.195051 & 1.019864 & -4.350575 \\
\hline $\mathrm{C}$ & 1.397305 & -1.424977 & -4.27706 \\
\hline $\mathrm{C}$ & 2.154856 & -2.503358 & -3.74763 \\
\hline C & 2.140749 & -4.220091 & -2.00466 \\
\hline C & 1.940139 & -4.786580 & 0.36476 \\
\hline $\mathrm{C}$ & 1.588488 & -4.024221 & 2.65840 \\
\hline C & 1.177497 & -2.175011 & 4.1991 \\
\hline C & 0.826709 & 0.217626 & 4.588 \\
\hline C & 0.641833 & 2.553332 & 3.81115 \\
\hline $\mathrm{C}$ & 0.742463 & 4.320490 & 2.09857 \\
\hline C & 1.233573 & 5.044125 & -0.22350 \\
\hline $\mathrm{C}$ & 1.560436 & 4.140428 & -2.46822 \\
\hline C & 1.778243 & 2.240739 & -3.94627 \\
\hline $\mathrm{C}$ & 2.012494 & -0.152272 & -4.421 \\
\hline $\mathrm{C}$ & 3.568927 & -2.384764 & -3.62468 \\
\hline C & 3.553159 & -4.070349 & -1.85174 \\
\hline C & 3.349032 & -4.609142 & 0.52 \\
\hline C & 2.992883 & -3.839540 & 2.8152 \\
\hline $\mathrm{C}$ & 2.578762 & -2.004040 & 4.37105 \\
\hline C & 2.227061 & 0.374421 & 4.80696 \\
\hline C & 2.052874 & 2.699953 & 4.0637 \\
\hline $\mathrm{C}$ & 2.175157 & 4.394413 & 2.32 \\
\hline C & 2.614744 & 4.993357 & -0.0041 \\
\hline $\mathrm{C}$ & 2.975796 & 4.184074 & -2.27519 \\
\hline C & 3.211022 & 2.344016 & -3.8455 \\
\hline $\mathrm{C}$ & 3.429605 & -0.045301 & -4.3354 \\
\hline C & 4.216190 & -1.205705 & $-4.0770 s$ \\
\hline C & 4.285069 & -3.285551 & -2.7829 \\
\hline C & 4.174017 & -4.425544 & -0.62213 \\
\hline $\mathrm{C}$ & 3.884096 & -4.292296 & 1.8018 \\
\hline C & 3.482456 & -2.936475 & 3.7995 \\
\hline C & 3.078356 & -0.758766 & 4.8461 \\
\hline C & 2.795419 & 1.668029 & 4.7137 \\
\hline C & 2.775631 & 3.737488 & 3.43804 \\
\hline C & 3.075873 & 4.879032 & 1.3232 \\
\hline C & 3.503458 & 4.763278 & -1.0957 \\
\hline C & 3.811369 & 3.405097 & -3.1006 \\
\hline $\mathrm{C}$ & 4.031042 & 1.237457 & -4.18524 \\
\hline C & 5.648621 & -1.099155 & -3.97442 \\
\hline $\mathrm{C}$ & 5.709658 & -3.156349 & $-2.6454^{\circ}$ \\
\hline C & 5.593449 & -4.261937 & -0.4636 \\
\hline C & 5.302622 & -4.107217 & 1.9637 \\
\hline $\mathrm{C}$ & 4.901415 & -2.755587 & 3.97173 \\
\hline $\mathrm{C}$ & 4.489781 & -0.595445 & 5.0643 \\
\hline C & 4.194857 & 1.834749 & 4.99245 \\
\hline C & 4.183550 & 3.906743 & 3.7138 \\
\hline C & 4.498714 & 4.970388 & 1.54908 \\
\hline
\end{tabular}




\begin{tabular}{|c|c|c|c|}
\hline $\mathrm{C}$ & 4.916676 & 4.794064 & -0.872372 \\
\hline $\mathrm{C}$ & 5.235194 & 3.481308 & -2.922027 \\
\hline $\mathrm{C}$ & 5.467687 & 1.341000 & -4.076019 \\
\hline C & 6.351718 & -3.789529 & -1.559182 \\
\hline C & 6.141616 & -4.269847 & 0.840488 \\
\hline $\mathrm{C}$ & 5.782370 & -3.482743 & 3.140496 \\
\hline C & 5.358583 & -1.669466 & 4.751729 \\
\hline C & 4.983081 & 0.702628 & 5.304728 \\
\hline C & 4.814111 & 3.049312 & 4.633352 \\
\hline $\mathrm{C}$ & 4.984668 & 4.669561 & 2.833936 \\
\hline $\mathrm{C}$ & 5.374077 & 5.077612 & 0.441745 \\
\hline C & 5.761483 & 4.286077 & -1.882027 \\
\hline C & 6.043142 & 2.527567 & -3.580876 \\
\hline $\mathrm{H}$ & -6.349594 & 1.494851 & -4.590583 \\
\hline $\mathrm{H}$ & -6.492150 & -5.035683 & 1.779457 \\
\hline $\mathrm{H}$ & -6.152185 & -5.702395 & -0.553897 \\
\hline $\mathrm{H}$ & -5.957091 & -5.030066 & -2.897298 \\
\hline $\mathrm{H}$ & -6.882663 & -3.217772 & 3.389832 \\
\hline $\mathrm{H}$ & -7.227214 & -0.848729 & 3.852675 \\
\hline $\mathrm{H}$ & -7.498826 & 1.402749 & 3.124987 \\
\hline $\mathrm{H}$ & 6.852492 & -3.340406 & 3.257686 \\
\hline $\mathrm{H}$ & 7.211368 & -4.125965 & 0.958974 \\
\hline $\mathrm{H}$ & 7.424116 & -3.667196 & -1.440577 \\
\hline $\mathrm{H}$ & 7.121610 & 2.579273 & -3.466720 \\
\hline $\mathrm{H}$ & 6.427116 & -1.535505 & 4.891037 \\
\hline $\mathrm{H}$ & 6.836867 & 4.305007 & -1.732641 \\
\hline $\mathrm{H}$ & 6.046071 & 0.840293 & 5.477975 \\
\hline $\mathrm{H}$ & 6.442682 & 5.122682 & 0.628357 \\
\hline $\mathrm{H}$ & 5.874349 & 3.177898 & 4.828052 \\
\hline $\mathrm{H}$ & 6.046930 & 4.759587 & 3.038301 \\
\hline C & -6.509125 & 3.433883 & 1.601581 \\
\hline $\mathrm{H}$ & -7.536948 & 3.168045 & 1.371863 \\
\hline C & -6.129993 & 4.267668 & -0.706838 \\
\hline $\mathrm{H}$ & -7.164673 & 4.031265 & -0.944691 \\
\hline $\mathrm{C}$ & -5.670392 & 3.359640 & -2.899197 \\
\hline $\mathrm{H}$ & -6.724618 & 3.110148 & -2.965110 \\
\hline $\mathrm{C}$ & -5.010505 & -0.771781 & -5.081640 \\
\hline $\mathrm{H}$ & -6.084184 & -0.857938 & -5.217180 \\
\hline $\mathrm{C}$ & -4.866191 & -3.150166 & -4.476687 \\
\hline $\mathrm{H}$ & -5.940429 & -3.251668 & -4.597606 \\
\hline $\mathrm{C}$ & 6.249630 & 0.165220 & -4.153458 \\
\hline $\mathrm{H}$ & 7.328420 & 0.250407 & -4.064995 \\
\hline $\mathrm{C}$ & 6.377362 & -2.161193 & -3.398044 \\
\hline $\mathrm{H}$ & 7.454253 & -2.063652 & -3.299193 \\
\hline 0 & -1.175811 & 5.759548 & -0.829809 \\
\hline 0 & -3.577055 & 5.579930 & -0.965029 \\
\hline
\end{tabular}


SW Defected Nanotube-2O (Quintet) $\mathrm{ZPE}=1.189557 \quad \mathrm{Ee}=\quad-5651.796547$

\begin{tabular}{|c|c|c|c|}
\hline $\mathrm{C}$ & -5.084203 & -5.568987 & -0.434818 \\
\hline $\mathrm{C}$ & -5.430568 & -4.905994 & 1.901704 \\
\hline $\mathrm{C}$ & -5.819802 & -3.091138 & 3.508124 \\
\hline $\mathrm{C}$ & -6.167154 & -0.708625 & 3.989909 \\
\hline $\mathrm{C}$ & -6.452248 & 1.613262 & 3.291232 \\
\hline $\mathrm{C}$ & -5.264154 & 1.594927 & -4.500625 \\
\hline $\mathrm{C}$ & -4.881266 & -4.906588 & -2.790172 \\
\hline $\mathrm{C}$ & -4.281711 & -5.339969 & -1.585370 \\
\hline $\mathrm{C}$ & -4.569377 & -5.318426 & 0.839287 \\
\hline $\mathrm{C}$ & -4.955167 & -4.008503 & 2.877276 \\
\hline $\mathrm{C}$ & -5.327512 & -1.838397 & 3.961002 \\
\hline $\mathrm{C}$ & -5.639750 & 0.589310 & 3.865199 \\
\hline $\mathrm{C}$ & -5.866399 & 2.717534 & 2.676222 \\
\hline $\mathrm{C}$ & -5.752516 & 4.160550 & 0.641372 \\
\hline $\mathrm{C}$ & -5.209225 & 4.132099 & -1.850268 \\
\hline $\mathrm{C}$ & -4.755955 & 2.674931 & -3.736801 \\
\hline $\mathrm{C}$ & -4.448625 & 0.522636 & -4.842178 \\
\hline $\mathrm{C}$ & -4.246822 & -1.922681 & -4.842602 \\
\hline $\mathrm{C}$ & -4.174464 & -4.076456 & -3.671451 \\
\hline $\mathrm{C}$ & -2.867459 & -5.201409 & -1.424538 \\
\hline $\mathrm{C}$ & -3.146020 & -5.154617 & 0.999831 \\
\hline $\mathrm{C}$ & -3.527618 & -3.822564 & 3.018434 \\
\hline $\mathrm{C}$ & -3.912884 & -1.646866 & 4.080481 \\
\hline $\mathrm{C}$ & -4.219248 & 0.780417 & 4.002758 \\
\hline $\mathrm{C}$ & -4.454098 & 2.937560 & 2.937304 \\
\hline $\mathrm{C}$ & -4.420493 & 4.515965 & 1.055009 \\
\hline $\mathrm{C}$ & -3.823358 & 4.510412 & -1.857112 \\
\hline C & -3.348014 & 2.855844 & -3.559254 \\
\hline $\mathrm{C}$ & -3.030785 & 0.666286 & -4.643113 \\
\hline $\mathrm{C}$ & -2.818162 & -1.781582 & -4.664093 \\
\hline $\mathrm{C}$ & -2.753421 & -3.941564 & -3.511574 \\
\hline $\mathrm{C}$ & -2.087764 & -4.638059 & -2.474868 \\
\hline C & -2.289843 & -5.268160 & -0.120071 \\
\hline $\mathrm{C}$ & -2.631534 & -4.549182 & 2.186604 \\
\hline $\mathrm{C}$ & -3.029232 & -2.719610 & 3.749588 \\
\hline $\mathrm{C}$ & -3.381646 & -0.338789 & 4.218177 \\
\hline $\mathrm{C}$ & -3.626677 & 2.027501 & 3.627040 \\
\hline C & -3.812840 & 3.934605 & 2.228725 \\
\hline $\mathrm{C}$ & -3.439618 & 5.135804 & 0.350338 \\
\hline $\mathrm{C}$ & -2.847217 & 3.891213 & -2.672959 \\
\hline $\mathrm{C}$ & -2.483102 & 1.872166 & -4.142922 \\
\hline $\mathrm{C}$ & -2.210664 & -0.499674 & -4.729470 \\
\hline $\mathrm{C}$ & -2.065211 & -2.867803 & -4.158611 \\
\hline $\mathrm{C}$ & -0.670434 & -4.500496 & -2.316375 \\
\hline $\mathrm{C}$ & -0.875306 & -5.113119 & 0.041379 \\
\hline
\end{tabular}




\begin{tabular}{|c|c|c|c|}
\hline C & -1.229349 & -4.380647 & 2.34221 \\
\hline C & -1.625732 & -2.535511 & 3.8893 \\
\hline C & -1.965033 & -0.140565 & 4.31299 \\
\hline $\mathrm{C}$ & -2.195971 & 2.230872 & $3.6554 c$ \\
\hline $\mathrm{C}$ & -2.429713 & 4.163319 & 2.2450 \\
\hline $\mathrm{C}$ & -1.018869 & 5.213317 & 0.45776 \\
\hline C & -1.352779 & 4.062644 & $-2.6401\}$ \\
\hline C & -1.056800 & 2.006401 & -4.04582 \\
\hline $\mathrm{C}$ & -0.805166 & -0.374987 & -4.598 \\
\hline C & -0.656473 & -2.742184 & -4.013 \\
\hline C & 0.062157 & -3.677305 & -3.207 \\
\hline $\mathrm{C}$ & -0.046527 & -4.904329 & -1.101 \\
\hline $\mathrm{C}$ & -0.336493 & -4.827311 & 1.320 \\
\hline $\mathrm{C}$ & -0.730536 & -3.470573 & 3.3153 \\
\hline $\mathrm{C}$ & -1.112822 & -1.271234 & 4.315 \\
\hline $\mathrm{C}$ & -1.394099 & 1.156225 & 4.14 \\
\hline $\mathrm{C}$ & -1.550820 & 3.344207 & 2.986 \\
\hline $\mathrm{C}$ & -2.188302 & 4.983640 & 1.06 \\
\hline $\mathrm{C}$ & -0.632571 & 4.884607 & -1.7795 \\
\hline $\mathrm{C}$ & -0.497242 & 3.151025 & -3.4081 \\
\hline $\mathrm{C}$ & -0.220914 & 0.914195 & -4.4082 \\
\hline $\mathrm{C}$ & -0.012659 & -1.535821 & -4.3 \\
\hline $\mathrm{C}$ & 1.471657 & -3.544752 & -3.060 \\
\hline $\mathrm{C}$ & 1.358602 & -4.750262 & -0.9411 \\
\hline $\mathrm{C}$ & 1.072567 & -4.655113 & 1.4812 \\
\hline $\mathrm{C}$ & 0.678472 & -3.293624 & 3.472 \\
\hline $\mathrm{C}$ & 0.284509 & -1.094414 & 4.46 \\
\hline $\mathrm{C}$ & 0.008957 & 1.333843 & 4.2620 \\
\hline $\mathrm{C}$ & -0.076821 & 3.472438 & 2.9911 \\
\hline C & 0.318214 & 4.906817 & 0.8 \\
\hline $\mathrm{C}$ & 0.747994 & 4.809566 & -1.5612 \\
\hline $\mathrm{C}$ & 0.936840 & 3.248914 & -3.371 \\
\hline $\mathrm{C}$ & 1.203180 & 1.022819 & -4.3490 \\
\hline $\mathrm{C}$ & 1.404960 & -1.421566 & -4.2760 \\
\hline $\mathrm{C}$ & 2.160739 & -2.501155 & -3.7477 \\
\hline $\mathrm{C}$ & 2.140568 & -4.217715 & -2.0058 \\
\hline $\mathrm{C}$ & 1.935367 & -4.786077 & 0.3634 \\
\hline $\mathrm{C}$ & 1.583127 & -4.027675 & 2.6573 \\
\hline C & 1.170939 & -2.180322 & 4.1973 \\
\hline $\mathrm{C}$ & 0.820138 & 0.212382 & 4.587 \\
\hline $\mathrm{C}$ & 0.637791 & 2.549326 & 3.8135 \\
\hline $\mathrm{C}$ & 0.740705 & 4.316651 & 2.102 \\
\hline C & 1.234192 & 5.039695 & -0.2189 \\
\hline $\mathrm{C}$ & 1.566048 & 4.141862 & -2.4637 \\
\hline $\mathrm{C}$ & 1.785796 & 2.242427 & -3.941 \\
\hline $\mathrm{C}$ & 2.020521 & -0.150361 & -4.4186 \\
\hline $\mathrm{C}$ & 3.573811 & -2.383608 & -3.622 \\
\hline
\end{tabular}




\begin{tabular}{|c|c|c|c|}
\hline $\mathrm{C}$ & 3.554543 & -4.069613 & -1.85046 \\
\hline $\mathrm{C}$ & 3.346223 & -4.610172 & 0.52502 \\
\hline $\mathrm{C}$ & 2.986250 & -3.844429 & 2.81616 \\
\hline $\mathrm{C}$ & 2.572358 & -2.009962 & 4.3709 \\
\hline $\mathrm{C}$ & 2.222445 & 0.368652 & 4.8077 \\
\hline $\mathrm{C}$ & 2.048877 & 2.695171 & 4.0673 \\
\hline $\mathrm{C}$ & 2.172682 & 4.391013 & 2.3310 \\
\hline $\mathrm{C}$ & 2.615845 & 4.990576 & 0.0028 \\
\hline $\mathrm{C}$ & 2.981037 & 4.185413 & -2.2685 \\
\hline $\mathrm{C}$ & 3.219458 & 2.345369 & -3.8 \\
\hline $\mathrm{C}$ & 3.437792 & -0.043843 & -4.3296 \\
\hline $\mathrm{C}$ & 4.222608 & -1.203883 & -4.0 \\
\hline $\mathrm{C}$ & 4.287927 & -3.285909 & -2.77 \\
\hline $\mathrm{C}$ & 4.173426 & -4.428578 & -0.62 \\
\hline $\mathrm{C}$ & 3.879148 & -4.296281 & 1.8 \\
\hline $\mathrm{C}$ & 3.475082 & -2.940582 & 3.75 \\
\hline $\mathrm{C}$ & 3.072076 & -0.763962 & 4.84 \\
\hline C & 2.790532 & 1.661938 & 4.71 \\
\hline C & 2.772075 & 3.732052 & 3.44 \\
\hline C & 3.074735 & 4.876387 & 1.3 \\
\hline $\mathrm{C}$ & 3.506260 & 4.763002 & -1.08 \\
\hline $\mathrm{C}$ & 3.818106 & 3.406141 & -3.09 \\
\hline $\mathrm{C}$ & 4.039523 & 1.239370 & -4.17 \\
\hline $\mathrm{C}$ & 5.655988 & -1.098138 & -3.965 \\
\hline $\mathrm{C}$ & 5.712892 & -3.157576 & -2.63 \\
\hline $\mathrm{C}$ & 5.591548 & -4.266112 & -0.45 \\
\hline $\mathrm{C}$ & 5.298318 & -4.112819 & 1.96 \\
\hline $\mathrm{C}$ & 4.895929 & -2.761115 & 3.97 \\
\hline $\mathrm{C}$ & 4.483029 & -0.601569 & 5.06 \\
\hline $\mathrm{C}$ & 4.189527 & 1.827908 & 4.9 \\
\hline $\mathrm{C}$ & 4.180551 & 3.901296 & 3.72 \\
\hline $\mathrm{C}$ & 4.497329 & 4.968382 & 1.55 \\
\hline $\mathrm{C}$ & 4.919392 & 4.794318 & -0.86 \\
\hline $\mathrm{C}$ & 5.241683 & 3.482102 & -2.91 \\
\hline $\mathrm{C}$ & 5.475790 & 1.342205 & -4.0 \\
\hline $\mathrm{C}$ & 6.352129 & -3.790787 & -1.5530 \\
\hline $\mathrm{C}$ & 6.137846 & -4.273713 & 0.84 \\
\hline $\mathrm{C}$ & 5.777004 & -3.490975 & 3.1 \\
\hline $\mathrm{C}$ & 5.352174 & -1.677180 & 4.7557 \\
\hline $\mathrm{C}$ & 4.977215 & 0.695393 & 5.30 \\
\hline $\mathrm{C}$ & 4.809723 & 3.043381 & 4.6397 \\
\hline $\mathrm{C}$ & 4.981823 & 4.667393 & 2.84 \\
\hline $\mathrm{C}$ & 5.374268 & 5.076601 & 0.4529 \\
\hline $\mathrm{C}$ & 5.766062 & 4.287100 & -1.8704 \\
\hline $\mathrm{C}$ & 6.050533 & 2.528061 & -3.5 \\
\hline $\mathrm{H}$ & -6.340385 & 1.504122 & -4.61 \\
\hline $\mathrm{H}$ & -6 & 1230 & 1 \\
\hline
\end{tabular}




$\begin{array}{rrrr}\mathrm{H} & -6.158599 & -5.662459 & -0.561060 \\ \mathrm{H} & -5.958611 & -4.991285 & -2.896591 \\ \mathrm{H} & -6.892648 & -3.223119 & 3.405107 \\ \mathrm{H} & -7.236898 & -0.849823 & 3.866184 \\ \mathrm{H} & -7.506806 & 1.404710 & 3.136961 \\ \mathrm{H} & 6.847180 & -3.351551 & 3.266511 \\ \mathrm{H} & 7.207454 & -4.129650 & 0.965458 \\ \mathrm{H} & 7.424240 & -3.668464 & -1.431721 \\ \mathrm{H} & 7.128751 & 2.579308 & -3.451701 \\ \mathrm{H} & 6.420516 & -1.543690 & 4.896937 \\ \mathrm{H} & 6.841176 & 4.306524 & -1.719165 \\ \mathrm{H} & 6.040430 & 0.832284 & 5.479642 \\ \mathrm{H} & 6.442559 & 5.121674 & 0.641413 \\ \mathrm{H} & 5.869759 & 3.171501 & 4.835863 \\ \mathrm{H} & 6.043767 & 4.758166 & 3.049756 \\ \mathrm{C} & -6.508959 & 3.427043 & 1.606300 \\ \mathrm{H} & -7.538980 & 3.168020 & 1.378524 \\ \mathrm{C} & -6.121839 & 4.245033 & -0.705687 \\ \mathrm{H} & -7.152500 & 3.990786 & -0.943312 \\ \mathrm{C} & -5.662992 & 3.358728 & -2.904759 \\ \mathrm{H} & -6.716981 & 3.108353 & -2.971832 \\ \mathrm{C} & -5.001604 & -0.761184 & -5.107968 \\ \mathrm{H} & -6.073819 & -0.846005 & -5.254882 \\ \mathrm{C} & -4.861373 & -3.136774 & -4.496402 \\ \mathrm{H} & -5.934809 & -3.240227 & -4.622260 \\ \mathrm{C} & 6.257561 & 0.165727 & -4.142259 \\ \mathrm{H} & 7.336215 & 0.250561 & -4.051952 \\ \mathrm{C} & 6.382913 & -2.161761 & -3.390640 \\ \mathrm{H} & 7.459832 & -2.065885 & -3.290644 \\ \mathrm{O} & -1.173774 & 5.753865 & -0.826785 \\ \mathrm{O} & -3.574444 & 5.581295 & -0.965453 \\ & & & \end{array}$

